\title{
SET IN PERCEPTION
}

\author{
A dissertation presented to \\ the Victoria University of Wellington \\ in fulfilment of the requirements \\ for the degree of
}

Doctor of Philosophy by John Alasdair Gribben, M.A. 


\section{ACKNOWLEDGMENT}

The author is grateful for the co-operation of Harry Clarkson and Graham Goodger for their design and manufacture of apparatus; to the University Mechanics and Carpenters' Workshops; to Ken White for assisting to adjust apparatus; to the University Photographer and Central Document Reproduction Service for photographs; and to subjects, who were unpaid. 
CONTENTS

Page

Tables

Figures

iv

INTRODUCTION

Theory and early experiments. 1

Recent experiments on the effect of instructions. 7

Cognitive factors in experiments on set. 12

The effect of number of alternatives. 16

Stimulus identification experiments. 19

$\begin{array}{ll}\text { Summary remarks. } & 23\end{array}$

GENERAL METHOD 25

Apparatus. $\quad 26$

Stimuli. $\quad 29$

Alternatives. 31

Response recording. 35

Subjects. $\quad 35$

General procedure. $\quad 35$

Experimental design. $\quad 37$

Discussion of variables used in the present study 39

\begin{tabular}{l|l} 
EXPERIMENT I & 43
\end{tabular}

EXPERIMENT II $\quad 55$

COMPARISON OF EXPERIMENTS I AND II 63

$\begin{array}{lll}\text { EXPERIMENT III } & 68\end{array}$

$\begin{array}{ll}\text { EXPERIMENT IV } & 75\end{array}$

$\begin{array}{ll}\text { EXPERIMENT V } & 89\end{array}$

EXPERIMENT VI $\quad 102$

$\begin{array}{lll}\text { SUMMARY OF RESULTS } & 110\end{array}$

$\begin{array}{lr}\text { DISCUSSION } & 114\end{array}$ 
DISCUSSION ( continued)

Numerical stimulus uncertainty. 122

Stimulus discriminability. 125

The preparation effect.

SUMMARY

APPENDIX

1. Apparatus.

2. Experiment I.

3. Experiment II. 
1. Basic Plan of Experiments.

2. Experiment I: Analysis of Variance.

3. Experiment I: Analysis of Covariance.

4. Experiment II: Analysis of Variance.

5. Experiments I and II: Analysis of Variance.

6. Experiments I and II: Cell mean totals.

7. Experiment III: Analysis of Variance.

8. Experiment III: Accuracy totals.

9. Experiment IV: Analysis of Variance, First Choices.

10. Experiment IV: Analysis of Variance, Ranked Choices.

11. Experiment IV: Analysis of Variance, First and Second Choices.

12. Experiment IV: Comparison of F Ratios for

$$
\text { the different scoring systems. }
$$

13. Experiment V: Analysis of Variance,

First Choices.

14. Experiment V: Analysis of Variance, Ranked Choices.

15. Experiment V: Analysis of Variance, First and Second Choices.

16. Experiment $\mathrm{V}$ : Comparison of $\mathrm{F}$ ratios for the different scoring systems.

17. Experiments IV and V: Proportions of First Choices Correct. 
18. Experiment V: Analysis of Variance,

$$
\text { stimulus Series. }
$$

19. Experiment VI: Analysis of Variance.

105

20. Summary of major results.

21. Experiment II: Analysis of Variance. 
1. Apparatus.

2. Apparatus. 28

3. Examples of stimuli. 30

4. Similar Alternatives for Shapes. 32

5. Dissimilar Alternatives for Multiple Figures. 33

6. Examples of Similar and Dissimilar Alternatives.34

7. I: Sequence x Exposure. 48

8. I: Exposure x Similarity. 48

9. II: Sequence x Stimulus. 59

10. I and II: Sequence $x$ Experiments $x$ Stimulus. 67

11. I and II: Sequence $x$ Exposure $x$ Similarity. 68

12. IV: Exposure x Similarity. 86

13. IV: Exposure $x$ Interval x Stimulus. 86

14. V: Exposure $x$ Interval x Stimulus x Similarity. 98

15. VI: Sequence x Similarity. 106

16. VI: Exposure $x$ Interval 106

17. Field Conditions. 133 
When a person is set, he is said to be prepared for a narrowed range of possible events. Instead of being equally prepared for all possible contingencies, he expects only a few. The general notion has been variously expressed as selective attention, specific expectancies or hypotheses, relative sensitisation, abstraction, perceptual bias, and in many other ways. Set, as a result of such preparation, is said to lead to greater efficiency of perception, and to greater efficiency of any later behaviour dependent upon the perception.

Reduced range of expectation has been the central point of study for most experimenters. There are, however, at least five main theories on the locus of set, although at times the theories are not distinguishable. If set narrows the range of expected events, increased efficiency of perception, or perception-dependent behaviour, can refer to five types of "preparation": facilitation of information handling processes, receptor adjustment, sensitisation of perception, memory processes or response processes. Other verbally distinct hypotheses about set are frequently invoked for particular types of perceptual activity, and where relevant to the present study, will be discussed later.

\section{THEORY AND EARLY EXPERIMENTS}

In 1904 Kulpe published an experiment on abstraction that W.L.Bryan had carried out in Kulpe's laboratory. Subjects were instructed to report on one aspect of a briefly exposed complex stimulus (four nonsense syllables in varying configuration, differently coloured). After the stimulus exposure, questions were asked about all stimulus aspects, and more accurate reports were obtained 
on aspects corresponding to instructions than on incidental aspects. The instructions induced an Aufgabe or set (task attitude) which created a predisposition for a particular stimulus aspect, altering the apprehension of the whole stimulus and eventual accuracy of report. The effect of instructions on accuracy was an "abstraction in the sense of an accentuation" of the stimulus aspect required for report. Earlier, Kulpe (1893, see Humphrey, 1951) had pointed out that reaction times differ according to the preparation of the subject. This result was found to be equivocal (Woodworth, 1938), but the suggestion that preparation may alter an apparently simple $S-R$ sequence stimulated the Wurzburgers to develop the important notion that "mental activities" can be directed by the subject's acceptance of a task.

Kulpe and Bryan asked their subjects four questions (number, letters, colours, configuration) after the stimulus exposure, with the question corresponding to the task always given first. Rubin (1913, see Wilcocks, 1925) pointed out that Kulpe's conclusions about the abstraction effect, because of the temporal order of questions and forgetting, were "immeasurably discredited" (1913, p.386). Kulpe on his part was equally emphatic that forgetting, and "verbal deficiencies", did not occur (1904, p.67). Many writers were reluctant to dismiss Kulpe's conclusions, since there were other abstraction experiments, related more to concept formation, which pointed to the same conclusions. Humphrey (1951), in a full account of Wurzburg work, omits reference to Rubin's criticism, but notes the experiment was "repeated" by Chapman (1932, in which forgetting is not as crucial). The complete confounding of set or abstraction with forgetting in Kulpe and Bryan's experiment is interesting in the light of later theorising about set: Lawrence \& Coles (1954), Lawrence \& LaBerge (1956), Brown (1960), Sperling (1960), Harris \& Haber (1963), Haber (1964) 
and others specifically invoke forgetting in their discussions of the topic, and sometimes the effect of set (instructions) on temporal order of report (forgetting) has been studied as a major experimental intention: Kay \& Poulton (1951), Brown (1954), Broadbent (1957), Murdock (1963), and many others.

Wilcocks (1925) examined Kulpe's problem in some detail. Using complex stimuli very similar to those used in the earlier experiment, questions about the different aspects were given in counterbalanced order and results presented to show that questions corresponding to the task asked later ( 2 nd - 4th) are answered less accurately than noncorresponding questions asked first ( $53.77 \%$ vs. $59.37 \%$ correct respectively, an insignificant difference with the variable data), justifying Rubin's criticism. When corresponding and noncorresponding questions are summed over temporal order (1st - 4th), the effect of set is slight: $p \doteq .20$ from analysis of variance. Analysis of questions separately shows that set benefits reporting letters (to be reported in their approximate position within the total stimulus), but not other questions. Wilcocks also tested the effect of instructions with one question per stimulus exposure to avoid Rubin's criticism and other sequential effects, and the figures show that tasks corresponding to questions about colours and letters are performed better than when there is not task-question correspondence, but an analysis of variance yielded a significant tasks-within-questions effect only for colour questions. Instructions to observe colours resulted in improved accuracy when tested against accuracy of colour reports when observed under different, noncorresponding instructions.

However, this is a comparison between "positive abstraction" (instructions creating a "determination for 
a stimulus aspect") and "negative abstraction" (instructions creating a determination away from a stimulus aspect" on which report was required). While subjects soon come to expect all four questions on the complex stimulus, the comparison between the two groups of reports has rather limited generality. We might be able to conclude that abstraction processes alter "apprehension" (equivalent to "reportability", perhaps) of a stimulus field, as early writers did, but to test the possibility that specific instructions actually improve perception, or perceptual report, a more neutral comparison control is needed. Such a neutral condition was offerred by Kulpe, Wilcocks and, later, Chapman, in which subjects were given no special observational task, and the stimulus field was seen without an Aufgabe. In Wilcocks' data, accuracy of report under this condition, as might be expected, was between that of corresponding and noncorresponding tasks. There is statistical evidence in the data only for a positive abstraction effect for identifying letters (multiple questions) when tested against the accuracy of report after no special observational task was given. Although the subtotals in Wilcocks' data frequently suggest positive abstraction, the effect is not marked, and is statistically adequate for only one case out of seven possible tests. In all cases, however, unequal task difficulty, subject strategies and preferences of report prevent any convincing conclusion about the effect of instructions on accuracy in Wilcocks' experiments. There is no clear evidence for set.

This is not the case in Chapman's study (1932). Chapman presented irregularly arranged consonants as stimuli, indicating instructions for report in a glass box in front of the subject, either before or after the stimulus exposure. Only one report was required on any trial: either number, identity or location of the consonants, and instructions for response were given before or after the 
stimulus in a "haphazard" manner. For each type of report and for each subject, accuracy with instructions given before the stimulus was greater in nearly every case than for instructions given after the stimulus. A separate experimental series, where subjects reported on the same aspect continuously, on the whole gave results similar to those for prior instructions in the main experimental series. The data of the main series show less difference between pre- and post-exposure instructions for reporting on the number of consonants, which is the easiest type of report and to some extent involved in the other two types of report.

Chapman's results are more regular than those of Wilcocks. Chapman's exposures were shorter, an exposure adjustment was made for some subjects, and the stimulus itself was more homogeneous. His data furnish initially convincing evidence that instructions facilitate accuracy of report, evidence for set, preparation, abstraction or selective attention widely quoted in later surveys of these and related topics. Chapman was interested in phenomenological distinctions, and in discussing his results, identified "perceptual accentuation" and "subsequent surrogative processes" separately. Subjects' introspections showed Chapman that perceptual accentuation did occur, but efficiency of report when instructions are given before the stimulus may derive from "structuring of the phenomenal field" and "structural changes which have taken place in the surrogates intervening between the original field and report" (p.173), a possibility seriously countenanced by Lawrence \& Coles (1954), Brown (1960) and Sperling (1960). Chapman's conclusions after these speculations was that the report is based on "the principal aspects of the surrogative mass", and that the effect of an Aufgabe given before the stimulus exposure is, figuratively, "to provide a preliminary matrix to the 
structural values of which succeeding experience tends to conform" ( $p .173)$. An Aufgabe given after the stimulus exposure prevents restructuring of the surrogate along the lines essential for report with sufficient clarity to match accuracy for an Aufgabe given before the stimulus exposure.

In more recent terminology, we might call the "surrogative mass" the eventual storage from which responses are made, and the "preliminary matrix" would seem to refer to expectancies, schemas and the like. Distinguishing perception (the "phenomenal field") from immediate memory (the "surrogative mass") was for Chapman a conclusion from subjects' introspections; an objective distinction retaining usual definitions is rather more difficult to make, and the problem will be examined later.

Other early experiments on abstraction have used different techniques to show the selective nature of perception. Moore $(1905,1910)$ presented subjects successive rows of five figures with one figure in the series repeated in each row; subjects were to stop the series as soon as they noticed some figure twice, and introspect. Moore noted that perceiving the repeated figure tended to inhibit perception of other figures, and quantitative data do not contradict this observation. Grunbaum (1908, see Moore, 1910; Humphrey, 1951) showed his subjects two groups of figures for three seconds and told them to note the figure common to both groups; when subjects did, perception of other, unique figures was poorex.

These two and other experiments led up to concept formation experiments in which, it was said, abstraction of common perceptual elements from a stimulus series is generalised to form a concept class. 
Moore thought "mental categories" (generic perceptual characteristics, e.g. "round", "lines crossed at top") were involved when subjects were able to recognise the repeated pattern in his experiments. The immediate sensation is "assimilated"(Herbart, Wundt) to a mental category and compared with previous "ideas" of the repeated pattern. The eventual product of abstraction is a "concept" rather than an "image" or "feeling", and when Moore's subjects were asked to draw the patterns they had seen, they noted that their reproductions were sometimes influenced by associations (e.g. symmetrical curves became a chess piece, a circle with tails an omega). Bartlett (1916) observed the same phenomenon, and Carmichael, Hogan and Walter (1932) demonstrated the effect experimentally. Whatever else this cognitive influence on veridical report may mean, the problem is with us today as "coding processes" (Miller,1956; Pollack,1959; Harris \& Haber,1963; Lindley, 1963). Set, some experimenters maintain, facilitates coding of input, or assimilation of stimulus information into perceptual and/or cognitive categories, in preparation for response.

RECENT EXPERIMENTS ON THE EFFECT OF INSTRUCTIONS

In this section will be discussed experiments which employ complex stimuli and require more than one type of response from the subject. Such studies resemble the experiments of Kulpe, Wilcocks and Chapman.

Lawrence \& LaBerge (1956) showed two cards from the Wisconsin Card Sorting Test to subjects as stimuli, seen at 0.1 second exposure. Each of these cards has a number (one to four) of the same forms (either circles, triangles, crosses or stars) in the same colour (either yellow, green, red or blue). Groups of subjects were to record all 
dimensions either with equal emphasis or with one dimension emphasised; to record all dimensions with equal emphasis but with order of report either free or as specified by the experimenter; or to record either all dimensions with one emphasised or only one specified dimension. For one dimension or for an emphasised dimension, instructions were specifically given before the stimulus and subjects were asked to imagine dollar rewards for correct report.

A "Kulpe effect" (emphasis vs. equal) was observed $(p<.01)$, as was a marked difference between emphasised and non-emphasised dimension reports (positive vs. negative abstraction). Reporting only one dimension gave only slightly better scores than reporting an emphasised dimension (when other dimensions were recorded as well). Equal emphasis conditions yielded similar overall accuracy whether order of report was free or specified after the exposure by the experimenter. But when emphasis was equal and order of report specified by the experimenter after the exposure, first reports were more accurate than second reports $(p<.01)$ and second reports were more accurate than third reports $(p<.05)$ - the Kulpe effect can be duplicated just by the order in which responses are made, even though the $S$ s are set to given equal attention to all three stimulus dimensions" $(\mathrm{p} \cdot 16)$. The authors reason that if subjects instructed to give emphasis to one dimension have a strong tendency to report this dimension first, "this would mean that the selective effect of instructions operates primarily through memory and reponse factors... The transmitted information under tachistoscopic presentation tends to be constant irrespective of instructions" ( $\mathrm{p} .17)$; it is the manner of distribution of this information that instructions influence. Lawrence \& LaBerge's rationale is weakened by the fact that emphasis accuracy is a little higher than equal emphasis accuracy (both first reports), and by the rather greater difference 
between emphasis conditions than between order of report conditions. The equation of positive and negative abstraction with sequential forgetting is an interesting hypothesis, but it may be doubted whether Lawrence \& LaBerge's analysis has been sufficiently thorough. It does appear, however, that comparing first reports for emphasis and equal instructions is a fair comparison (although free order of report accuracy is not given), and the hypothesis that preparation improves perceptual report needs further examination.

Harris \& Haber (1963, Haber, 1964) used the same stimuli, general instructions and procedure as in Lawrence \& LaBerge's study to see if the effect of instructions operated via coding of the stimulus into dimensions (number, form, colour) or into "objects" (e.g. two blue triangles, three green circles). Subjects reported on dimensions. After training subjects to use assigned codes, scores were analysed to show significant effects for codes, instructions, order of report and codes $\mathrm{x}$ instructions-emphasised instruction accuracy exceeded unemphasised instruction accuracy only for dimensions coding. The authors state "Since the encoding process is so selective, it seems unnecessary to postulate that attention has a direct effect on perception"(p332). The authors do not comment on an unusual order of report accuracy for objects coding under emphasised instructions which tends to confirm a possibility that it is the decoding of the inefficient objects code which creates this interaction (which Haber may have had in mind in his replication study, 1964). There is also a suggestion that objects coding prevents forgetting (the whole stimulus must be "reviewed" for dimension reporting), but the analysis is carried out with two subject groups included who followed assigned codes incompletely, and lowerorder subject interactions are rather high. (In Haber's replication, codes $\mathrm{x}$ order of report is significant, and in 
both experiments forgetting is less marked for objects codes than for dimensions codes.) Left and right card scores were recorded separately, and the left-right main effect is significant. Also, in both experiments, emphasis instructions interacts with card position: there is little left-right difference when instructions emphasise report for one dimension.

The evidence that coding strategy affects accuracy of report appears to be acceptable, but along with the evidence the rehearsal theory of short term memory gets back-handed support. The authors' conclusion that attention need not have "a direct effect on perception" is perhaps not warranted. If, as seems likely, report was required on objects instead of dimensions and results for this type of report were to show analogous coding (and decoding) effects, we would be allowed to make the same conclusion. If this

were so, a more general hypothesis would be that coding and decoding would affect report according to report requirements. Coding strategy is still important, but the reorganisation processes it demands merely obscures the effect of attention.

That set or attention affects accuracy of report in these two experiments is shown by the left-right $\mathrm{x}$ instructions interaction (instructions altering the usual attention given to left sides of stimulus displays-a differential that practice without instructions does not eliminate (Harcum, Filion \& Dyer,1962), at least for predominantly left to right language readers; it is shown by the main effect of instructions and by emphasis instruction accuracy exceeding equal emphasis instruction accuracy when coding and decoding are suited to the task; and it is also shown by comparing first report accuracy for emphasis instructions and equal emphasis instructions ( $p<.01$, Haber, 1964, p.403). This last difference might 
be regarded as a rebuttal of Lawrence \& LaBerge's belief that the "selective effect of instructions operates primarily through memory and response factors." In Lawrence \& LaBerge's experiment and in Harris \& Haber's and Haber's experiments, the order of report effect (forgetting) is significant, but in these three experiments the selective effect of instructions is greater than the effect of order of report, and in Haber's experiment comparison of first reports shows a significant instructions or preparation effect when (sequential) forgetting is not relevant.

Brown (1960) tested the effect of a "selective process during perception" by giving auditory instructions either two seconds before or simultaneously with a visual presentation of eight digits or consonants. The stimuli were in two rows and four columns, two columns either side of a fixation point, and inner and outer columns were either consonants or digits and sometimes either red or black. Instructions for "letters" or "numbers" given two seconds before the stimulus exposure yielded greater accuracy of report than instructions given simultaneously with the stimulus exposure. Stimuli required for report could be located by columns. When stimulus classes could be located as well by column position and colour, the accuracy difference between the two instructions was even greater than when the required stimuli could be located only by class. When subjects were to report either "outer" or "inner", or "red" or "black" columns of stimuli, instructions before the stimulus exposure were not as good as simultaneous instructions (a minor difference). Although the design appears to preclude a forgetting explanation of the difference between before and simultaneous instructions, it does presuppose the auditory instructions take time to influence report (which is in fact shown for stimulus class reports), and does not prevent the possibility 
that simultaneous instructions interfere with perception of the stimuli. Brown recognised these features of his design, and goes on to say that only the first digit or consonant can be considered literally as an immediate report, and that the efficiency of report must therefore depend partly on efficiency of stimulus information storage. "The selective process concerns storage, i.e. learning, rather than perception" $(\mathrm{p} .180)$, and Brown supposes this notion is more economical in the absence of "contrary evidence".

It may be noted that early writers were quite sure that there was a selective effect in perception, while later experimenters are concerned to show that the effect of set on perception, when and if it exists, is only apparent: set influences coding, learning, forgetting or responding, but not perception. There is a real problem here, and to the extent that experimenters tease out black box specifications, they are real attempts to explain the possible effects of "preparation". However, it is doubtful whether experiments are successful. Response, and response preparation factors, apparently need more penetrating analysis than has been attempted. Experimenters can take theoretically neutral stands by describing their measures as "perceptual reports" and in the present introduction, this stand is taken whenever analysis of the $\mathrm{S}-\mathrm{R}$ sequence seems dubious.

COGNITIVE FACTORS IN EXPERIMENTS ON SET

In Siipola's study (1935) subjects were shown "ambiguous" words for approximately 0.1 second and gave responses according to induced sets. If "sael" was shown and subjects were expecting words of animals or birds, "seal" might be reported. Unfortunately, the experiment is susceptible to much the same social explanation usually 
made of McGinnies' "perceptual defence" (1949); pseudo-perceptual situations can demonstrate anything from attitudes to the Zeigarnik effect. As a distinctly perceptual problem, of course, this experiment of Siipola is not meaningless. Most cognitive, emotional and response factors can be regarded as independent of the basic perceptual process and some experiments appear to demonstrate the effect of expectation on perceptual report in the absence of confounding factors.

Postman \& Bruner (1949) instructed subjects to look for "food" and/or "colour" words in tachistoscopic presentations for threshold measurements, showing two words intersecting at $45^{\circ}$, one a "critical" word (food or colour) and one a "neutral" word. They found that when subjects were told that one of the words would be a colour word and their task would be to identify it ("single set"), thresholds for colour words were lower than when subjects were told that one of the words would be either a colour or a food word ("multiple set"). They noted less familiar colour words (indigo, azure) were not perceived so readily as familiar colour words (brown, green). Freeman \& Engler (1955), showing only one word per presentation, found critical words more easily seen than neutral words (that is, a "general" set), but there was only a minor and insignificant difference between single and multiple set. Familiarity (Thorndike-Lorge word count, 1944) interacted with set (unpublished studies): single set resulted in lower thresholds for familiar words but higher thresholds for unfamiliar words. Perhaps the differential ability to form specific expectancies lies behind these results.

Foster (1962) asked subjects to detect or identify a word in a group of nine ( $3 \times 3)$ when sets were either "functional" (animals, plants, etc.) or "structural" (initial letters specified), and either "broad, narrow or 
very narrow". Search time was measured by the subject pressing a hand switch; subjects detecting the word then located its position on a $3 \times 3$ panel of buttons, and subjects identifying the word told the experimenter what it was. The effect of broadness of set was significant only with detection for functional categories and with identification for structural categories (this latter an imposed discrimination effect: with more initial letters specified, search time was increased). With functional categories for detection under very narrow set, subjects monitored the specific word to be detected, and the difference between this set and the other two appears to contribute most to the significant F for broadness of set (a variable in a technical sense only). Foster suggests that if the broadness of set for functional categories were re-designed to reflect numbers of alternatives, the effect of differences in (functional) set may be more pronounced.

Reid, Henneman \& Long (1960) photographed 48 sports words out of focus and asked college students to identify the word from category descriptions on a card given to the subjects after the word had been presented. Set was manipulated by categorical restriction-"i.e. by giving $s$ more attributes of the category to which a stimulus-word belongs". Categories could be football or baseball, college or professional teams, names of teams or players, etc. The hypothesised effect of categorical restriction was found. Familiarisation with category members was also significant, but did not interact with category restriction. Half the subjects selected the stimulus word from category descriptions shown before the stimulus presentation as well as after it, but this type of preparation did not facilitate identification.

In these and other studies it is possible to make the generalisation that if subjects can construct stimulus 
classes to facilitate processing of stimulus input, inducing expectations may improve accuracy of report. This speculation implies that expectancies given to subjects should be real: uncertainty about the stimulus should be decreased from the subject's point of view. If the expectancy manipulated is the range of a functional category, we should ensure that range intervals are monotonic (Foster doubts this of her categories) and that subjects' understanding of category definition and membership is adequate for the experimental purpose. However, we are hypothesising about a unitary process and, what is more, in the absence of convincing evidence that cognitive sets can improve perceptual reports. Foster appears to think that expectancies are altered more importantly by number of possible alternatives rather than by cognitive processes, and in her introduction cites several experiments demonstrating this effect. In Reid, Henneman \& Long's study, categorical restriction is associated with reduction in number of alternatives, but the restriction alters accuracy whether or not subjects are prepared for it before the stimulus presentation.

Should experiments of the type reviewed here demonstrate cognitive facilitation, they may be showing nothing but a relation between accuracy and number of alternatives expected. If number of alternative stimuli presented or responses allowed show the same relation to report accuracy as cognitive facilitation, the simplifying assumption would be that subjects' expectancies can substitute for stimulus and/or response populations specified by the experiment. Cognition would then have a peripheral, rather than a coding, effect on expectation. This is again, of course, speculation.

Coding will be a determinant of response accuracy if subjects have not completed the necessary perceptual 
learning or analysis for a task. Seamen on watch, natural scientists in the field, airpilots in flight, artists studying their art, and so on, all "know what to look for". Adequate constructs of the target are obviously beneficial (Marx, Murphy \& Brownstein,1961; Baldwin, Wright \& Lehr, 1964), but when the target and the general perceptual situation are known, the importance of coding might be expected to diminish, and differentiable numbers of stimulus possibilities to become more likely determinants of accuracy.

THE EFFECT OF NUMBER OF ALTERNATIVES

It has been suggested above that the number of possible alternative stimuli (or responses) that the subject can expect affects accuracy of perceptual report. Most frequently, set is experimentally defined as a reduction in the number of stimuli the subject can expect to see. This experimental design, however, does not usually separate any differentiable effect of stimulus numbers from response numbers on the eventual report accuracy. Several experiments appear to have shown that it is response possibilities rather than stimulus possibilities which affect accuracy. Garner discusses the problem as "Stimulus uncertainty or response uncertainty?" (1962,p.2849). When the number of stimuli and responses are the same, accuracy of stimulus identification, or speeds of search or reaction, vary more or less regularly as the numbers of possible stimuli and responses are varied. As Garner says, the demonstration of this principle "will be rampant in the rest of this book" (1962, p.33).

Pollack (1959) varied response uncertainty independently of stimulus uncertainty by specifying possible responses, after stimuli. (spondees) had been presented in noise, with varying lists of words from which subjects chose their response. The subjects were well 
practised at the task and were thoroughly familiar with the 32-word message population. Pollack found that for each size of response class there was very little difference between 2, 4, 8, 16, 32 and also 64 word stimulus classes shown to subjects before the stimulus presentation. Stimulus classes were equal to or larger than response classes. For each stimulus class, accuracy increased as numbers of response alternatives decreased, and this relation "would remain unchanged" with corrections for chance accuracy. Pollack carried out other experiments as well, and his general conclusion was that the size of message source (the number of stimuli the subject could expect) is unimportant; accuracy of report is clearly a function of response uncertainty.

Long, Henneman \& Reid (1960) presented ambiguous letters to subjects to see if set affected sensitisation of perception or retention and response. Letters were "degraded" by omitting some elements, adding others, or both, and eleven were selected on the basis of results from legibility studies for use as stimuli. Subjects saw lists of $4,6,8$, or 11 complete letters after the degraded letter had been presented (for 4 seconds), and half the subjects saw the same list both before and after the stimulus presentation. No distractors in the list were used as stimuli. Accuracy of report (identification of the single stimulus) showed a clear effect of number of alternatives: with less alternatives accuracy was greater, even when the data had been corrected for chance: corrected score $=$ right $-(\mathrm{wrong} / \mathrm{n}-1)$. The authors' three levels of ambiguity (amount of degrading) affected accuracy, but being prepared for the stimulus by seeing the list of choices both before and after the stimulus did not given any greater accuracy than seeing the list after the stimulus presentation alone. The authors performed a second experiment with only one level of ambiguity and where 
sometimes distractors in the list came from the stimulus population ( = restricted vs. unrestricted alternatives). Numbers of alternatives in the lists were 2,4 , or 8 , and choices from these again differed significantly. Choices from restricted lists were better than from unrestricted lists, and subjects, not told of the restriction, appeared to learn this themselves. Once more the relation between accuracy and number of alternatives is an increase in accuracy with decrease in alternatives.

Garner (1962) cites Pollack's and many others' experiments which might allow one to conclude that it is response uncertainty and not stimulus uncertainty which is related to accuracy of report. Garner points out, however, that when information measures are used rather than accuracy measures, the "problem looks quite different" (p.50). Results reported in accuracy scores are, of course, meaningful, but much more meaningful when expressed relative to probability of accuracy. Psychologists usually take note of shifting response probabilities when comparing different response measures. Quastler pointed out to Pollack than most of the data in his experiment reviewed here showed constant information transmission; Pollack quoted Quastler, but remained unabashed. Finding variable accuracy in experiments using measures of variable probability is potentially a substantive result, especially where fixed practical problems need answers, but testing response uncertainty obligates an adjustment at least in a priori probabilities. In Long, Henneman \& Reid's study on degraded letters, absolute accuracy increases with decrease in number of alternatives, and this effect is significant (their Experiment I) after chance correction. If the same correction formula is used to distribute errors, uncertainty reduction decreases with decrease in number of alternatives, as do binomial test probabilities. The 
correct responses in excess of chance ( $p n$ ) actually remain fairly constant regardless of the number of alternatives.

It would seem possible to conclude that where subjects are required to identify a stimulus with the knowledge that this stimulus will be a letter, word or shape, etc., the effect of response uncertainty will be negligible. There are perhaps many situations where response accuracy is not a simple function of response probability, and even in the experiment of Long, Henneman \& Reid, comments on discriminability are well worth testing further. It is likely that is some situations there is an "optimal" amount of information "preferred" by subjects. However, if set is to be explained by either stimulus or response uncertainty, where these are distinguished, it would appear that a response explanation is not to be favoured.

\section{STIMULUS IDENTIFICATION EXPERIMENTS}

If subjects are presented with one stimulus and asked to identify it in a group of a fixed number of alternatives, set can be tested by manipulating the amount of preparation the subject receives before the stimulus presentation. Using a fixed number of alternatives keeps literal response uncertainty constant, and presenting only one unitary stimulus and requiring only one response prevents the forgetting that appears in reporting several aspects or dimensions of a complex array of stimuli.

Lawrence \& Coles (1954) conducted a stimulus identification experiment of this nature (although they called the process recognition). Subjects saw black and white photographs of common objects for brief exposures and identified them from a list of four verbal descriptions. There were three groups of subjects: one group saw the 
list of alternatives after the stimulus exposure, another saw the list before the stimulus exposure, and the third group wrote their "identifications" without seeing any list. Descriptions of the stimulus were either similar to one another, or discrete. It was hypothesised that set, or seeing alternatives before the stimulus, should improve identification from similar alternatives rather than from discrete alternatives, since subjects seeing alternatives after the stimulus use memory traces for identification and decay of these traces will be more detrimental to identification from similar than from discrete alternatives.

Results suggested alternatives aided identification, although we are again dealing with unequal response probabilities. The effect of similarity of alternatives was significant, but whether alternatives were shown before or after the stimulus was immaterial and there was no interaction. The authors argue that since alternatives in themselves facilitate identification (the first two groups of subjects scored better than the third group not seeing any alternatives), it may be concluded that alternatives work on the memory trace or else influence response variables "in the sense of making available or facilitating the occurrence of responses that otherwise would not be made" $(p .213)$. Stimuli were seen at different exposures, and accuracy of identification increased as exposure increased.

Lawrence \& Coles" experiment is a "cognitive" one since subjects seeing alternatives before the stimulus had to construct visual stimulus possibilities from verbal descriptions. A liberal interpretation of the experiment would suggest alternatives did affect identification, and if we allow this, the experiment appears to show that being prepared for the stimulus by seeing the alternatives before the exposure yields as great an accuracy of identification 
as seeing alternatives after the stimulus exposure. Preparation for perceptual processes does not appear to be relevant. Lawrence \& Coles' explanation that alternatives facilitate responses has been very popular, as may be judged from the previous section in this introduction.

Long, Henneman \& Reid (1960), like Lawrence \& Coles, used stimulus identification to test set. For Long, Henneman \& Reid's Experiment I, subjects seeing alternatives both before and after the degraded letter stimulus was presented obtained no higher accuracy than subjects seeing alternatives only after the stimulus. In their second experiment, this "temporal order" of alternatives was a significant effect, although it occurred mainly when the stimulus was identified from two alternatives (not used in their first experiment). In a third experiment, temporal order of alternatives again affected accuracy, whether subjects identified the stimulus as in the other experiments or chose between two degraded letters while monitoring a given complete letter. When choosing between two alternatives, set in the traditional sense of preparation is shown in two of these experiments. In their third experiment, the effect of preparation was greater when stimuli were less degraded, and the authors conclude that prior alternatives can augment the discriminability of important (relevant) elements in the stimulus. They also repeat Lawrence \& Coles' response explanation, although Long, Henneman \& Reid's data might be insufficient for such a conclusion.

Long, Henneman \& Garvey (1960) presented taperecorded spondees under speeded playback and found accuracy reduced when alternatives were given auditorily after the stimulus (not before, nor with visually presented alternatives), but any modality combination of before and after alternatives yielded greater accuracy than alternatives given only after 
the stimulus, which the authors found surprising. When spondees were visually presented misfocussed, there was no difference between before and after and only after alternatives. Identifying the misfocussed word from alternatives was easier than identifying it without alternatives at all: "alternatives increased the probability of each response alternative simply by reducing the number of alternatives", and the "interpretability" of the word was increased. In these two studies, it appears that set facilitated identification only with auditory stimulus presentation. An interaction in one of Long, Henneman \& Reid's experiments on degraded letters (alternatives before the stimulus facilitate identification more when degrading is less pronounced) leads to a suggestion that some types of stimulus distortion may prevent improvement by preparation when choice is made from undistorted alternatives. The subject's task in reconstructing a degraded letter or misfocussed word (both photographed and projected misfocussed) may be the more important factor in identification. Speeded auditory presentation, like tachistoscopic presentation and auditory or visual complete stimulus presentation in noise, does not physically remove stimulus characteristics, but places demands on psychological and reception processes. However, the presence of alternatives in Long, Henneman \& Garvey's visual experiment did assist identification, and if we regard this as a real assistance, the above speculation needs at least some qualification.

The experiments of Postman \& Bruner (1949) and Freeman \& Engler (1955) were stimulus identification experiments. Single set yielded better perception than multiple set in Postman \& Bruner's study (two words intersecting in a presentation), but not in Freeman \& Engler's study (one word in presentations), although set as 
general restriction was evident. Pollack's experiments (1959) also presented subjects one word for identification. For each size of response class, reduction in number of expected stimuli (message class) was a very minor effect. When message and response classes varied together (Pollack's fourth experiment), accuracy of identification fell off with increase in number of alternatives in the classes (constant information transmission). When subjects after their first response were given a second choice from their first response and the correct response (or another if their first responses were correct), accuracy was "relatively independent" of original message size. Slight reversals in Pollack's curve, however, suggest learning of the message source, in spite of Pollack's tests, and there is some decrement with increased original message size. Stimulus uncertainty is not completely eliminated.

During 1953-1955, Long and co-workers wrote many technical reports on set, and apart from the experiments repeated in 1960 and earlier reviewed, there is evidence on set in these reports only for location cuing when specific undistorted figures are monitored in a display (Long \& Lee, 1953). The conclusion one gets is that if set does facilitate perception, it is very difficult to show it.

SUMMARY REMARKS

When subjects are shown a complex stimulus with instructions to observe a particular dimension, accuracy of report on that dimension appears to be facilitated (Chapman, Lawrence \& LaBerge, Brown, Harris \& Haber, Haber). With the exception of Chapman, experimenters prefer to associate this effect with memory and response processes, 
learning or coding. Chapman would not exclude memory processes from his explanation of the effect, but did favour a selective attention or sensitised perception hypothesis: accepting a task, he said, provides a "preliminary matrix" for succeeding experience. Coding might be implicated in such a hypothesis, but distinctions will be left for later discussion. It should be pointed out here that beginning with Lawrence \& LaBerge, results that Chapman would describe as showing positive abstraction are later described as showing other processes (without any change in the results). This ambiuity about explanation is really the only source of argument!

It would seem that asking for only one report greatly diminishes the relevance of non-perceptual explanations. One report on a stimulus array, however, is perception among distractors (Archer, 1962; Hodge,1959), but theorising on this in relation to preparation has not been prevalent. A single, unitary stimulus is not forgotten in the way values of several dimensions of a stimulus array might be, and with the number of reponse alternatives constant, transmitted information can only increase or decrease with alteration of expectancies. Presenting only one stimulus, Lawrence \& Coles found no evidence for set. Long and co-workers found erratic evidence, and the irregularity may or may not have satisfactory explanations, but where their experiments did suggest preparation affected accuracy, they were surprised, and most of their results showed no effect of preparation. That set in the sense of preparation for a stimulus event should have any effect on perceptual report is a notion against the Zeitgeist. The present study intends to examine the effect of set on the identification of a single stimulus presented under tachistoscopic conditions. In this context the forgetting of one or more dimensions of a stimulus array is not relevant, and response interpretations are also not applicable. 
The present experiments examine the influence of set with a stimulus identification technique. A stimulus is briefly exposed and subjects required to identify it in a group of four alternatives. S checks one of four spaces on an answer sheet, matching the position on the sheet with the alternative he has identified as the stimulus. The position of his response is the only measure of perception used in these experiments, and is presumed to measure perception of different types of stimuli with equal accuracy. In different but not independent contexts, the measure describes identification, response selection, discrimination or recogniton, and at times these terms are interchangeable.

Set is manipulated by showing the alternatives either before or after the stimulus exposure. When alternatives are shown before, the subject is defined as being more prepared for the stimulus than when alternatives are shown after. The stimulus and the response choice are the same for both conditions, but for the subject, prior alternatives narrow the range of possible stimuli to be exposed. A difference between sequences of stimulus and alternatives is a difference of set-specificity of preparation.

Kulpe, Wilcocks, Chapman and others asked Ss for numbers, types or locations of stimulus elements in a complex display, and so made perceptual and memory spans important determinants of accuracy. Lawrence \& Coles asked Ss to select the stimulus from four alternative verbal descriptions and check off a number on answer sheets, a task in which span is not necessarily important. The present method shows $\mathrm{S}$ the stimulus among the alternatives, 
a task which does not involve span, and which, E supposes, involves less cognition or categorising than does selection from verbal descriptions.

\section{APPARATUS}

The stimulus and alternatives were shown in a threefield tachistoscope: two white fields and a temporally intervening blank field of low blue illumination. Both stimulus and alternatives are seen at a distance of $4 \mathrm{ft}$, the smallest stimuli (letters) subtending an angle of $30^{\prime}$, though internal stimulus differentiations were smaller than this. Peripheral vision is not involved. The stimulus magazine holds over 90 cards, the magazine for alternatives 30. When dispensed, the cards fall consecutively into trays. Exposed cards can be rapidly and silently dispensed though nylon flanges at the side of magazines by brass plates attached to rods within easy reach of $E$ (see Fig. 1).

Stimuli are seen through two partially silvered mirrors with reflecting illumination off; alternatives are seen reflected by the top surfaces of two mirrors with refracting illumination off. The tachistoscope cabinet was not originally designed for double lateral reversal of the second white field, and alternatives are seen at an angle from the frontal plane of $7^{\circ}$ (see Fig. 2). E did not choose any alternatives which might be confused by this slant.

Different sequences of stimulus and alternatives are selected by positioning a switch. Sequences of alternativesblank-stimulus, etc. are begun by one contact under S's or $E^{\prime}$ 's control, and complete themselves automatically.

See Appendix 1 for details. 


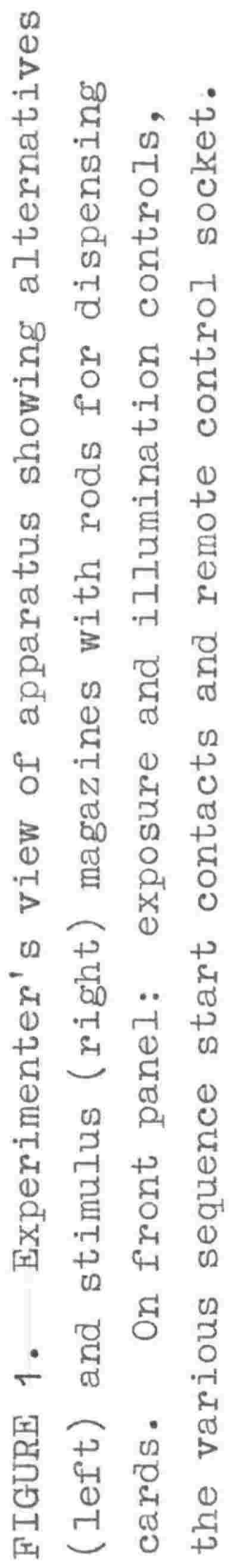




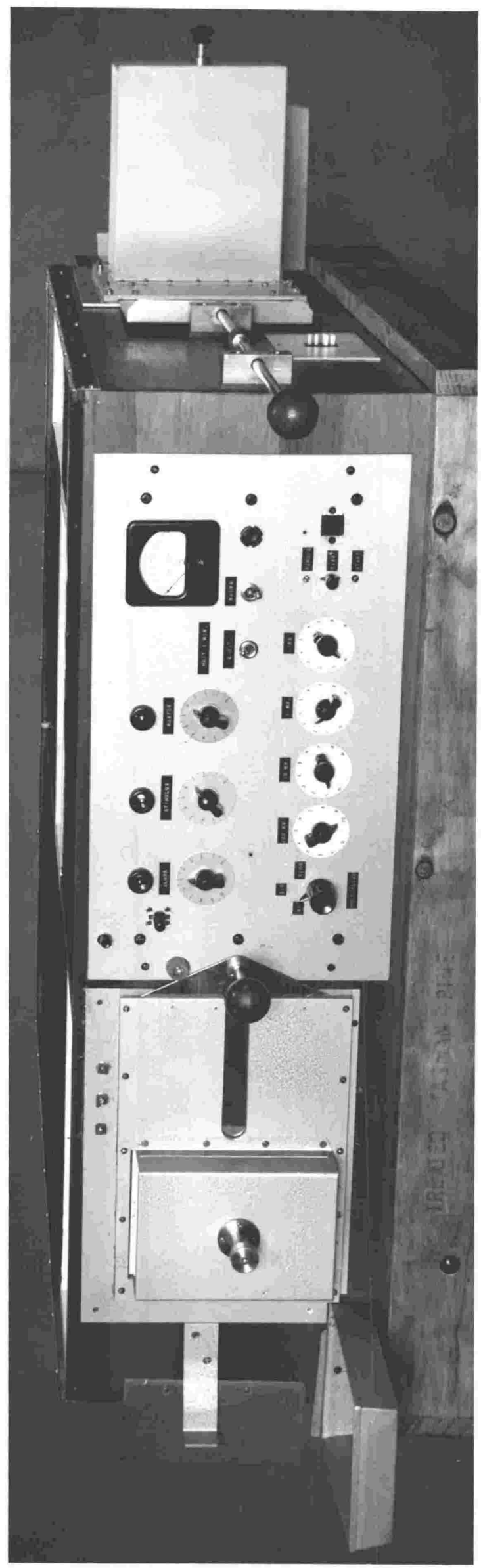




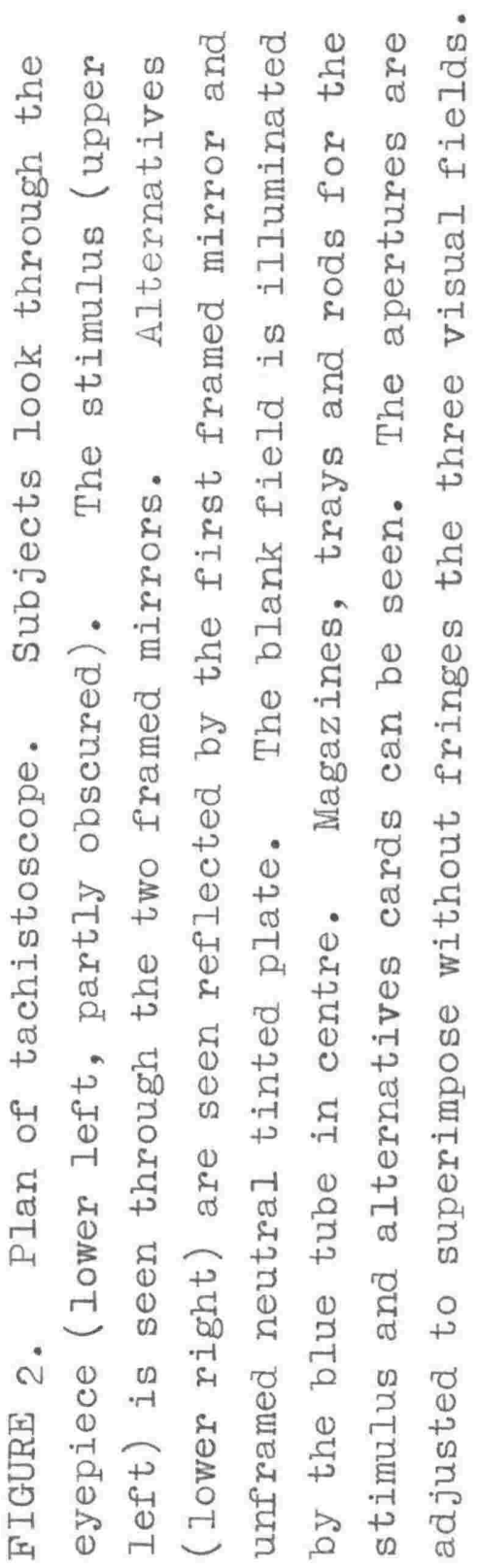




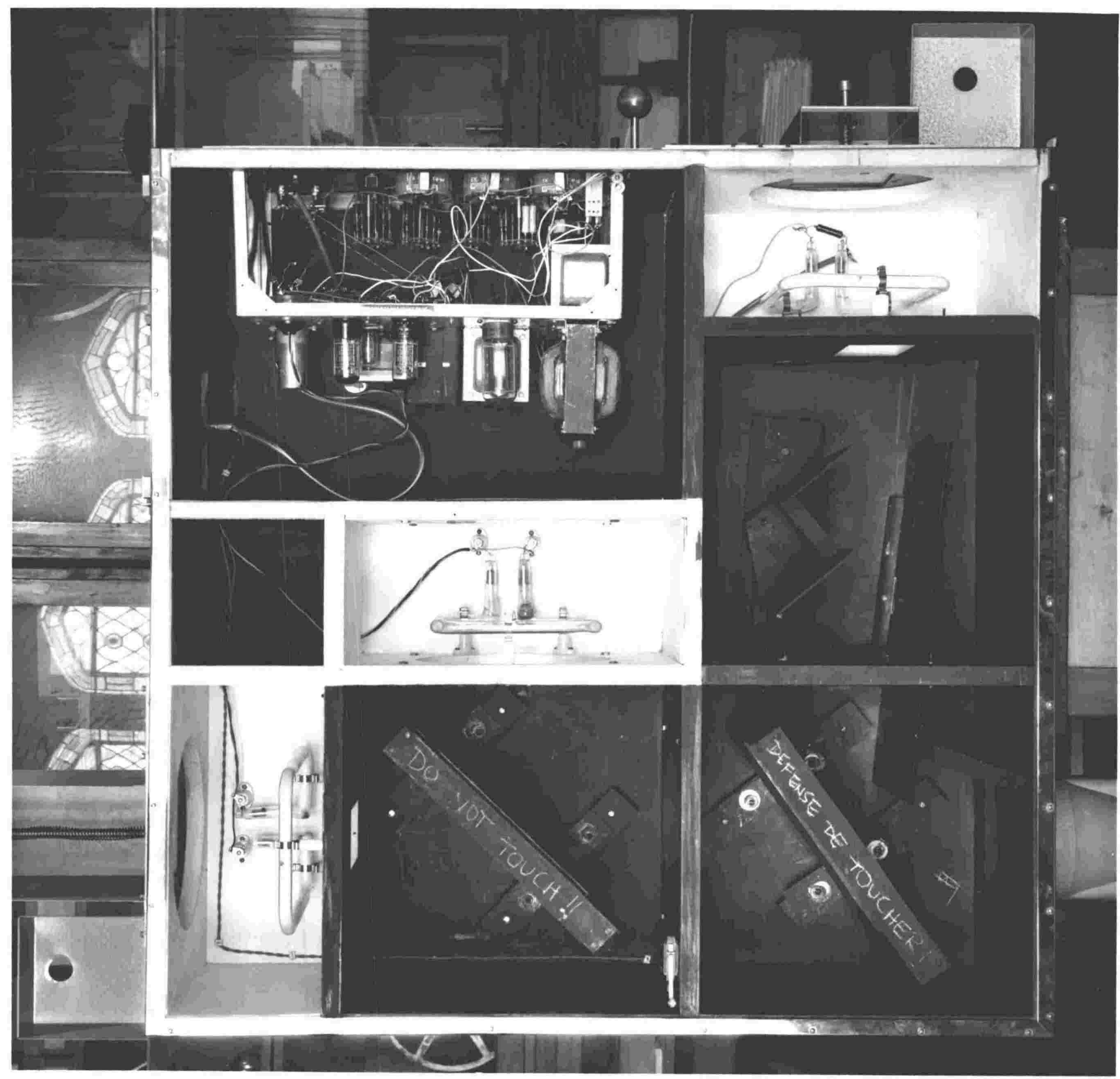


STIMULI

Six types of stimuli were shown. Shapes, pictures of common objects and two types of figures were taken from ability tests, and letters and nonsense syllables were stencilled in black ink (Feder 56 pen, Standardgraph stencil 202/6). The stimuli from test material were cut from Cattell's Culture-Free test (1944), DAT Abstract Reasoning Form A (1947), Pattern Perception Test (Penrose, 1947), Otis Primary Examination A and B (1937), and occasionally from others. The stimuli were cut without line borders, and the resulting squares of paper, measuring between 19/32" and $25 / 32^{\prime \prime}$, were pasted in the centre of $5^{\prime \prime} \times 7^{\prime \prime}$ stimulus cards of off-white Whakatane board. E lightly pencilled exact diagonals on the cards to position the stimulus in the centre, and later erased the diagonals. Sometimes E created the required stimulus by filling in an open pattern with black ink (the additions were indistinguishable). Letters were .25" high. For nonsense syllables, the middle vowel was positioned in the centre of the cards and end consonants placed naturally either side. Descriptions of the stimulus types are listed below and examples given in Fig. 3.

A. Shapes.

B. Letters.

C. Figures 1.

D. CVCs,

E. Figures 2 .

F. Pictures.
Solid forms, more regular and familiar than otherwise, and usually with more than one axis of symmetry.

All letters of the alphabet. "Open" shapes, partially filled in, which were described to $\mathrm{S}$ as "single complex figures".

Consonant-vowel-consonant trigrams with Archer (1960) values from 20 to $30 \%$.

Thin line "multiple simple figures", e.g. 3 small circles, 2 squares and an ellipse. Thin line drawings of common objects. 
A.
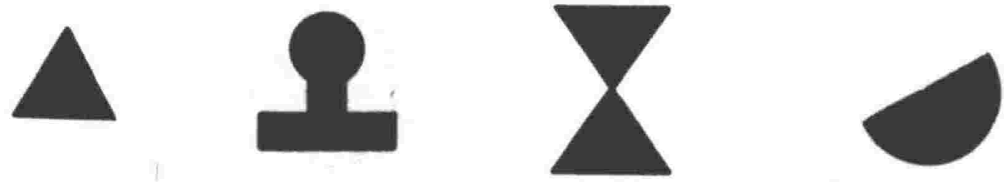

B.

G

B

$\mathrm{S}$

R

c. $\Delta$
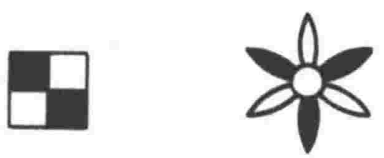

()

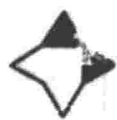

D. ZAW

QEM

XIT

YOV

WUH

E. $\begin{array}{ccc}0 & +1 \\ + & 0 & +1\end{array}$

$0^{0}$

$\overline{-1}$

$1 \mid$

F. $\rightleftharpoons$
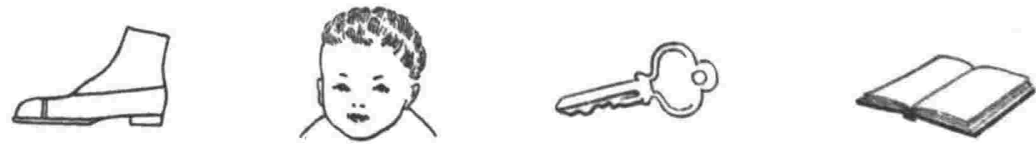

FIGURE 3. Examples of the six stimulus types. 


\section{ALTERNATIVES}

Each alternatives card showed four stimuli equidistant from its centre (Figs. 4 and 5). S's selection of the target stimulus, by checking the matching position on the answer sheet, gave him the correct response. The centre of each of the four stimuli was 1 inch above or below the card's horizontal centre line, and 1 inch to the right or left of the card's vertical centre line. An alternative occupied the approximate centre of each of four equal quadrants of the rectangular visual field, substantially separated from the card centre. Alternatives were placed by pin marks and skewed pencilled diagonals, the diagonal later removed. The target occurred in each location an equal number of times for any series of stimuli.

The three incorrect alternatives were chosen to form, with the correct alternative, a group of four equally different stimuli in order to give $S$ a real four-way response selection. The attempt to make differences between all possible pairs of stimuli equal would tend to distribute errors evenly, and keep the null probability of a partial guess being correct in the vicinity of .25 .

Half of these cards were similar alternatives, half were dissimilar. The inter-stimulus differences were smaller for similar alternatives than for dissimilar alternatives (see Fig. 6), and the comparison is taken to reflect a discrimination difficulty variable. If perception of the target at short exposures is imperfect, it is reasoned that choice from dissimilar alternatives will be easier than choice from similar alternatives. To ensure this difficulty is one of discrimination, both types of alternatives should have equal inter-stimulus differences, the differences between similar alternatives being evenly smaller than those between dissimilar alternatives. These arguments will be examined in detail later on. 

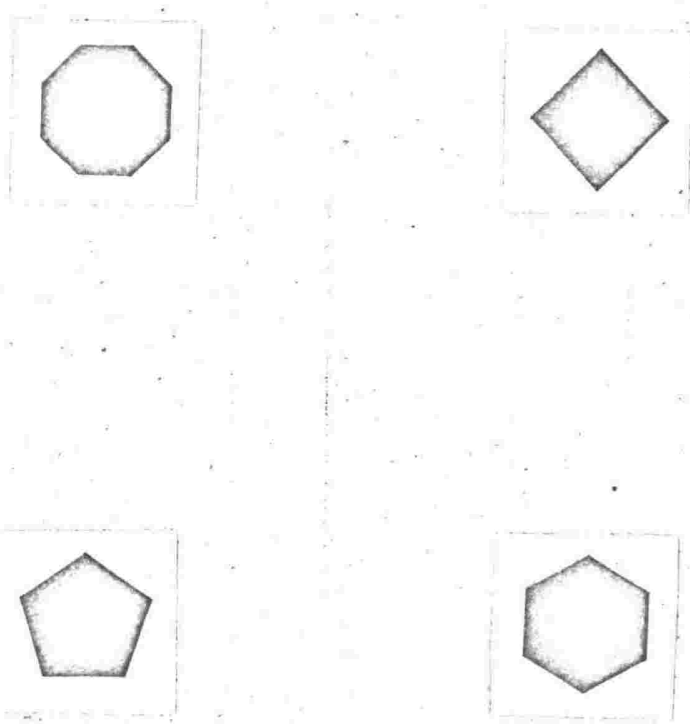

FIGURE 4. Similar alternatives for shapes, actual size. 


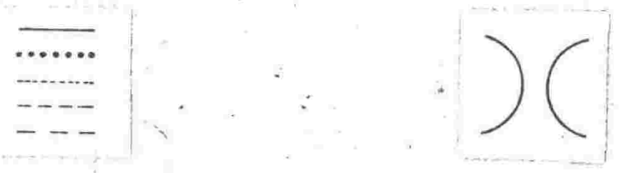

${ }_{\triangle}^{O} \underset{\Delta}{O}$

71

FIGURE 5. Dissimilar alternatives for multiple figures. 
早早

吕

$\xi$

甲

吕

呂
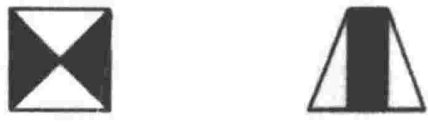

ZIF ZIK

WEQ

ZIK

ZIK

ZIX VIF

QOP

YOV
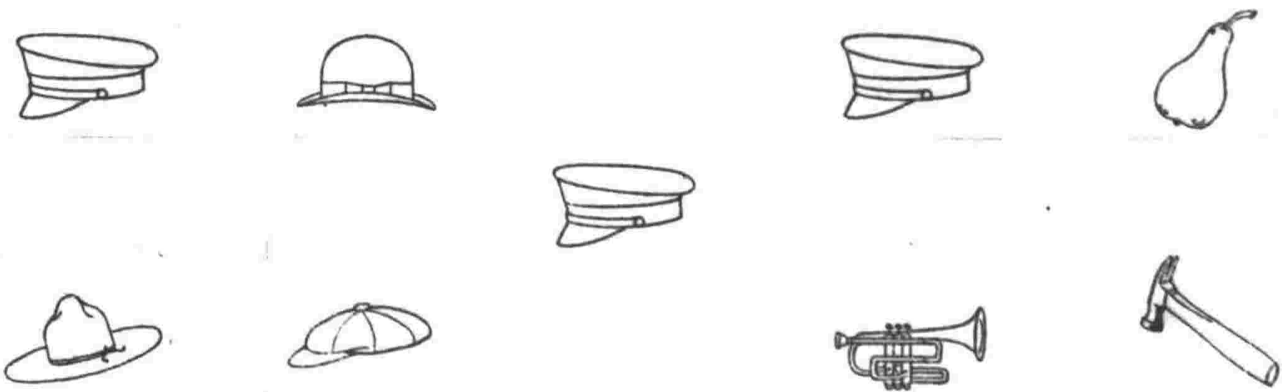

FIGURE 6. Three examples of similar (on left) and dissimilar (on right) alternatives for the same stimulus (in centre). 
RESPONSE RECORDING

Four open rectangles, 23/32" long and 26/32" high were cyclostyled on half foolscap sheets in precisely the same positions as alternatives were placed on cards. The same stencil was used for all cyclostyling. Rectangles rather than squares were chosen to given a facilitative simulation of the rectangular visual field. Booklets of the required number of pages plus one were stapled splayed for easy turning, with a folded sheet of foolscap as a cover.

SUBJECTS

Ss were male students and staff with normal vision, corrected or uncorrected. They were unpaid. Some Ss had answered a notice on the Psychology Department noticeboard; others had been approached in the Psychology laboratories, vivarium or library. After initial tests, all Ss who were asked if they would like to return to do "a second experiment" did so. Four ss were dropped because of high thresholds.

\section{GENERAL PROCEDURE}

In all experiments, subjects' own thresholds were used as exposure parameters. This procedure reduces subject differences and prevents ceiling effects.

Threshold measurement. Ss' thresholds for the types of stimulus to be seen were measured by the ascending method of limits with steps arranged for recognition at about the twelfth exposure. The stimulus was shown twice at each exposure, and steps were either .2 or .3 milliseconds (for shapes and complex figures) or $.5 \mathrm{~ms}$ (for letters, CVCs, multiple figures and pictures). E increased the step 
intervals if recognition was unusually delayed on any one trial, and if the $S^{\prime}$ s thresholds were still acceptable but irregular, extra measurements were taken.

Ss were shown a fixation spot $\left(1 / 16^{\prime \prime}\right.$ diameter $)$ and a test stimulus. Before each stimulus was presented, an alternatives card was shown on a stand beside $\mathrm{S}$, and he was told the stimulus would be one of the four alternatives. On recognition, $\mathrm{S}$ was given verbal encouragement.

Four stimuli of each type were shown. The lowest of S's four thresholds was taken as the parameter. This "threshold" is largely a function of the easiest stimulus, the other stimuli merely giving $S$ acquaintance with short exposures. The threshold is one for a somewhat masked. stimulus, emphasising outer contour perception even more than may occur without a central fixation spot. E assumed the threshold would be a lower estimate, so that fluctuation, if any, would be upward. Experiments were run at least several days after thresholds were taken.

Experimental procedure. S was first given practice with 12 stimuli he had seen on threshold trials, with exposures above and at his lowest threshold, and with the assigned sequence condition. If he saw alternatives before the stimulus, the sequence was started by $\mathrm{E}$; if he saw alternatives after the stimulus, the sequence was under his own control, the stimulus flashing as a button on a hand plunger was gently depressed. For this "After" sequence, S could thus be fully attending to stimuli. For the "Before" sequence, it is supposed that $\mathrm{S}$ can expect the stimulus with some accuracy after 12 practice trials and give the stimulus full attention. E does not suppose any differential muscular facilitation or inhibition to be important; $s$ does, however, start the After sequence himself. 
In experiments, $\mathrm{S}$ selects his repsonse from similar and dissimilar alternatives alternately. To ensure a similarity effect would not be specific to the alternatives, $\mathrm{E}$ chose for each stimulus two alternatives cards, one of each type, assigned equally to the experimental conditions.

EXPERIMENTAL DESIGN

Fixed model factorial experiments were designed with Ss to receive all stimulus and similarity conditions. Subject variances were used as error terms, and were not pooled unless increased df's were desired. Transformations were not necessary (but see Experiment I): checks on variance-covariance matrices, made only by Hartley's test, allowed nonhomogeneity hypotheses to be rejected at the .05 level with very much higher af than those indicated (Pearson \& Hartley, 1953), and Box's test, where used, gave satisfactory results.

Factorial designs were used with specific interest in sequence interactions. E has reasoned that perhaps not all perceptual conditions are facilitated by set when set might operate at all, and simple effect tests for significant two-factor sequence interactions are regarded as being in the a priori category.

In Table 1 , each $\mathrm{S}$ (rows) sees all stimulus types shown in any experiment, and selects responses from both similar (sim.) and dissimilar (diss.) alternatives for each stimulus. Four groups of $\mathrm{n}$ Ss see alternatives either Before or After the stimulus (Sequence), which is shown at either short or Iong exposures. Sequence and Exposure are between $\mathrm{S}$ effects; Stimulus and Similarity are within $\mathrm{S}$ effects. 
TABLE 1. Basic Plan of Experiments

\begin{tabular}{|c|c|c|c|c|c|c|c|}
\hline \multirow[b]{2}{*}{ Sequence } & \multirow[b]{2}{*}{ Exposure } & \multirow[b]{2}{*}{$\mathrm{S}$} & \multicolumn{2}{|c|}{ Stimulus $_{1}$} & \multicolumn{3}{|c|}{$\ldots$ stimulus $_{k}$} \\
\hline & & & sim. & diss & $\cdots$ & sim. & diss. \\
\hline \multirow{6}{*}{ Before } & short & 1 & - & - & $\ldots$ & - & - \\
\hline & & - & - & - & $\cdots$ & - & - \\
\hline & & $n$ & - & - & $\cdots$ & - & - \\
\hline & long & 1 & - & - & $\ldots$ & - & - \\
\hline & & - & - & - & $\cdots$ & - & - \\
\hline & & $n$ & - & - & $\cdots$ & $\bullet$ & $\bullet$ \\
\hline \multirow{6}{*}{ After } & short & 1 & - & - & $\cdots$ & - & - \\
\hline & & - & - & - & $\cdots$ & - & - \\
\hline & & $n$ & - & - & $\cdots$ & - & • \\
\hline & Iong & 1 & - & - & $\cdots$ & - & - \\
\hline & & - & - & - & $\cdots$ & - & - \\
\hline & & $n$ & - & - & $\cdots$ & - & - \\
\hline
\end{tabular}

The variable measured is response accuracy. For most experiments, $\mathrm{S}$ makes 12 responses in each stimulus series; the first two are practice trials (always unknown to $\mathrm{S})$, the remaining 10 are responses selected from similar and dissimilar alternatives ( $n$ (number of responses) $=5 ; n p=1.25 ; \quad x=0-5)$.

S selects his response from either similar or dissimilar alternatives for any one trial. There is usually within condition specific stimulus-alternatives balance, half the Ss seeing a particular stimulus and selecting from similar alternatives, the other half seeing the same stimulus and selecting from dissimilar alternatives. Comparison of the two halves of $\mathrm{Ss}$ in each group will test whether similar and dissimilar alternatives have the same character for both halves of Ss when seeing the same stimuli. For design purposes, the halves of similar and halves of dissimilar alternatives are regarded as equivalent. In 
most experiments, any difference between these halves will contribute to within group error terms, a between $\mathrm{S}$ effect, and consistency of stimulus and similarity variables is tested against such within group variance (the major contributor to this being individual differences).

Practice effects are assumed to be consistent or negligible. Thresholds for the different stimulus types were measured in the order stimuli are listed above, A to F; practice trials are run in this order; and stimuli are administered in the same order. All Ss make responses to the same stimuli at equal levels of practice. E suspects practice to be of little importance, though where the same $\mathrm{Ss}$ are used in later experiments, comments on practice effects will be made.

\section{DISCUSSION OF VARIABLES USED IN THE PRESENT STUDY}

Sequence. Set or preparation is defined by the sequence factor. If Ss seeing alternatives before the stimulus are able to identify it better than ss not seeing alternatives until after the stimulus, this sequence effect is taken to demonstrate that set facilitates identification. Set cannot be said to facilitate identification should there be no difference between the two sequence conditions. The preparation that Before sequences allow is highly specific. All Ss are familiar with the stimulus types and on any one trial know what sort of stimulus will be presented. Ss seeing alternatives before the stimulus, however, have more specific expectations: instead of being prepared merely for a stimulus category, they can expect one of four alternative stimuli. This increased specificity of expectation allows $\mathrm{S}$, in the traditional exposition, to selectively attend to those aspects of the stimulus which may facilitate its identification. 
The other variables in this study were chosen to create differing perceptual conditions that may clarify the nature of set. $E$ has initially assumed that set may benefit identification only under certain conditions, and variation in these conditions is made to allow sequence interactions.

Exposure. Stimuli are shown at short and long exposures determined by S's threshold for the stimulus presented. A short stimulus exposure should reduce the amount of stimulus information and hence accuracy of identification from alternatives.

Similarity. The two types of alternatives possibly create a discrimination difficulty variable, selection from similar alternatives being less accurate than from dissimilar alternatives.

Stimulus. The six stimulus types emphasise, superficially, different perceptual factors: from size and contour of areal mass in shapes to numerosity and pattern in Multiple Figures. In CVCs there is a less unified type of stimulus, where separate elements (letters) may be independently perceived.

Interval. In some experiments $\mathrm{E}$ attempted to create forgetting of the stimulus by increasing the interval between the stimulus exposure and the presentation of alternatives. This enforced delay of response may show reduced accuracy of identification. Interval will be discussed in later experiments.

Threshold, a subject factor, groups high and low threshold Ss separately in some experiments to avoid obscuring the possibility of high threshold ss obtaining high identification scores because of the relation between 
absolute exposures and identification accuracy. In most experiments, however, care is taken to form experimental groups of Ss so that group threshold totals are equal, preventing between group variation in accuracy because of threshold differences. Employing Ss' thresholds is desirable since subject differences are thereby diminished, and with the increased homogeneity of experimental units, less subjects need be run without jeopardising measurement precision. E does not consider this idiographic equation eliminates the relationship between exposure in milliseconds and accuracy, and regards as still necessary a distinction between high and low thresholds.

Summary of discussion. Exposure, similarity and interval are easily manipulable variables which allow a variety of perceptual conditions with known or suggested effects. The stimulus types offer a range of material emphasising different perceptual skills, although overall difficulty variations are excluded by exposing stimuli at values related to specific stimulus thresholds. Experiments should allow sequence to interact with the above variables, demarcating the influence of set if set at all influences perception.

Note on motivation. S's major task is to perceive a briefly exposed stimulus every 20 seconds or so. This task is apparent to him ( $h$ is presence as a subject is pointless otherwise), and we might expect optimal motivation for it. Exercising acute discriminative skill in choosing the stimulus from alternatives is to some extent satisfying, and the difficulty of the task ensures close attention. Confidence in having made a correct selection is likely to be strong reinforcement, especially when task difficulty is realised. Many subjects appear to have a fasciniation for tachistoscopic 
exposures, spontaneously expressing enjoyment and interest after completing threshold trials (although verbal reinforcement is given in these trials). The perceptual material itself is not uninteresting, postulates of curiosity theorists being apposite. However, Ss are not paid for their participation. Some ss were obtained by approaching students in the Psychology Department, and obliging $\mathrm{E}$ was probably the major reason for the willingness of several of these students to be Ss. Although the motivational factors above might apply to all Ss, those assigned to difficult perceptual conditions may have experienced frustration. E warned all Ss that experimental trials would be "more difficult" than threshold or practice trials, and S's comments between stimulus series were heard solicitously. E supposes this warning and implicit morale-boosting prevents any frustration depressing accuracy scores.

We may assume the structure of the situation creates a focussing of effort onto the central task, and this can be considered adequately if not optimally motivating. 
The design is for a $2 \times 2 \times 6 \times 2$ factorial experiment; $p$ (sequence) and $q($ exposure) are between $\mathrm{S}$ effects, $r$ (stimulus) and s (similarity) are within $\mathrm{s}$ effects. The basic plan given in Table 1 fits Experiment I. All 6 types of stimulus are used: shapes, letters, complex figures, CVCs, multiple figures and pictures, and $\mathrm{S}$ selects responses from similar and dissimilar alternatives. One sequence and one exposure condition is administered to each $\mathrm{S}$. For a long exposure condition, stimuli are seen at S's threshold value for the particular stimulus; for a short exposure condition, 8 of this threshold value is used. For example, if S's threshold for shapes is $1.6 \mathrm{~ms}$ and he is assigned to a short exposure condition, shapes will be exposed at $1.3 \mathrm{~ms}$ ( $0.1 \mathrm{~ms}$ is the minimum calibration).

Subjects see alternatives for 10 seconds either before or after the stimulus exposure, the interval between the two presentations being 1.8 seconds (stop watch timing average, \pm 0.1 sec.). The interval is long enough for afterimages to decay (for illumination used and image articulation required for correct identification), an interval longer than Sperling's estimate of short term memory measured by a partial span reduction method, and much longer than Averbach \& Coriell's (1959) estimate of "visual storage time" derived from selected stimulus masking (both these estimates using letters as stimuli). Decay of stimulus information is presumed to affect After sequences only. With Before sequences, having seen the alternatives for 10 seconds, $S$ is presumed able to make a choice very soon after the stimulus exposure. Any information decrement, it is assumed, will refer to alternative location for a Before sequence.

The 1.8 second interval, it may be taken, ensures that 
we are not measuring retinal phenomena or ephemeral information likely to be dependent on illumination parameters. Whether or not immediate storage of information for simple, one-response tasks like the present one requires a more subtle analysis, will be left to later experiments.

SUBJECTS

Sixteen Ss were first split into two groups, I and II, 2 Ss of each of these groups to receive the same condition. For any particular condition, group I Ss select their response from similar alternatives for either odd or even numbered stimuli, while group II Ss select from dissimilar alternatives for stimuli with the same numbers. The I and II subgroups of $\mathrm{pq}$ groups of $\mathrm{n} \mathrm{Ss}$ were matched according to the feasible relation of thresholds and accuracy measures: the I and II groups were ranked 1 to 8 in each group from highest to lowest threshold totals, and $\mathrm{S} 1$ placed with $\mathrm{s} 8$, S2 with S7, etc., to form 4 subgroups of group I and 4 subgroups of group II. The S1 and S8 of I were placed with $\mathrm{S} 4$ and 55 of II, etc., forming the 4 (pq) experimental groups, and these groups were assigned at random to the pq experimental conditions. This assignment avoids the possibility of between group variation due to correlation between threshold and accuracy measures.

PROCEDURE

$\mathrm{S}$ is asked to sit down and note the fixation spot in the blue blank field, and given a test stimulus in the assigned sequence condition. Practice is given with 12 stimuli exposed above and at S's threshold values, and responses made in a booklet. If the initial practice stimulus is not seen (on questioning), this or the next stimulus is shown at a longer exposure to convince $\mathrm{S}$ there 
are no vexierversuche, and to offer some reinforcement. On later trials in the practice series where $\mathrm{S}$ declared his response might be a guess, he was told a forced choice would be requested. Some Ss felt more or less certain that they had selected the correct alternative for all practice stimuli, but accuracy was never $100 \%$.

$S$ was then given a new booklet and told he would see 12 shapes (etc.) "at your threshold, which will be harder to see than the stimuli in the practice trials", and that a response is required on every trial.

Sessions of practice trials and the 6 stimulus series took a little less than an hour. Between each stimulus series a short break was given, and with each new series E said: "Now I'd like to show you some nonsense syllables (etc.); these will be very much like the ones you saw before" etc.

Booklets were scored directly and locations of $\mathrm{S}^{\prime} \mathrm{s}$ responses entered into a score sheet for checking correct and incorrect choices.

All ss know what type of stimulus will be shown, and the set or preparation that Before sequences allow the $S$ is quite specific.

RESULTS

Distributions of main effects were adequately normal, as were those for interactions, these latter by inspection. Between error term components were not homogeneous by Hartley's test, and the heterogeneity appeared to be due to one $S$ in the After sequence, short exposure condition. Logarithm and reciprocal transformations did not reduce this 
heterogeneity to within reasonable limits and regretfully $\mathrm{E}$ decided to run another $\mathrm{S}$. Other error components were homogeneous with the excluded $\mathrm{S}$, and all errors were homogeneous with the new S. (See Appendix 2 for details about the excluded S.) The within error mean squares were also fairly even ( $p>50$, Bartlett's test), although Ss appear to respond to similarity a little more regularly. The analysis is presented in Table 2 with error terms arranged for convenient observation.

Main effects of exposure and similarity are significant, and the stimulus types differ significantly (multiple figures easiest, shapes hardest). There is little evidence for a sequence effect. E concludes that set has no noticeable effect on accuracy when measured with the present method.

Two interactions are significant: sequence $x$ exposure and exposure $\mathrm{x}$ similarity, and there is a tendency for a sequence $x$ similarity interaction $(F=4.68, F .05$ $(1,12)=4 \cdot 75)$.

The sequence $x$ exposure interaction (Fig. 7) shows that an exposure difference exists only for the After sequence. At short exposures the After sequence yields less accuracy than the Before sequence, the converse being the case for long exposures. It might be speculated that two factors, set and forgetting, are involved in this interaction. The sequence difference at short exposures might be attributed to set (the difference is not significant); the sequence difference at long exposures (also not significant) might be attributed to forgetting of alternative location by the Before sequence group. Forgetting cannot apply to the After sequence group, since $\mathrm{S}$ is able to ascertain his intended choice by glancing back at the alternatives before the 10 seconds exposure has 
TABLE 2. Experiment I: Analysis of Variance.

\begin{tabular}{lrrr}
\multicolumn{1}{c}{ Source } & df & MS & F \\
\hline Between Ss & 15 & & \\
A (Sequence) & 1 & .1302 & \\
B (Exposure) & 1 & 13.5469 & $5.69 *$ \\
AB & 1 & 12.5052 & $5.25 *$ \\
Ss/Groups & 12 & 2.3802 & \\
Within Ss & 176 & & \\
C (Stimulus) & 5 & 6.8802 & $5.19 * * *$ \\
AC & 5 & 2.2052 & 1.66 \\
BC & 5 & 1.6469 & 1.24 \\
ABC & 5 & .5052 & \\
C X Ss/Groups & 60 & 1.3260 & \\
D (Similarity) & 1 & 13.5469 & $27.57 * * *$ \\
AD & 1 & 2.2969 & 4.68 \\
BD & 1 & 7.1302 & $14.51 * *$ \\
ABD & 1 & 1.2552 & 2.55 \\
D X Ss/Groups & 12 & .4913 & \\
CD & 5 & .5719 & \\
ACD & 50 & 1.3228 & \\
BCD & 5 & 1.8719 & 1.41 \\
ABCD & 5 & .7302 & \\
CD x Ss/Groups & 5 & 1.3552 & 1.02 \\
TotaI & 50 & & \\
\hline \hline
\end{tabular}

Note. *: p<.05; **: p<.01; ***: $p<.001$, in this and in all other Tables.

completed. The speculation appears to imply that forgetting is more important than set for the higher levels of accuracy that are possible with long exposures. (The nonsignificant sequence $\mathrm{x}$ similarity interaction does not 


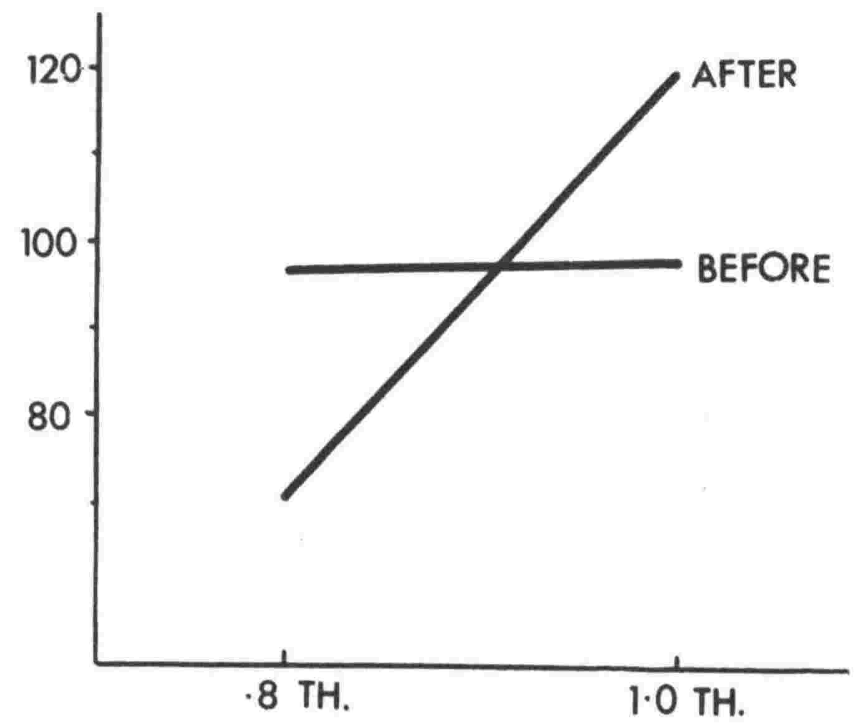

FIGURE 7. Experiment I: Sequence $x$ Exposure (.8 and 1.0 of $\mathrm{S}^{\prime} \mathrm{s}$ stimulus threshold) interaction. Accuracy totals on ordinate.

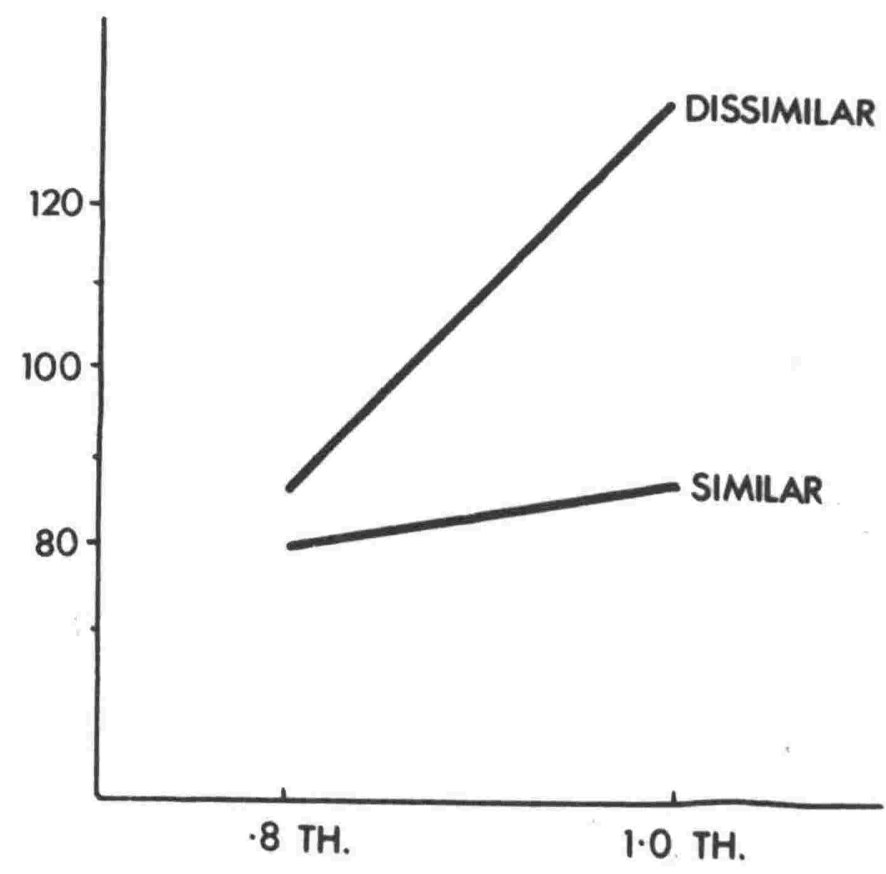

FIGURE 8. Experiment I: Exposure $x$ Similarity interaction. Accuracy totals on ordinate. 
contradict this interpretation: this interaction shows a greater similarity difference for the After sequence.)

Accuracy was very low for the group seeing alternatives after the stimulus at exposures of .8 of their thresholds. For shapes, letters and complex figures, scores were less than chance, and only for multiple figures and pictures did accuracy significantly exceed chance.

The exposure $\mathrm{x}$ similarity interaction is plotted in Fig. 8. Accepting that increasing exposure facilitates perception, this interaction might suggest that the similarity factor is indeed a discrimination difficulty factor as $\mathrm{E}$ had intended. Increasing exposure does not facilitate choice from similar alternatives as much as it facilitates choice from dissimilar alternatives. significant simple effect occurs only for dissimilar alternatives, p<.01, although a posteriori.) Discriminative choice prevent accuracy increasing at long exposures.

Thresholds and accuracy of identification. The correlation between log thresholds and accuracy for subject totals is .539 ( $N=16)$, and drops, because of the matching procedure, to .023 for subgroup totals ( $N=8$, I and II $p q$ ). Summing threshold measures and accuracy scores over Ss (i.e. obtaining stimulus totals) gives a correlation of .946 $(N=6)$; .972 for accuracy from similar alternatives, and .898 for accuracy from dissimilar alternatives. It would seem, therefore, that while between effects would not be reduced by adjustment for thresholds as covariates, the stimulus effect should be greatly reduced by such an adjustment. (The similarity effect, a "within stimulus series"measurement, is not altered by the adjustment, both levels of the factor having the same threshold covariates for each stimulus with any one S.) 
In the following summary table, the between and within errors had equal within group regressions (F 1). TABLE 2. Experiment I: Analysis of Covariance. Source (adj•) df MS F

\begin{tabular}{lrrr}
\hline A (Sequence) & 1 & .1201 & \\
$\mathrm{~B}$ (Exposure) & 1 & 10.8178 & $7.75 *$ \\
$\mathrm{AB}$ & 1 & 12.5052 & $8.97 *$ \\
$\mathrm{Ss} /$ Groups & 11 & 1.3944 & \\
$\mathrm{C}$ (Stimulus) & 5 & .7287 & \\
$\mathrm{AC}$ & 5 & 2.0320 & 1.55 \\
$\mathrm{BC}$ & 5 & 1.6207 & 1.23 \\
$\mathrm{ABC}$ & 5 & .4485 & \\
Residual & 59 & 1.3139 & \\
\hline \hline
\end{tabular}

Variance due to stimuli has largely disappeared; otherwise the only substantial change has been the improvement in the between $\mathrm{S}$ error term. The planned. simple effect tests on the $A B$ interaction are still, however, not significant.

Alternatives. Comparing similar and dissimilar alternatives for a particular stimulus is a comparison of subgroups I and II. A direct comparison is unfortunately not possible as E administered the wrong alternatives to one $S$ in the original subgroup II. Although similarity remains balanced, comparison of alternatives for individual stimuli becomes a somewhat untidy affair, and less powerful a test than was planned. It is still possible, however, to gain an idea of the comparison in changes in proportions $(x / 7$ and $x / 9)$ of correct responses chosen from either alternatives card for the same stimulus. Considering proportion changes of less 
than 100 (e.g. from $4 / 7$ to $5 / 9$, or $7 / 9$ to $5 / 7$ ): there are 19 increases in the expected direction (an increase from similar to dissimilar), and 17 in the reverse direction (a decrease from similar to dissimilar). Of the proportion changes greater than $.100,20$ are in the expected direction and 4 in the reverse direction (the latter perhaps indicating faulty construction of alternatives, although some reversals might be expected by chance).

The two administrations of alternatives can be compared by testing the equivalence of subgroup totals for either similarity condition for each stimulus series. This comparison is no longer specific to each stimulus, but applies to groups of 5 alternatives. A two-sample test on the 24 subtotals appears to be appropriate. Using expected values derived from similarity and stimulus series sidetotals, $\chi^{2}=3.179$ (af 10), p >.95, suggesting the character of the two administrations is much the same.

Similarity. Although both similar and dissimilar alternatives offer $\mathrm{S}$ a choice from four possible stimuli, and hence a superiority of choice from dissimilar alternatives cannot be a function of literal response restriction, or probability, it remains to show that an equal number of possible choices are in fact available in selection from both types of alternatives. The construction of dissimilar alternatives may be such that on many occasions at least one particular choice is immediately discounted as a possible answer, the eventual discrimination being between three or less alternatives. While it is likely that this process is the normal one of selection from several alternatives, should the same dissimilar alternative be avoided by most subjects, we would in effect be reducing the probability of error, which contingency must then be a potential cause of superior accuracy when choosing from dissimilar alternatives. As mentioned earlier, both types 
of alternatives were constructed so that differences between alternative stimuli on each card would be even. The comparison between similar and dissimilar alternatives might then be said to be one of discrimination difficulty, and the exposure $\mathrm{x}$ similarity interaction suggests this.

The problem was recognised by Lawrence \& Coles, who took the 18 photographs for which there were five or more errors for both types of alternatives and compared uncertainty measures derived from the distributions of responses among the three wrong alternatives. This "rough check" (and it is rough) showed that the three wrong choices were more evenly selected from dissimilar alternatives, although the authors doubted the importance of the difference.

A test of the imbalance must be independent of $\mathrm{N}$ since there are more errors with choice from similar alternatives. Chapanis' exact test (1962, Gridgeman,1964) appeared to most approximate the requirements. The probabilities of the distributions of responses among the three wrong alternatives for each card were summed separately for similar and dissimilar alternatives to give the minimum $\mathbb{N}$ for a $\chi^{2}$ test. One restriction on the summing criterion was observed: the distribution 3,2,1 $(p=.88)$ occurred 11 times (10 similar, 1 dissimilar), and the distribution 2,1,0 $(\mathrm{p}=.78)$ occurred 10 times ( 3 similar, 7 dissimilar). E regards these two probabilities as being equal since no values in between are possible with the low $N$, and the test should be independent of $N \cdot \chi^{2}=2.321$ (df 5), p >.80. Without the summing restriction, $\chi^{2}=11.239$ (df 6 ), $.10>p>.05$; and testing only $p^{\prime}$ s less than $.50, \chi^{2}=1.178$ $($ af 2$), p>.50$.

There are 3 alternatives for which the error distributions are significantly uneven (all 4,0,0, p =.037). 
These are for letter stimuli, and all are dissimilar alternatives (the correct letter in brackets, the incorrect letter underlined): ( $\mathrm{S}) \mathrm{T} W \underline{\mathrm{D}}, \mathrm{L} \underline{\mathrm{D}}(\mathrm{K}) \mathrm{U}$, and $\mathrm{F} \underline{\mathrm{O}}(\mathrm{W}) \mathrm{S}$. While 3 significant results occur by chance and problems of N complicate interpretation, E cannot reject the possibility that these dissimilar alternatives are actually less equally chosen than others. The reader may judge that the inter-alternative differences are not clearly equal in these alternatives, and it is possible that the fixation spot contributed to S's confusion.

The mean of wrong choices for alternative stimuli that were chosen at least once may give a suggestion of how wrong responses pile up in less than 3 alternatives. These means are: for similar 2.0191, for dissimilar 1.9111. The variances: for similar 0.8213, for dissimilar 1.0217 $(F=1.24, F .05(134,156)=1.35)$. The means may superficially indicate greater evenness of choice from dissimilar alternatives $(z=0.955)$, but with less total errors to be distributed, the picture given is not, $\mathrm{E}$ would think, as representative as that given by comparing the variances. The dissimilar variance is substantially greater, with less total errors to be distributed, and with both $\mathbb{N}^{\prime}$ s high enough to avoid marked changes in variance with changes in $\mathrm{N}$.

One might conclude from this cumbersome analysis that there is a slight suggestion of greater unevenness in choices from dissimilar alternatives. An explanation for any such suggestion might be subject consistency in rejecting one or more alternatives, and the inequality of interalternative differences might underlie this. One cannot state any conclusion unequivocally, since no null hypothesis has been rejected. A more efficient attack on this problem is possible in later experiments. 
SUMMARY

1. Whether alternative response choices in an identification task are shown before or after a brief stimulus exposure, accuracy of identification remains unaffected. There is little evidence for any benefical effect of preparation.

2. Accuracy increases with increasing exposure.

3. Accuracy is better when selection is made from dissimilar alternatives, increasing when exposure is increased; but selection from similar alternatives is not improved by increasing exposure. Similarity may reflect discrimination difficulty, although analysis of errors might suggest that discrimination from dissimilar alternatives is a somewhat different process than discrimination from similar alternatives, and/or that some dissimilar alternatives were poorly constructed.

4. The sequence $x$ exposure interaction is significant: at short exposures alternatives before the stimulus yield greater accuracy, at long exposures alternatives after the stimulus yield greater accuracy. These differences may show set and forgetting respectively.

5. Accuracy for the different stimuli is directly related to stimulus exposures. There are no stimulus interactions. 
Experiment II tests the suggestion made in Experiment I that forgetting may occur with Before sequences. In Experiment I Ss in this sequence condition first saw alternatives for 10 seconds, then the briefly exposed stimulus, and were then required to check off the alternative which most closely matched the stimulus as perceived. The task requiredperfect retention of the four alternatives in their location if the sequence comparison is to be purely one of the influence of set. An interaction in Experiment I suggests some forgetting did take place in the Before sequence, and if the suggestion has any foundation in fact, the experiment cannot be said to be an adequate test of the major hypothesis: specific preparation for a stimulus assists identification. Forgetting of the requisite information for correct responding may annul any preparation effect that Experiment I Before sequences gave.

\section{METHOD}

The Before sequence for Experiment II shows $S$ the alternatives for 5 seconds prior to the stimulus exposure and again for 5 seconds after the stimulus exposure. Any forgetting of alternative location is eliminated. A sequence difference would now be due to the extra alternatives presentation for the Before sequence. Ss in the After sequence condition see alternatives for 5 seconds after the stimulus exposure. All Ss know the type of stimulus to be shown (shapes, letters, etc.); the preparation in Before sequences is quite specific.

Exposures for alternatives were 5 seconds since $\mathrm{E}$ observed very few occasions when $S$ took longer than this time in Experiment I, and many Ss said they thought the 10 
second exposure was unnecessarily long. In Experiment II, Ss had nearly always made their response after seeing alternatives (after the stimulus) for about one second.

Stimuli. Four types of stimuli are shown: shapes, letters, complex figures and CVCs. There are 22 stimuli in each series, the first 2 practice stimuli, the other 20 selected from either similar or dissimilar alternatives (possible number correct $=10 ; n p=2.50 ; x=0-10)$. The extra stimuli and alternatives were prepared as before, the 12 stimuli of the relevant stimulus series of Experiment I forming the first part of the 22 stimuli of stimulus series for Experiment II. E ran the extra stimuli to see if reliability of measurement increased when each datum is a sum of 10, rather than 5 responses, and to make possible some estimate of practice or fatigue effects (though halves of stimulus series were not inverted for balance). The stimuli of Experiment I not used were those for which extras were not available. E had nearly exhausted the supply of these stimuli already, extra samples of requisite homogeneity (thin lines, small size, etc.) not being available. Session length is about one hour, with 2 to 3 minute breaks between stimulus series.

Subjects. 24 new male ss were run. Threshold totals were used to assign Ss to either a "high threshold" or "low threshold" condition. The summed thresholds for the "low" group ranged from $10.1 \mathrm{~ms}$ to $14.5 \mathrm{~ms}$, and for the "high" group from $16.0 \mathrm{~ms}$ to $29.4 \mathrm{~ms}$. High and low thresholds formed a full "threshold" factor, a between $\mathrm{S}$ effect.

The similarity factor is the same as for Experiment I. All Ss see all stimuli, half the Ss see one type of alternative for any stimulus, the other half of Ss see the other type of alternative. Administrations of alternatives were assigned to either high or low threshold Ss. 
The interval between stimulus and alternatives is again 1.8 seconds. Experiments are much the same except for the second presentation of alternatives for Ss assigned to the Before sequence. The levels of the exposure factor are again .8 and 1.0 of $\mathrm{Ss}^{\prime}$ stimulus thresholds. Distinguishing high and low threshold subjects eliminates the need for matching subject groups on thresholds. The booklets of 23 pages were scored as before. The design is for a $2 \times 2 \times 2 \times 4 \times 2$ factorial experiment, $p$ (sequence), $q$ (exposure), and $r$ (high or low threshold) are between $\mathrm{S}$ effects, and s (stimulus) and (similarity) within $\mathrm{s}$ effects, all ss receiving both similarity conditions of all stimulus types.

\section{RESULTS}

All errors were homogeneous by Hartley's test, critical df for observed $F_{\max } .05$ values being far greater than required. The within errors were also of the same order (Bartlett), though as in Experiment I, there is a smaller error for similarity. The stimulus variancecovariance matrices, the only ones worth testing (more than two levels), were equal and symmetrical by Box's test $\left(\boldsymbol{\chi}^{2}\right.$ for equality $=36.027$ (af 30$), \mathrm{p}>.20, \chi^{2}$ for symmetry $=$ 4.128 (af 8), p>.50).

The threshold effect and all interactions with the threshold factor are not significant, none reaching $p=.10$ except the threshold $\mathrm{x}$ stimulus $\mathrm{x}$ similarity interaction which represents conditions specific to subjects' responses to individual administrations for each stimulus series. E considers the data allow dropping the threshold factor from the model and the results are presented as a four factor experiment. The original analysis is presented in Appendix 3. 
TABLE 4. Experiment II: Analysis of Variance.

\begin{tabular}{lrrc}
\multicolumn{1}{c}{ Source } & df & \multicolumn{1}{c}{ MS } & F \\
\hline Between Ss & 23 & & \\
A (Sequence) & 1 & 121.9219 & $24.50 * * *$ \\
B (Exposure) & 1 & 13.5469 & 2.72 \\
AB & 1 & 1.8802 & \\
Ss/Groups & 20 & 4.9760 & \\
Within Ss & 168 & & \\
C (Stimulus) & 3 & 6.0330 & 1.69 \\
AC & 3 & 12.3941 & $3.48 *$ \\
BC & 3 & 1.9635 & \\
ABC & 3 & 2.7691 & \\
C X Ss/Groups & 60 & 3.5649 & \\
D (Similarity) & 1 & 68.8802 & $39.71 * * *$ \\
AD & 1 & 3.2552 & 1.88 \\
BD & 1 & .4219 & \\
ABD & 1 & 4.3802 & 2.53 \\
D X Ss/Groups & 20 & 1.7344 & \\
CD & 3 & 2.5469 & \\
ACD & 30 & 3.0608 & 1.15 \\
BCD & 3 & 1.6163 & \\
ABCD & 3 & 1.7115 & \\
CD x Ss/Groups & 3 & & \\
TotaI & 30510 & \\
\hline \hline
\end{tabular}

There is a very clear influence of set in this experiment. There is one sequence interaction, sequence $\mathrm{X}$ stimulus: all stimuli benefit from prior presentation of alternatives, but the sequence difference is greatest for CVCs $(p<.001)$ and least for letters $(p<.05)$. The similarity effect is also pronounced, but there are no interactions. 


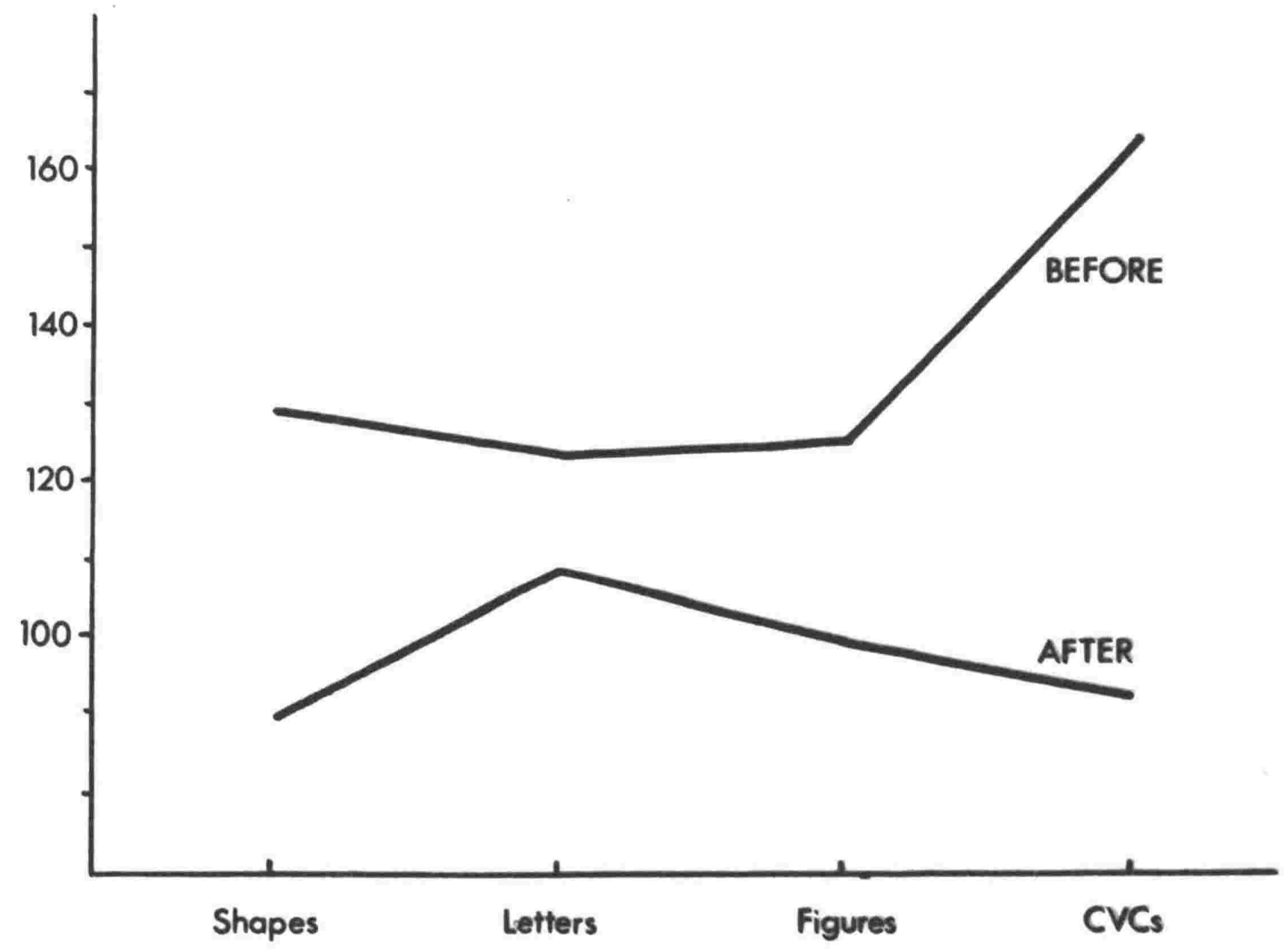

FIGURE 9. Experiment II: Sequence $\mathrm{x}$ Stimulus interaction. 
If it can be assumed that of the stimuli shown CVCs are the most complex, or carry the most information, the sequence $\mathrm{x}$ stimulus interaction is not surprising. But with analogous reasoning, there should perhaps be a sequence $\mathrm{x}$ similarity interaction-Before sequences allow $S$ to give attention to less obvious details which carry much information necessary for correct identification-and this interaction is hardly apparent $(F=1.88, F .05(1,20)$ = 4.35). The complexity of CVCs appears to be perceptually distinct from complexity (similarity, ambiguity) of the similarity factor. These points will be discussed later.

Halves. Accuracy scores for the two halves correlate .67 $(N=24)$. For individual stimuli (shapes, letters, complex figures and CVCs) the correlations are .38, .29, .47, and .62 respectively. These show less reliability than expected. Scores are a little higher for the second half, $t=0.052$, with the corrected S.E. $t=0.939(\mathrm{df} 23)$, and higher scores occur with all stimuli (3, 10, 16, and 2 more respectively, each half out of a possible 120 for each stimulus). The figures might be taken to suggest a slight practice effect, and $\mathrm{E}$ broke down the figures for Before and After sequences separately. It was the Before sequence which showed improvement in the second half $(t=1.466$ $($ df 11), .10>p>.05), the After sequence scores showed a total of 1 less correct response in the second half. Before halves correlated .72 , After halves .25 (both $\left.\mathbb{N}^{\prime} \mathbf{s}=12\right)$.

The practice effect evident for the Before sequence does not seem attributable to unequal difficulty of the halves (unless one postulates a compensating fatigue effect for the After sequence). Learning to utilise information given by prior alternatives may not be complete in practice trials, although the practice effect did not develop as the session advanced (by inspection). It seems that increasing 
use of differentiating characteristics of prior alternatives for each new stimulus series offers the most plausible explanation.

Error analysis. Testing the distribution of errors with Chapanis' test shows that of the 160 alternatives cards, errors were significantly uneven in 6 cases ( 1 at the .01 level): 2 dissimilar, 4 similar; 2 shapes, 2 letters, 1 figure and 1 CVC. Even distributions $(p=1.00)$ occurred with 15 similar and 22 dissimilar alternatives (perhaps partly a function of the number of errors to be distributed), and for probabilities between .150 and.194 there were 8 similar and 1 dissimilar alternatives distributions. $\chi^{2}$ for minimally summed categories for adequate $N=6.028$ (df 8), p>.50. It would seem that the explanation of reduced response choice for superior identification from dissimilar alternatives has little basis in this analysis. The 3 dissimilar letter alternatives with significantly uneven error distributions in Experiment $I$ are in Experiment II more evenly distributed: $1,1,0 ; 3,3,1 ; 4,2,1(N=8$ and 12 respectively)

SUMMARY

1. When Ss are prepared for a brief stimulus exposure by seeing alternatives before the stimulus, selection of the target stimulus from the alternatives shown again after the stimulus exposure is very much more accurate than without such preparation.

2. Set improves accuracy for CVCs more than for letters and other stimuli used. It is suggested that CVCs are complex stimuli.

3. The similarity effect is pronounced, and it would seem 
that the effect is one of discrimination difficulty. Analysis of incorrect choices suggests that response selections were four-way choices for both similar and dissimilar alternatives.

4. Ss seeing alternatives before the stimulus slightly improve their identification scores in the latter half of each stimulus series. 
The first 4 stimulus series of Experiment $I$ and the first half of the 4 stimulus series of Experiment II are common. Experiment I Ss see 2 more types of stimuli; Experiment II Ss see more stimuli of the 4 common series. The After sequences of both experiments are identical in procedure; the difference in Before sequences is the second presentation of alternatives in Experiment II.

The analyses for separate experiments show significant effects for the following:

$\begin{array}{ll}\text { For Experiment I: } & \text { Exposure } \\ & \text { Stimulus } \\ & \text { Similarity } \\ & \text { Sequence } x \text { Exposure } \\ & \text { Exposure } x \text { Similarity }\end{array}$

For Experiment II: Sequence Similarity Sequence $\mathrm{x}$ stimulus

For Experiment II, Exposure is not significant (although p (.10), the reduced sample of stimulus types do not differ, and there is no suggestion of a sequence $x$ exposure interaction or an exposure $\mathrm{x}$ similarity interaction. In Experiment II the sequence and similarity effects have swept up most of the variance due to experimental effects. The significant sequence $\mathrm{x}$ stimulus interaction of Experiment II is not found in Experiment I, though the 4 common stimulus series in Experiment I do show some irregularity with different sequences. The sequence main effect is, of course, quite unique.

It might be informative to make a pseudo-experiment of responses to common stimuli and analyse the cell means 
simultaneously. The $n^{\prime}$ s are 4 and 6 ; the harmonic mean (4.80) is used in computations. Errors were very homogeneous.

TABLE 5. Experiments I and II: Analysis of Variance. $\begin{array}{llll}\text { Source } & \text { df } & \text { MS }\end{array}$

\begin{tabular}{|c|c|c|c|}
\hline Between Ss & 39 & & \\
\hline $\begin{array}{l}\text { A ( Sequence) } \\
\text { B (Exposure) } \\
\text { C (Experiments) } \\
A B \\
A C \\
B C \\
A B C \\
S \text { s/Groups }\end{array}$ & $\begin{array}{r}1 \\
1 \\
1 \\
1 \\
1 \\
1 \\
1 \\
32\end{array}$ & $\begin{array}{r}14.1840 \\
20.6323 \\
21.8899 \\
6.8918 \\
6.4195 \\
3.5851 \\
4.6978 \\
1.7979\end{array}$ & $\begin{array}{l}7.89 * * \\
11.48 * * \\
12.18 * * \\
3.83 \\
3.57 \\
1.99 \\
2.61\end{array}$ \\
\hline Within Ss & 280 & & \\
\hline $\begin{array}{l}\text { D (stimulus) } \\
\text { AD } \\
\text { BD } \\
\text { CD } \\
\text { ABD } \\
\text { ACD } \\
\text { BCD } \\
\text { ABCD } \\
D \times \text { Ss/Groups }\end{array}$ & $\begin{array}{l}3 \\
3 \\
3 \\
3 \\
3 \\
3 \\
3 \\
3 \\
96\end{array}$ & $\begin{array}{r}4.4424 \\
.1131 \\
.5613 \\
1.0122 \\
1.1376 \\
7.3357 \\
.2742 \\
.5000 \\
1.4168\end{array}$ & $3.14 *$ \\
\hline $\begin{array}{l}\mathrm{E} \text { (Similarity) } \\
\mathrm{AE} \\
\mathrm{BE} \\
\mathrm{CE} \\
\mathrm{ABE} \\
\mathrm{ACE} \\
\mathrm{BCE} \\
\mathrm{ABCE} \\
\mathrm{E} \times \mathrm{Ss} / \text { Groups }\end{array}$ & $\begin{array}{r}1 \\
1 \\
1 \\
1 \\
1 \\
1 \\
1 \\
1 \\
32\end{array}$ & $\begin{array}{r}13.4995 \\
.0422 \\
5.7418 \\
.0624 \\
3.9422 \\
1.0541 \\
2.4802 \\
.2294 \\
.7406\end{array}$ & $\begin{array}{l}18 \cdot 23 * * * \\
7 \cdot 75 * * \\
5.32 * \\
1.42 \\
3.35\end{array}$ \\
\hline $\begin{array}{l}D E \\
A D E \\
B D E \\
C D E \\
A B D E \\
A C D E \\
B C D E \\
A B C D E \\
D E \times \text { Ss/Groups }\end{array}$ & $\begin{array}{r}3 \\
3 \\
3 \\
3 \\
3 \\
3 \\
3 \\
3 \\
96\end{array}$ & $\begin{array}{r}.3317 \\
2.5149 \\
2.9883 \\
.1101 \\
2.0411 \\
2.1427 \\
.0434 \\
3.9789 \\
1.5557\end{array}$ & $\begin{array}{l}1.62 \\
1 \cdot 92 \\
1 \cdot 31 \\
1 \cdot 38 \\
2 \cdot 56\end{array}$ \\
\hline Total & 319 & & \\
\hline
\end{tabular}


After sequences for the two experiments are not equivalent. Cell mean totals are, for Experiment I: 27.25, for Experiment II: 31.166. The difference helps to create a significant experiments main effect and to reduce the sequence $x$ experiments interaction $(p<.10)$.

TABLE 6. Experiments I and II: Cell Mean Totals.

\begin{tabular}{crrr} 
Sequence & Exposure & Expt. I & Expt. II \\
\hline \multirow{3}{*}{ Before } & short & 14.00 & 20.334 \\
& Iong & 15.50 & 22.334 \\
\cline { 2 - 4 } & total & 29.50 & 42.668 \\
\hline \multirow{3}{*}{ After } & short & 8.50 & 14.165 \\
\cline { 2 - 4 } & long & 18.75 & 17.001 \\
\hline \hline
\end{tabular}

Table 6 of cell mean totals shows. the Experiment I After sequence short exposure total is much lower than the Experiment II counterpart, and less than chance (10.00). Exposure differences are taken up by the main effect in this combined analysis, and the sequence $\mathrm{x}$ experiments, exposure $\mathrm{x}$ experiments and sequence $\mathrm{x}$ exposure $\mathrm{x}$ experiments are all not significant $(p<.10,>.10$ and $>.10$ respectively).

The stimulus effect is significant, and so is the sequence $\mathrm{x}$ experiments $\mathrm{x}$ stimulus interaction, reflecting consistency with the separate analyses (see Fig. 10). The first halves of Experiment II show greater stimulus variation than the combined halves.

Similarity and exposure $\mathrm{x}$ similarity are significant but a sequence $\mathrm{x}$ exposure $\mathrm{x}$ similarity interaction appears rather than exposure $\mathrm{x}$ experiments $\mathrm{x}$ similarity. This 
interaction is plotted in Fig. 11 and shows a rather unexpected decline (from Experiment I) for Before sequence accuracy at long exposures when selecting responses from dissimilar alternatives.

The sequence $\mathrm{x}$ experiments $\mathrm{x}$ stimulus interaction appears to show forgetting in Experiment $I$ and set in Experiment II. Before sequences of Experiments I and II suggest that forgetting has taken place in Experiment I with all stimuli except letters-which is not unexpected, and tends to confirm the previous suggestions about forgetting of alternative location in Experiment I. After sequence comparisons are not easy to make. Scores show more variability than would be expected for largely comparable experimental conditions. CVCs are identified substantially better in Experiment I than in Experiment II.

Summary. The combined analysis has not really clarified the analysis of individual experiments, mainly because of low accuracy scores in After sequence, short exposure condition of Experiment $I$. We have some specific suggestions about forgetting in Before sequences that make good sense, but on the whole the lack of comparability between the After sequences prevents the expected interactions from appearing. 
Experiment III tests loss of information with delay of alternatives presentation. Only After sequences are used, with two delay intervals: $0.4 \mathrm{sec}$. and the $1.8 \mathrm{sec}$. used in Experiments $I$ and II.

Should there be loss of information during the $1.8 \mathrm{sec}$. delay, a sequence effect might plausibly implicate forgetting rather than set as Rubin argued against Kulpe and Bryan's experiment, and sperling (1960) more recently. We have already noted a marked forgetting of alternative location in the present stimulus identification experiments, and there is a sequence $\mathrm{x}$ exposure interaction suggesting both set and forgetting. Sperling's criticism refers to more complex responses than are at present used, although accuracy here is by no means perfect, and the marked similarity effect suggests the discriminative choice is by no means easy. The existence of a sequence effect shows that exposures are not low enough to make the stimulus destitute of information: Ss are able, in $\mathrm{E}^{\prime}$ 's reasoning, to pick up more relevant aspects of the stimulus when specifically prepared by prior alternatives. Decay of stimulus articulation when Ss are not prepared by prior alternatives, i.e. forgetting, may account for the sequence effect. Forgetting in this context does not refer to span reduction, or to alternative location, but to aspects of a foveally perceived unitary stimulus.

A difference between levels of interval will therefore be a test of short term forgetting. $0.4 \mathrm{sec}$. was chosen mainly because this was the shortest interval that was not annoying and it is also longer than intervals used in masking experiments (perhaps the two observations are related). 


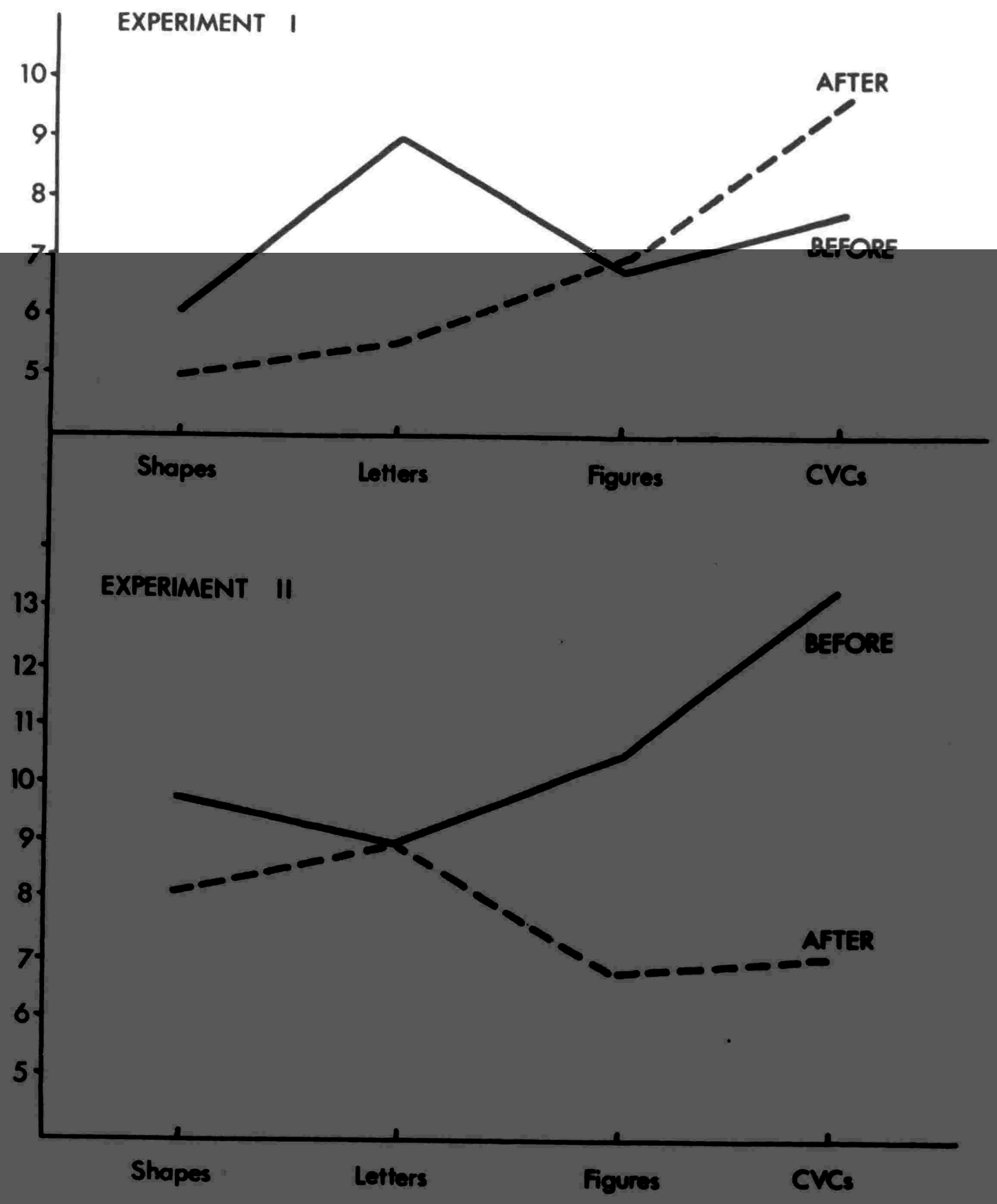

FIGURE 10. Experiments I and II: Sequence $x$ Experiments $x$ Stimulus interaction (based on responses to stimuli common to both experiments). 


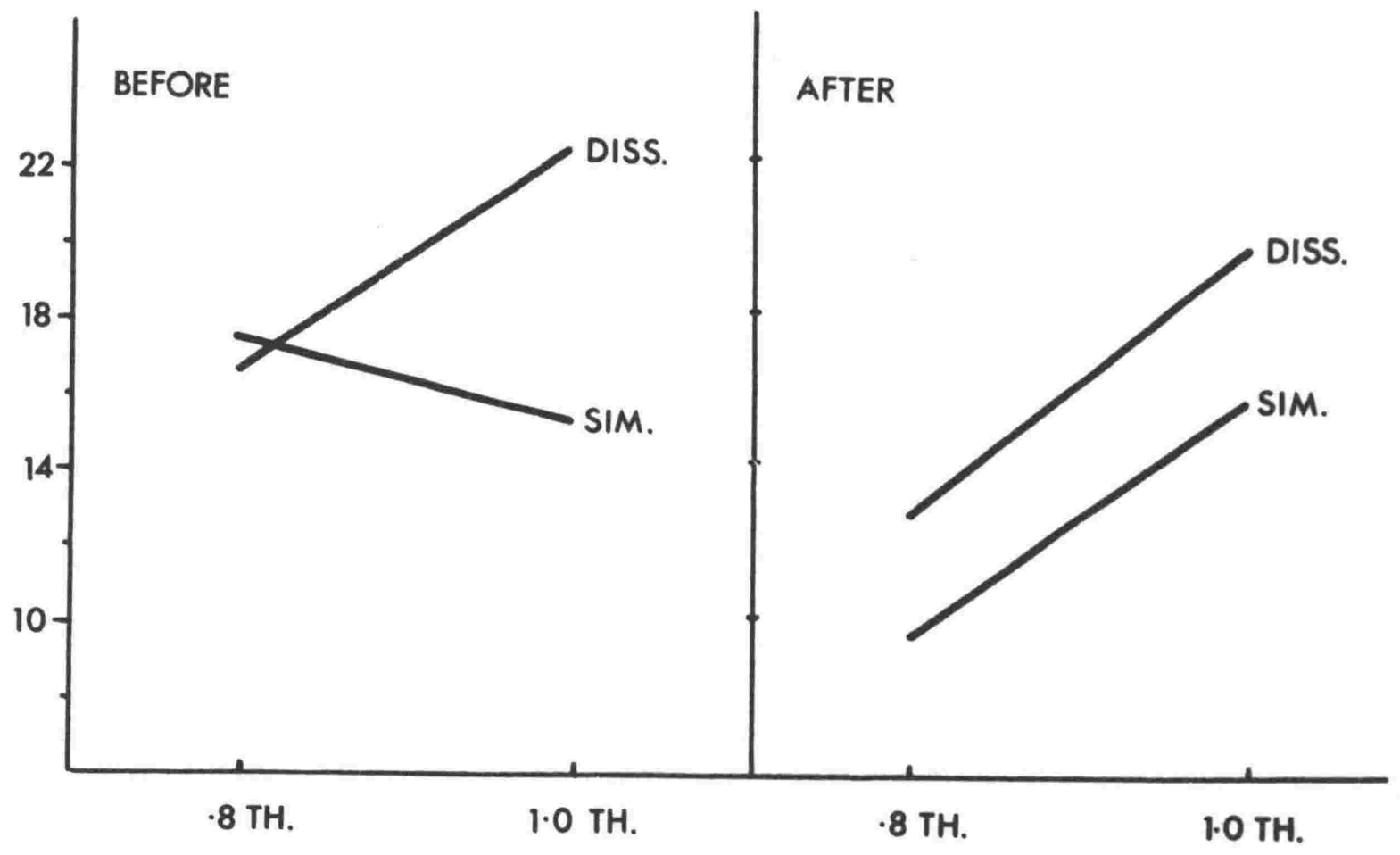

FIGURE 11. Experiments $I$ and II: Sequence $x$ Exposure $x$ Similarity interaction. 
METHOD

Stimuli. The latter halves of the 4 stimulus series (shapes, letters, figures and CVCs) of Experiment II were shown.

Subjects. $24 \mathrm{Ss}$ took part in this experiment: 14 from Experiment I, 8 from Experiment II (who had seen these stimuli before), and 2 new Ss. Ss were assigned randomly to the experimental conditions, after groups of low threshold (totals range $9.0 \mathrm{~ms}$ to $14.5 \mathrm{~ms}$ ) and high threshold (totals range $15.5 \mathrm{~ms}$ to $24.3 \mathrm{~ms}$ ) Ss were formed. Threshold groups receive different administrations of alternatives.

The design is for a five factor experiment: interval $\left(0.4,1.8\right.$ sec.), exposure $\left(.8,1.0\right.$ of $\mathrm{Ss}^{\prime}$ stimulus thresholds) and threshold (low or high subject threshold) are between s effects; stimulus and similarity are within s effects.

\section{RESULTS}

Error terms are homogeneous (Hartley's test) and within error terms are of the same order (Bartlett's test). Administrations of alternatives are confounded with the interval $\mathrm{x}$ threshold interaction, $\mathrm{F}=0.11$.

There are only two significant effects: threshold and similarity. High threshold ss identify the stimuli with significantly more accuracy when seeing the stimuli at exposures related to their high thresholds. The threshold effect would presumably disappear with adjustment for threshold covariance with accuracy. Similarity is significant. Exposure is not significant, nor do the stimulus series differ, though $\mathrm{p}<10$. 
TABLE 7. Experiment III: Analysis of Variance.

\begin{tabular}{|c|c|c|c|}
\hline Source & $d f$ & MS & $\mathrm{F}$ \\
\hline Between Ss & 23 & & \\
\hline $\begin{array}{l}\text { A (Interval) } \\
\text { B }(\text { Exposure) } \\
\text { C Threshold) } \\
\text { AB } \\
\text { AC } \\
\text { BC } \\
\text { ABC } \\
\text { Ss/Groups }\end{array}$ & $\begin{array}{r}1 \\
1 \\
1 \\
1 \\
1 \\
1 \\
1 \\
16\end{array}$ & $\begin{array}{r}2.2969 \\
3.2552 \\
7.1302 \\
.1302 \\
.1302 \\
.1302 \\
1.5054 \\
1.1510\end{array}$ & $\begin{array}{l}2.0 \\
2.83 \\
6.19 *\end{array}$ \\
\hline Within Ss & 168 & & \\
\hline $\begin{array}{l}D \text { (stimulus) } \\
\text { AD } \\
\text { BD } \\
\text { CD } \\
A B D \\
\text { ACD } \\
\text { BCD } \\
\text { ABCD } \\
D \times \text { Ss/Groups }\end{array}$ & $\begin{array}{l}3 \\
3 \\
3 \\
3 \\
3 \\
3 \\
3 \\
3 \\
48\end{array}$ & $\begin{array}{r}3.2691 \\
2.2691 \\
2.7830 \\
3.0435 \\
2.0746 \\
1.6858 \\
.3524 \\
.9218 \\
1.2899\end{array}$ & $\begin{array}{l}2.53 \\
1.76 \\
2.16 \\
2.36 \\
1.61 \\
1.31\end{array}$ \\
\hline E (similarity) & 1 & 20.6719 & $14 \cdot 38 \% *$ \\
\hline $\begin{array}{l}\mathrm{AE} \\
\mathrm{BE} \\
\mathrm{CE} \\
\mathrm{ABE} \\
\mathrm{ACE} \\
\mathrm{BCE} \\
\mathrm{ABCE} \\
\mathrm{E} \times \mathrm{Ss} / \text { Groups }\end{array}$ & $\begin{array}{r}1 \\
1 \\
1 \\
1 \\
1 \\
1 \\
1 \\
16\end{array}$ & $\begin{array}{r}.1302 \\
.8802 \\
.2552 \\
1.8802 \\
.0469 \\
3.2552 \\
.0050 \\
1.4375\end{array}$ & $\begin{array}{l}1.31 \\
2.26\end{array}$ \\
\hline $\begin{array}{l}\mathrm{DE} \\
\mathrm{ADE} \\
\mathrm{BDE} \\
\mathrm{CDE} \\
\mathrm{ABDE} \\
\mathrm{ACDE} \\
\mathrm{BCDE} \\
\mathrm{ABCDE}\end{array}$ & $\begin{array}{l}3 \\
3 \\
3 \\
3 \\
3 \\
3 \\
3 \\
3\end{array}$ & $\begin{array}{r}.3385 \\
.5469 \\
1.0746 \\
1.7830 \\
1.1370 \\
1.4357 \\
.5330 \\
2.6096\end{array}$ & $\begin{array}{l}1.07 \\
1.78 \\
1.14 \\
1.44 \\
2.61\end{array}$ \\
\hline $\mathrm{DE} \times \mathrm{Ss} /$ Groups & 48 & 1.0000 & \\
\hline Total & 191 & & \\
\hline
\end{tabular}

The effect of major interest, interval, is not significant, and there are no interval interactions. 
Identification of the stimulus is slightly better when alternatives are delayed for 1.8 seconds (see Table 8).

TABLE 8. Experiment III: Accuracy Totals.

\begin{tabular}{|c|c|c|c|c|c|}
\hline \multirow{2}{*}{$\begin{array}{l}\text { Subject } \\
\text { Threshold }\end{array}$} & \multirow[b]{2}{*}{ Exposure } & \multicolumn{2}{|c|}{ Short Interval } & \multicolumn{2}{|c|}{ Long Interval } \\
\hline & & sim. & diss. & sim. & diss. \\
\hline \multirow{3}{*}{ low } & short & 16 & 25 & 23 & 25 \\
\hline & long & 21 & 28 & 20 & 30 \\
\hline & total & 37 & 53 & 43 & 55 \\
\hline \multirow{3}{*}{ high } & short & 18 & 34 & 21 & 32 \\
\hline & long & 26 & 28 & 30 & 36 \\
\hline & total & 44 & 62 & 51 & 68 \\
\hline
\end{tabular}

The higher order interaction in this experiment ( $p .10)$ suggests irregularity in the data. If After sequences scores from Experiment II are used to supply data for the 1.8 second interval, the higher order interaction drops, and exposure $\mathrm{x}$ similarity and exposure $\mathrm{x}$ stimulus $\mathrm{x}$ similarity approach significance. There are no other changes.

Ss who had seen the stimuli before in Experiment II obtained a higher mean accuracy score (18.375) than those who had not (16.625), the two ss with experience of practice trials only scoring 16 and 17. The difference is due to two Ss from Experiment II. $F=1.44$ when tested against within $S$ variance $(F .05(1,168)=3.90)$.

\section{DISCUSSION}

The delay in this experiment refers to a second exposure in a tachistoscope. Consolidation of the stimulus 
trace, difficulty and time of decision are probably more important factors than forgetting over the brief delay intervals used. In many experiments delay refers to instructions given auditorily (Rubin's criticism of Kulpe, and Brown, sperling), or to instructions given from a discrete visual source (Chapman), or to interpolated responses (also Kulpe, and Kay \& Poulton, Murdock, etc.). of these and most other experiments on set and/or forgetting, Sperling used the shortest delay intervals-from 0.0 to 1.00 seconds, and his task was recording letter spans. The present task is simpler, though the stimulus information that $\mathrm{S}$ must carry is not negligible. Perhaps longer intervals are needed to show forgetting of single stimuli with the present conditions.

Shorter intervals cannot be used without danger of masking, or, if decision is presumed possible immediately on seeing alternatives, without avoiding increased accuracy because of information in after-images. If characteristic retinal after-stimulation is the major factor in short term visual storage when all information to be stored occurs in one brief exposure, then we are obliged to forego measuring information lasting less than 0.4 seconds with the current experimental set-up. While $\mathrm{E}$ agrees with the view that to demonstrate set it should be ensured that forgetting has not occurred when set is absent, a period when information decays which is coexistent with the period in which information is delivered from after-images cannot, in $\mathrm{E}^{\prime} \mathrm{s}$ opinion, be a period during which one can make a distinction between before and after stimulation. Naturally the entire S-R sequence is an organic one, and while interpolated recall, order of report, delay of instructions or withholding response information are all likely to affect response or memory measures, E regards response information given while stimulus information is still peripherally sensory as a condition not excluded by the term preparation. 
Perhaps we cannot say subjects are prepared instantaneously (Brown, 1960), although in certain ways Sperling implies the effect of his auditory instructions is immediate. Broadbent prefers to interpret Lawrence \& LaBerge's results to show selective perception appears "as the percept develops" (1958,p.224), a position in between Lawrence and co-workers' belief that set operates on memory and response factors and the traditional prior sensitisation. $E^{\prime}$ 's position is that the period in which after-images are articulated should be regarded as belonging very much to the stimulus side of the $S-R$ sequence, and even if preparation is not immediate, intervals of less than 0.4 seconds begin to shift into a limbo where the response information is neither before nor after stimulation, a period where short term memory may mean preciously little beyond retinal after-discharges.

The results of the present experiment give no suggestion that stimulus information decays over an interval of 1.8 seconds. This interval was tested against a 0.4 second interval which $\mathrm{E}$ would regard as the minimum period between two successive tachistoscopic presentations that allows full registration of the stimulus without masking. In fact, the slightly better accuracy with the longer interval may even suggest masking occurs at the shorter interval. Besides masking, $E$ does not at the moment wish to reject the speculation that a brief delay between stimulus and alternatives may assist $\mathrm{Ss}$ in decision or response processes: psychological refractory periods may not be irrelevant in the present context.

SUMMARY

1. When information necessary to identify a stimulus is delayed 0.4 and 1.8 seconds after the stimulus exposure, 
accuracy of identification is insignificantly better at the longer delay interval. Forgetting cannot be said to occur. Short term memory and after-images are discussed.

2. Two effects are significant: threshold (high threshold Ss score more than low threshold Ss) and similarity.

3. Differences between the 4 stimulus series are minor, and stimulus exposure is not significant in this experiment. 
S's task in this experiment is to rank the 4 alternatives in a likelihood order. Instead of checking only one alternative, $S$ is required to give this alternative location a 1 , being $S$ 's best judgment of which alternative was exposed as the stimulus, then a 2 for S's second choice, "in case your first choice is wrong" as S was told, and so on. E explained to $S$ that "sometimes you may have picked up something but you can't decide which alternative as it is. You might be able to narrow it down to two alternatives, but no further with any certainty. This experiment gives you a chance to get these second preferences in. If your second or even third choice is correct, it is scored with part marks." Ranked preferences allow scoring systems which may highlight the nature of response accuracy.

Experiments asking $S$ for second and third, etc., choices have been devised for many purposes, although the usual intention has been to show that $\mathrm{S}^{\prime} \mathrm{s}$ information is a continuous function of difficulty variables. Decision theorists (Tanner, 1955; Swets, Tanner \& Birasall, 1961) find that proportions of second choices correct are more than $1 / n-1$ in visual detection experiments, and that first choice accuracy is not affected by asking $\mathrm{S}$ for second choices. In an experiment not reported here, $\mathrm{E}$ found that if $\mathrm{Ss}$ are shown one of 5 CVCs at subliminal (limits) exposures, 1st, 2nd and 3rd choices are all above chance accuracy (.275 (.200), $.310(.250), .408$ (.333), respectively), and first choices are a little better when later choices are required than when they are not (.275 and .258 respectively). Comparable results are obtained when the task is a multiple choice test* on reading and lecture material for Psychology classes (when

* Kind thanks to K.D. White for administering this test. 
$\mathrm{z}$ for 2nd choices $=6.93,56 \mathrm{ss}, 4$ choices, 20 questions). Goldiamond (1961) noted that students giving preferences to such tests scored chance on second choices if they scored chance on first choices. Murdock (1954) found a similar type of result: later choices identifying nonsense syllables are correct above chance only for higher levels of illumination: if information is there, 2nd and later choices will show it. Murdock, Bricker \& Chapanis (1953) and many others find this usually unexpressed information to be of relevance in subception experiments, and Brown (1964) has used the technique to study all-or-none learning.

Subjects themselves sometimes feel all their information is not recorded by usual techniques (see Sperling's citations, 1960, p.1), even when the stimulus can be completely recorded (e.g. spans of letters). Titchener (1915) and Boring (1924) insisted that fuller descriptions than allowed by stereotyped judgments are necessary if we wish to study all the observer's information, and this is the point of many subception critics.

The present technique is one where S's responses exhaust stimulus information in a more efficient manner than enumerating numbers and types of stimulus elements. Stimulus matches are entirely restricted to 4 alternative responses and the rankings completely describe $S^{\prime}$ s stimulus information. The rank order can reliably measure nothing but accuracy and discriminable dissimilarity.

The above rationale is theoretical. In practice we cannot assume S will behave ideally. He is influenced by more factors than the central one of discriminating between matches of a briefly exposed stimulus. Experience of the stimulus material cannot very well be as even as experience of bursts of homogeneous illumination or of tones in noise. Nor, given $\mathrm{S}$ has some useful information, can we expect he 
will make perfectly rational decisions on this information. We can assume, however, that discrimination difficulty can be measured, and that sometimes incorrect choices are due to such difficulty. Ranking all alternatives should reduce the all-or-none nature of checking one alternative for a response, and errors due to difficult discrimination may become apparent with analysis of second choices.

METHOD

Two new series of letters and CVCs were prepared in the same way as before. $24 \mathrm{Ss}$ were randomly assigned to the experimental conditions with the restriction that no condition would have all high or all low threshold Ss (cut-off: $10.5 \mathrm{~ms}$ total of letter and CVC thresholds). All Ss except 2 had taken part in Experiment III, and all had participated in either Experiment I or II. The 2 who had not been Ss for Experiment III gave typical responses.

Interval is again tested in this experiment: delays of 0.4 and 1.8 seconds occur between stimulus and alternatives as in Experiment III. (The blank blue field between prior alternatives and the stimulus lasts 1.8 seconds). As in Experiment II, for Before sequences, alternatives are shown again after the stimulus exposure.

Experiment IV is a $2 \times 2 \times 2 \times 2 \times 2$ factorial experiment, $p$ (sequence), $q$ (exposure, .8, 1.0 of subjects' thresholds) and $r$ (interval) are between $s$ effects; s (stimulus) and $t$ (similarity) are within $s$ effects.

Scoring. Subjects' rankings were scored in 3 ways:

1. Only first choices considered: S's first choice scored either right or wrong, duplicating the earlier type of scoring, when S's single check mark is either right or wrong. 
2. Ranks: S's first choice is given a score of 3 , his second choice a score of 2 , and his third choice a score of 1. This scoring reduces the sharpness of the earlier scoring. S gets some credit for later correct choices.

3. First and second choices scored equally. S gets a score of 1 if his first or his second choice is correct. This scoring is taken to greatly reduce the importance of discriminating alternatives. If $\mathrm{S}$ has partly perceived the stimulus, this scoring does not penalise s's difficulty in selecting a correct response. It is not wholly erroneous to regard this scoring method as transforming a sometimes difficult 4-way choice into an easy 3-way choice (although this interpretation should not be taken literally-s still makes 4 responses to 4 alternatives).

Experiment IV, apart from Ss' responding, is much the same as Experiment II. Experiment II tests sequence, exposure, stimulus ( 4 types) and similarity, but only with the 1.8 second interval. Experiment III tests interval, exposure, stimulus ( 4 types) and similarity, but only for After sequences. Both Experiments II and III employ single responses to stimulus presentations. Experiment IV asks Ss to rank their responses, and sequence, exposure, interval, stimulus and similarity are tested. Only letters and CVCs are shown in this experiment. Subject thresholds, of no importance in Experiment II, but found to be significant in Experiment III, are here balanced within subject groups.

RESULTS

The data from the three scoring systems were analysed independently. Errors were homogeneous in every case (Hartley) and within errors $(p>.20,>.20,>.50$, Bartlett's test) were pooled to give adequate df for F ratios. Analyses of variance are given in the following three tables, 
and in Table 12 is given a comparison of $\mathrm{F}^{\prime} \mathrm{s}$ for all main effects and any interactions that were significant with any scoring system. The comparison table highlights what differences there are with the scoring systems.

A summary of each analysis is given here.

1. First choices analysis. $F^{\prime}$ s are significant for sequence, stimulus $(p=.05)$ and similarity main effects, and $\mathrm{BE}$ (exposure $\mathrm{x}$ similarity, $\mathrm{p}=.05$ ) and $\mathrm{BCD}$ (exposure $\mathrm{x}$ interval $x$ stimulus) interactions.

2. Ranked choices analysis, differential scoring for 1st, 2nd and 3rd choices. F's are significant for stimulus and similarity main effects, and $\mathrm{AD}$ (sequence $\mathrm{x}$ stimulus), $\mathrm{BE}$, $\mathrm{CD}$ (interval $\mathrm{x}$ stimulus) and $\mathrm{BCD}$ interactions.

3. First and second choices, both given equal scores. F's are significant for the stimulus effect, AD, BD (exposure $x$ stimulus), $\mathrm{BE}, \mathrm{BCD}$, and $\mathrm{BCDE}$ (exposure $\mathrm{x}$ interval $\mathrm{x}$ stimulus $\mathrm{x}$ similarity) interactions, but the similarity effect is absent.

The comparison table shows that consistent $F^{\prime}$ s exist for the exposure (NS), interval (NS), stimulus, exposure $x$ similarity and exposure $\mathrm{x}$ interval $\mathrm{x}$ stimulus effects, no matter which scoring system is used, although there is variation both above and below the significance level. On the whole, CVCs are judged more correctly than letters; selection from dissimilar alternatives is better than from similar alternatives only at long stimulus exposures; and CVCs are judged substantially better than letters only at long stimulus exposures and short delays between stimulus and alternatives presentations. These conclusions are more or less independent of the scoring system.

Comparing First Choices and Ranked Choices analyses. Noting changes that occur when scoring 2nd and 3 rd choices 
TABLE 9. Experiment IV: Analysis of Variance, First Choices.

\begin{tabular}{|c|c|c|c|}
\hline Source & $d f$ & MS & $F$ \\
\hline Between Ss & 23 & & \\
\hline $\begin{array}{l}\text { A }(\text { Sequence }) \\
\mathrm{B} \text { (Exposure } \\
\mathrm{C} \text { (Interval) } \\
\mathrm{AB} \\
\mathrm{AC} \\
\mathrm{BC} \\
\mathrm{ABC} \\
\mathrm{Ss} / \text { Groups }\end{array}$ & $\begin{array}{r}1 \\
1 \\
1 \\
1 \\
1 \\
1 \\
1 \\
16\end{array}$ & $\begin{array}{r}8.7604 \\
1.7604 \\
3.7604 \\
3.0104 \\
1.2604 \\
.5104 \\
.0105 \\
1.6146\end{array}$ & $\begin{array}{l}5.43 * \\
1.09 \\
2.33 \\
1.86\end{array}$ \\
\hline Within Ss & 72 & & \\
\hline $\begin{array}{l}\text { D (Stimulus) } \\
\text { AD } \\
\text { BD } \\
\text { CD } \\
\text { ABD } \\
\text { ACD } \\
\text { BCD } \\
\text { ABCD } \\
\text { Dx Ss/Groups }\end{array}$ & $\begin{array}{l}1 \\
1 \\
1 \\
1 \\
1 \\
1 \\
1 \\
1 \\
16\end{array}$ & $\begin{array}{r}5.5104 \\
3.0104 \\
1.7604 \\
4.5937 \\
1.7605 \\
.5105 \\
10.0105 \\
.0936 \\
1.0313\end{array}$ & $\begin{array}{l}4 \cdot 04 * \\
2 \cdot 21 \\
1 \cdot 29 \\
3 \cdot 37 \\
1 \cdot 29 \\
7 \cdot 34 * *\end{array}$ \\
\hline $\begin{array}{l}\mathrm{E} \text { (Similarity) } \\
\mathrm{AE} \\
\mathrm{BE} \\
\mathrm{CE} \\
\mathrm{ABE} \\
\mathrm{ACE} \\
\mathrm{BCE} \\
\mathrm{ABCE} \\
\mathrm{E} \times \mathrm{Ss} / \text { Groups }\end{array}$ & $\begin{array}{r}1 \\
1 \\
1 \\
1 \\
1 \\
1 \\
1 \\
1 \\
16\end{array}$ & $\begin{array}{r}14.2604 \\
.2604 \\
5.5104 \\
1.7604 \\
.0105 \\
.5105 \\
.0105 \\
1.2602 \\
1.7604\end{array}$ & $\begin{array}{c}10.46 * * \\
4.04 * \\
1.29\end{array}$ \\
\hline $\begin{array}{l}\mathrm{DE} \\
\mathrm{ADE} \\
\mathrm{BDE} \\
\mathrm{CDE} \\
\mathrm{ABDE} \\
\mathrm{ACDE} \\
\mathrm{BCDE} \\
\mathrm{ABCDE} \\
\mathrm{DE} \times \mathrm{Ss} / \text { Groups }\end{array}$ & $\begin{array}{r}1 \\
1 \\
1 \\
1 \\
1 \\
1 \\
1 \\
1 \\
16\end{array}$ & $\begin{array}{r}2.3437 \\
3.7605 \\
.2605 \\
.0105 \\
3.0102 \\
.0103 \\
.2603 \\
.2607 \\
1.3201\end{array}$ & $\begin{array}{l}1.72 \\
2.76\end{array}$ \\
\hline Total & 95 & & \\
\hline
\end{tabular}


TABLE 10. Experiment IV: Analysis of Variance, Ranked Choices.

\begin{tabular}{|c|c|c|c|}
\hline Source & $d f$ & MS & $F$ \\
\hline Between Ss & 23 & & \\
\hline $\begin{array}{l}\text { A( Sequence) } \\
\text { B Exposure) } \\
\text { C(Interval) } \\
\text { AB } \\
\mathrm{AC} \\
\mathrm{BC} \\
\mathrm{ABC} \\
\mathrm{Ss} / \text { Groups }\end{array}$ & $\begin{array}{r}1 \\
1 \\
1 \\
1 \\
1 \\
1 \\
1 \\
16\end{array}$ & $\begin{array}{r}24.0000 \\
.1666 \\
5.0416 \\
10.6667 \\
2.0417 \\
9.3751 \\
.0416 \\
8.3750\end{array}$ & $\begin{array}{l}2.87 \\
1.27 \\
1.12\end{array}$ \\
\hline Within Ss & 72 & & \\
\hline $\begin{array}{l}D(\text { stimulus }) \\
A D \\
B D \\
C D \\
A B D \\
A C D \\
B C D \\
A B C D \\
D \times \text { Ss/Groups }\end{array}$ & $\begin{array}{r}1 \\
1 \\
1 \\
1 \\
1 \\
1 \\
1 \\
1 \\
16\end{array}$ & $\begin{array}{r}51.0416 \\
26.0417 \\
9.3751 \\
24.0001 \\
7.0416 \\
10.6666 \\
42.6665 \\
1.6668 \\
3.8125\end{array}$ & $\begin{array}{l}9.12 * * \\
4.65 * \\
1.67 \\
4.29 \% \\
1.26 \\
1.91 \\
7.62 * *\end{array}$ \\
\hline $\begin{array}{l}\mathrm{E}(\text { Similarity) } \\
\mathrm{AE} \\
\mathrm{BE} \\
\mathrm{CE} \\
\mathrm{ABE} \\
\mathrm{ACE} \\
\mathrm{BCE} \\
\mathrm{ABCE} \\
\mathrm{E} \mathrm{X} \mathrm{Ss} / \text { Groups }\end{array}$ & $\begin{array}{r}1 \\
1 \\
1 \\
1 \\
1 \\
1 \\
1 \\
1 \\
16\end{array}$ & $\begin{array}{r}23.9997 \\
.6669 \\
42.6670 \\
2.0420 \\
.1664 \\
1.0414 \\
2.0413 \\
7.0420 \\
7.3958\end{array}$ & $\begin{array}{l}4.29 * \\
7.62 \% *\end{array}$ \\
\hline $\begin{array}{l}\mathrm{DE} \\
\mathrm{ADE} \\
\mathrm{BDE} \\
\mathrm{CDE} \\
\mathrm{ABDE} \\
\mathrm{ACDE} \\
\mathrm{BCDE} \\
\mathrm{ABCDE} \\
\mathrm{DE} \times \mathrm{Ss} / \text { Groups }\end{array}$ & $\begin{array}{r}1 \\
1 \\
1 \\
1 \\
1 \\
1 \\
1 \\
1 \\
16\end{array}$ & $\begin{array}{r}7.0420 \\
26.0414 \\
9.3749 \\
.0000 \\
3.3753 \\
.0000 \\
8.1665 \\
16.6665 \\
5.5833\end{array}$ & $\begin{array}{l}1.26 \\
4.65 * \\
1.67\end{array}$ \\
\hline Total & 95 & & \\
\hline
\end{tabular}


TABLE 11. Experiment IV: Analysis of Variance, First and Second Choices.

\begin{tabular}{|c|c|c|c|}
\hline Source & $d f$ & MS & $\mathrm{F}$ \\
\hline Between Ss & 23 & & \\
\hline $\begin{array}{l}\mathrm{A}(\text { Sequence }) \\
\mathrm{B}(\text { Exposure } \\
\mathrm{C}(\text { Interval) } \\
\mathrm{AB} \\
\mathrm{AC} \\
\mathrm{BC} \\
\mathrm{ABC} \\
\mathrm{Ss} / \text { Groups }\end{array}$ & $\begin{array}{r}1 \\
1 \\
1 \\
1 \\
1 \\
1 \\
1 \\
16\end{array}$ & $\begin{array}{r}2.3438 \\
.5104 \\
.0104 \\
.5104 \\
.0104 \\
3.0105 \\
.0937 \\
1.4063\end{array}$ & 1.67 \\
\hline Within Ss & 72 & & \\
\hline $\begin{array}{l}\text { D (stimulus) } \\
\text { AD } \\
\text { BD } \\
\text { CD } \\
\text { ABD } \\
\text { ACD } \\
\text { BCD } \\
\text { D x Ss/Groups }\end{array}$ & $\begin{array}{r}1 \\
1 \\
1 \\
1 \\
1 \\
1 \\
1 \\
16\end{array}$ & $\begin{array}{r}6.5104 \\
4.5937 \\
3.7605 \\
3.0105 \\
1.7604 \\
.8437 \\
5.5102 \\
.5104\end{array}$ & $\begin{array}{l}7.41 * \% \\
5.23 * \\
4.28 * \\
3.43 \\
2.00 \\
6.27 *\end{array}$ \\
\hline $\begin{array}{l}\mathrm{E} \text { (Similarity) } \\
\mathrm{AE} \\
\mathrm{BE} \\
\mathrm{CE} \\
\mathrm{ABE} \\
\mathrm{ACE} \\
\mathrm{BCE} \\
\mathrm{ABCE} \\
\mathrm{E} \times \mathrm{Ss} / \text { Groups }\end{array}$ & $\begin{array}{r}1 \\
1 \\
1 \\
1 \\
1 \\
1 \\
1 \\
1 \\
16\end{array}$ & $\begin{array}{r}1.2604 \\
.0104 \\
6.5105 \\
.8438 \\
.0104 \\
.0104 \\
.5103 \\
.2604 \\
.9896\end{array}$ & $\begin{array}{l}1.43 \\
7 \cdot 41 * *\end{array}$ \\
\hline $\begin{array}{l}\mathrm{DE} \\
\mathrm{ADE} \\
\mathrm{BDE} \\
\mathrm{CDE} \\
\mathrm{ABDE} \\
\mathrm{ACDE} \\
\mathrm{BCDE} \\
\mathrm{ABCDE} \\
\mathrm{DE} \times \mathrm{Ss} / \text { Groups }\end{array}$ & $\begin{array}{r}1 \\
1 \\
1 \\
1 \\
1 \\
1 \\
1 \\
1 \\
16\end{array}$ & $\begin{array}{l}.0938 \\
.8438 \\
.8436 \\
.0936 \\
2.4348 \\
.0938 \\
3.7607 \\
2.9193 \\
1.1354\end{array}$ & $\begin{array}{l}2.67 \\
4.28 * \\
3.32\end{array}$ \\
\hline Total & 95 & & \\
\hline
\end{tabular}


proportionately, it can be seen that the sequence main effect drops, but a new sequence $\mathrm{x}$ stimulus effect and a new sequence $x$ stimulus $x$ similarity effect have appeared. The interval $x$ stimulus interaction of the first choices analysis ( $p<$ 10) now exceeds the .05 level. Since the between error has increased and the within error decreased (percentage-wise), it is doubtful that these changes can be considered outstanding.

TABLE 12. Experiment IV: Comparison of $F$ Ratios for the Different Scoring systems.

Source
A (Sequence)
B (Exposure)
C (Interval)
D (Stimulus)
E (Similarity)

$\mathrm{AD}$

$\mathrm{BD}$

$\mathrm{BE}$

CD

$\mathrm{ADE}$

BCD

$\mathrm{BCDE}$
First Choices
Ranked Choices 1st and 2nd Choices 
now $p>.20$ when second correct choices are given equal weight. However, the exposure $\mathrm{x}$ interval $\mathrm{x}$ stimulus $\mathrm{x}$ similarity interaction is now significant, and the exposure $\mathrm{x}$ similarity interaction has increased.

As well, sequence $x$ stimulus (cf. ranked choices) and exposure $x$ stimulus interactions have appeared and their size is somewhat more than might be accounted for by error reduction. Inspection of subtotal figures shows that there is little to choose between a first choice sequence effect and a sequence $x$ stimulus effect with first and second choices, the sequence effect being obvious only for CVCs in both cases. The exposure $\mathrm{x}$ stimulus subtotals show a similar change: in both of these types of scoring analyses the exposure difference is more pronounced for CVCs.

These concomitant changes and irregularities make it difficult to come to a clear decision on the reduction of the similarity effect when first and second choices are scored equally. If totals figures are compared, the similar and dissimilar totals for first choices are 97 and 134, for first and second choices 160 and 171. That is, selection from similar alternatives benefits more when second choices are considered. However, there is a little less opportunity for dissimilar second choices to increase, and the $z$ from an independent proportions test ( 2 nd choice $N$ = Total $N$ - 1st choices correct) is only 1.458. In summary, E would take the hypothesis that the lack of similarity effect in scores from first and second choices may only be partly true.

Note on Single and Several Choices. $231 / 480$ first choices were correct. In Experiment II, which
had largely comparable perceptual conditions, $239 / 480$ choices were correct in first halves, and 251/480 in second halves (letters and CVCs, as in this experiment). If anything, asking Ss for several choices may depress accuracy slightly. 
Forgetting. The interval main effect is not significant, but the exposure $\mathrm{x}$ interval $\mathrm{x}$ stimulus interaction is significant, regardless of which scoring system is employed. Interval $x$ stimulus subtotals indicate no stimulus difference at long delays of alternatives, but less accuracy of letters at short delays of alternatives. The interaction for these subtotals is not significant ( $p<.10$ for first choice analysis). The subtotals for the significant exposure $\mathrm{x}$ interval $\mathrm{x}$ stimulus interaction (see Fig. 13) show that some forgetting of CVCs may have occurred at long exposures over the period between 0.4 and 1.8 seconds intervals of stimulus and alternatives, but the accuracy of letter identification over the same time delay, and also at long exposures, has actually increased. In addition, accuracy for both letters and CVCs increases as delay increases when stimuli are shown at short exposures. The "evidence" for forgetting applies to CVCs shown at long exposures, other combinations showing that increased delay facilitates accuracy. This may yet be meaningful, and interval will be tested in the next experiment.

Administrations of alternatives were confounded with exposure levels in this experiment, and exposure is not significant with any scoring system. Should the alternatives administered to $\mathrm{Ss}$ in long exposure conditions offer, on the whole, more difficult selections than alternatives administered to $\mathrm{Ss}$ in short exposure conditions, then the absence of an exposure main effect is explained by the confounding. The exposure $\mathrm{x}$ similarity interaction is significant, and that this might be due to an absence of any difference betwen similar and dissimilar alternatives for only one administration E regards as unlikely. This interaction has the same pattern of subtotals in this experiment (longer exposures mainly help selection from dissimilar alternatives) as it has in Experiment $I$ and the 


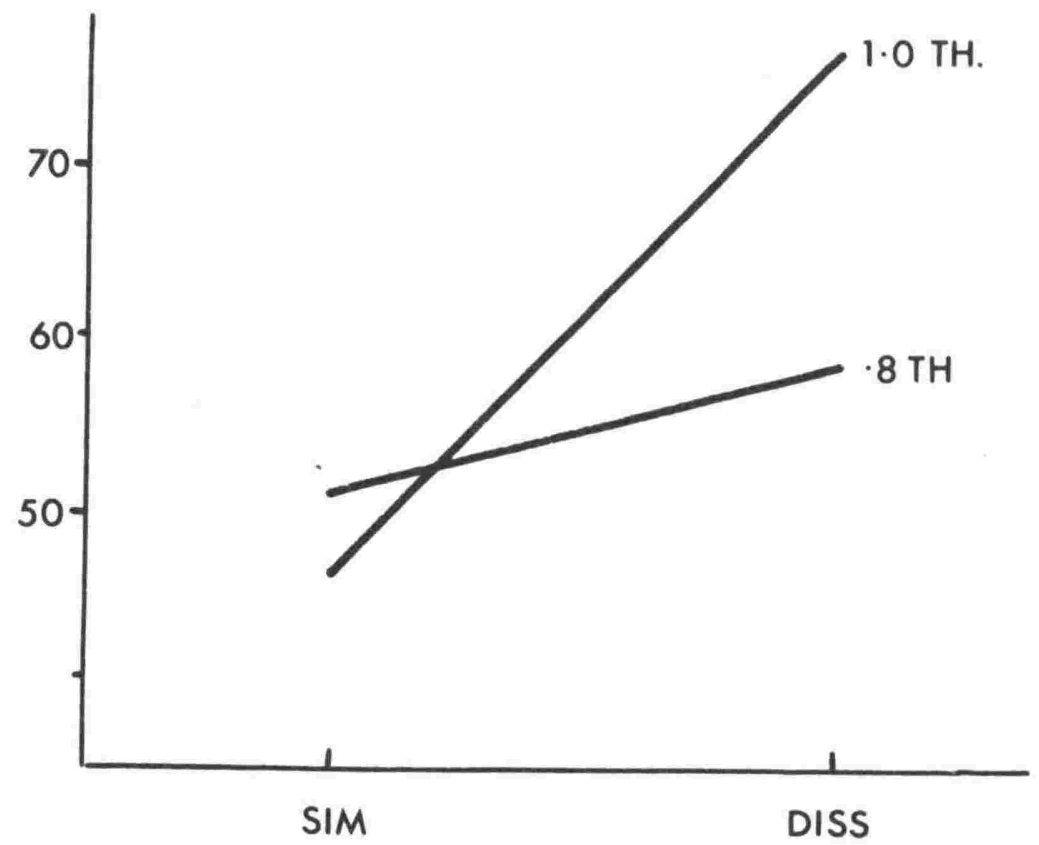

FIGURE 12. Experiment IV: Exposure x Similarity interaction (First Choices).

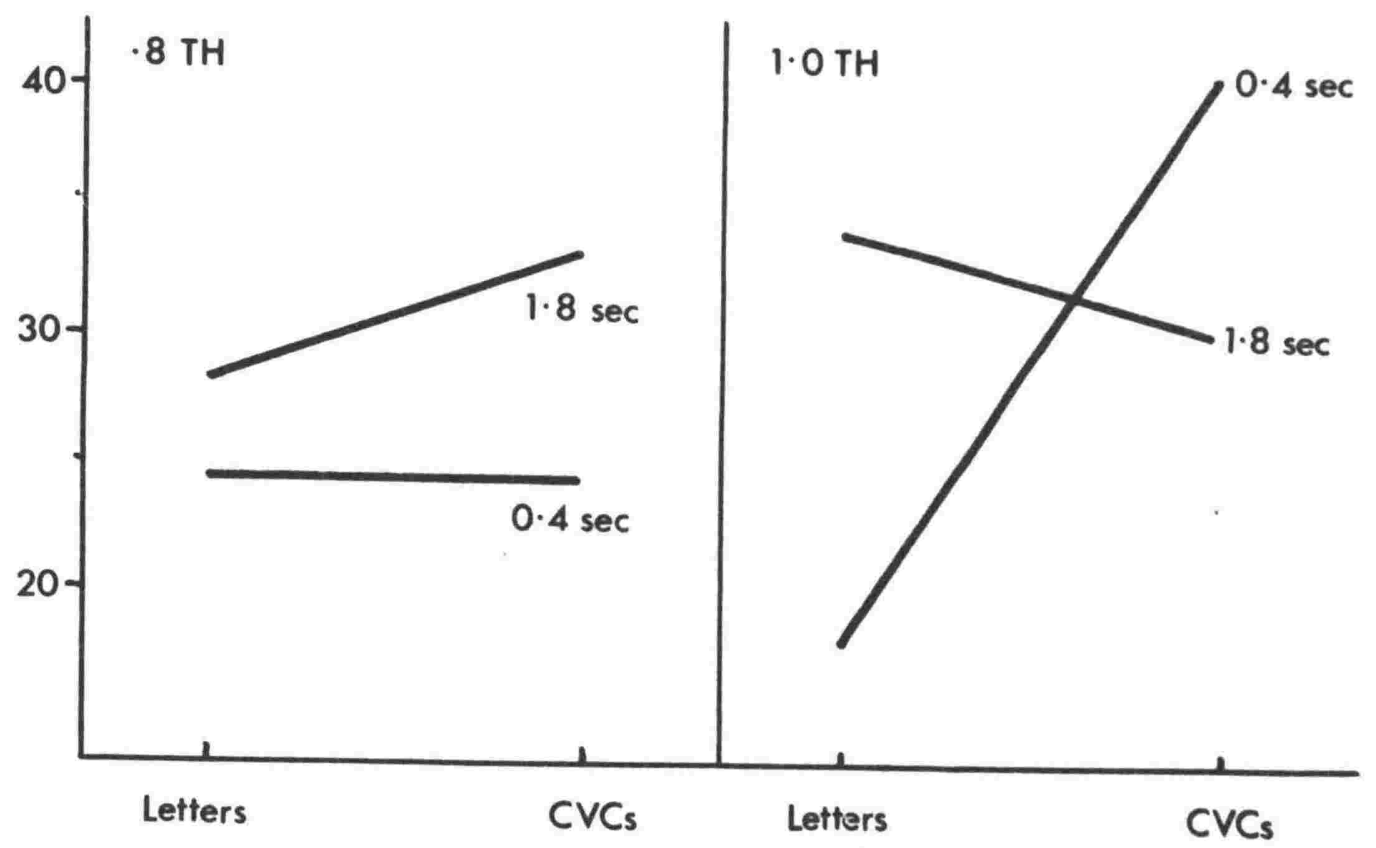

FIGURE 13. Experiment IV: Exposure $\mathrm{x}$ Interval $\mathrm{x}$ Stimulus interaction (First Choices). 
suggestion of this interaction $(p<.10)$ in Experiment II. This does not eliminate the possibility of administration differences, but argues against them. Exposure is not significant in Experiment II or III.

\section{SUMMARY}

1. When Ss rank their identification responses 1 to 4 in a likelihood order, the overall accuracy of first choices is much the same as when Ss make only one identification response, as in other experiments.

2. When equal weight is given to first and second choices, a scoring system which minimises the difficulty of discriminiating alternatives, the similarity effect strongly evident for first choices now disappears. More second choices from similar alternatives are correct than from dissimilar alternatives, although when proportions of totals are tested, the critical ratio for these second choices is 1.458. Alterations in interactions prevent unequivocal assertion of the effect of scoring second choices equally, but there is still nothing to contradict the hypothesis that similarity reflects discrimination difficulty rather than anything else.

3. Exposure differences occur mainly when selections from dissimilar alternatives are made, as has been found in earlier experiments.

4. The main effect of interval is not significant but the suggestion ( $p<.10$ first choices, <.05 ranked choices) of an interval $x$ stimulus interaction shows that letters are less well identified at short delays. This complicates the interpretation of the significant interval $\mathrm{x}$ exposure $\mathrm{x}$ stimulus interaction: forgetting of CVCs appears to occur 
at long delays and long exposures, but at short exposures both letters and CVCs are identified better at long delays.

5. Set (Before and After vs. only After) is apparent, but not as clearly as in other experiments. 
Experiment $\mathrm{V}$ again tests forgetting of the stimulus by comparing delays of 1.8 and 3.0 seconds interval between the stimulus exposure and presentation of alternatives. The long 3.0 seconds delay was chosen since in Experiment IV delays of up to 1.8 seconds appear to have irregular effects. Forgetting in the longer interval in this experiment will almost certainly be related to a more memorial type of forgetting rather than after-image decadence.

Subjects rank their choices and the three different analyses of Experiment IV are again undertaken. Set is once more defined as the comparison between alternatives seen before and after the stimulus and alternatives seen only after the stimulus.

Stimuli were letters and CVCs, 8 series of each. Six of these were used in earlier experiments, and two more were prepared, the CVCs this time from Noble's (1961) list (association values between .10 and .40)as Archer's list was not available when required.

For this experiment, the fixation spot in the centre of the blank field was replaced with 2 thin vertical lines $\frac{1}{4} "$ long, $\frac{3}{4} "$ above and $\frac{3}{4} "$ below the central point. The centre of the stimuli flash midway between the lines.

The levels of exposure were .7 and 1.0 of Ss' stimulus thresholds, the shorter exposure chosen to yield a clearer threshold effect than in some earlier experiments. The "thresholds" for CVCs were altered: if S's CVC threshold was not $.5 \mathrm{~ms}$ lower than his letter threshold, E adjusted the value to make it so. E reasoned that by removing what masking effect the fixation spot had, CVCs should be easier 
to pick up than letters; there are two more letters for inferential identification, and configuration cues probably become stronger. The intention was to eliminate a stimulus effect.

The experiment is a complete $2 \times 2 \times 2 \times 2 \times 2$ factorial design, with $8 \mathrm{ss}$ to receive all conditions. All estimates are thus within $\mathrm{S}$ and tests will perhaps be more precise because of this. The $8 \mathrm{Ss}$ used had taken part in Experiments III and IV, 7 had been Ss for Experiment I, the other one had been a subject for Experiment II. They are well practised Ss and since all took part in Experiment IV are familiar with ranking choices.

The 8 series of stimuli were balanced over the 8 Sequence $x$ Exposure $x$ Interval combinations by means of a cyclic latin square with the $8 \mathrm{ss}$ randomly assigned to rows of treatment-series combinations. One sequence $\mathrm{x}$ exposure $\mathrm{x}$ interval combination is given per session, which lasts 10 to 12 minutes. The sessions were spread over a fortnight, though some $\mathrm{Ss}$ had two sessions in one day. There were no practice trials for this experiment: one test stimulus is shown and the two practice stimuli at the beginning of each letter and CVC series ( $S$ does not know they are practice stimuli). In each session letters are shown first. Order of giving sessions was partly randomised: E would warm up the tachistoscope in readiness for a treatment combination that had fewest data entries, and then inquire after Ss who might have time to spare. Where possible, E tried to alternate sequence in session orders, but apart from this, there is no discernable pattern in the ordering of sessions. $\mathrm{E}$ introduced each session with either "This is one where you control the stimulus"(After condition), or "This is one where I start the sequence" (Before and After condition). otherwise, instructions were the same as before. 
All Ss see all stimuli twice: each half series of stimuli is seen once with similar alternatives and again a week later with dissimilar alternatives, or vice versa. The repeated half series is given in the same interval, balanced over other conditions for both groups of $\mathrm{Ss}$.

\section{RESULTS}

For the analysis of first choices subject interactions were homogeneous (Bartlett) and were pooled. Subject interactions were also homogeneous for the analysis of first and second choices scored equally, but were not homogeneous for the analysis of ranked choices $(p \doteq .025)$. These terms were nevertheless pooled to allow comparing $\mathrm{F}^{\prime} \mathrm{s}$, and all subject interactions have been tabled for the reader's observation. Df's for $\mathrm{F}$ numerators are all 1 , and for subject interactions all.7; pooled error df is 217 .

Changes in $\mathrm{F}$ ratios when specific error terms are used are for the most part minor ones. For first choices, the stimulus and BCDE effects decrease ( $B C D E$ because of reduced $\mathrm{df})$ and $\mathrm{DE}$, the stimulus $\mathrm{x}$ similarity interaction, is significant. Exposure also shows a slight decrease, and similarity a slight increase. For ranked choices there are no significance changes, although exposure and similarity effects have decreased, ACD increased and DE, ABCD and BCDE are all $\mathrm{p}<.10$. For the analysis giving equal weight to first and second choices, the stimulus effect is now not significant, and $A E$ and $D E$ are now $p<.10$.

The results from pooled error terms will be discussed. Sequence and exposure are significant main effects $(p<.001)$, and interval is not, for all ways of scoring Ss' choices. The stimulus and similarity effects are not pronounced with any scoring system, though similarity is significant in the 
TABLE 13. Experiment V: Analysis of Variance, First Choices.

Source

MS

Subject F Interaction

\section{A (Sequence) \\ B (Exposure) \\ C (Interval) \\ D (Stimulus) \\ E (Similarity)}

$A B$

$A C$

$A D$

$\mathrm{AE}$

BC

BD

$\mathrm{BE}$

CD

$\mathrm{CE}$

$\mathrm{DE}$

$A B C$

$A B D$

$\mathrm{ABE}$

$\mathrm{ACD}$

$\mathrm{ACE}$

$\mathrm{ADE}$

$\mathrm{BCD}$

$\mathrm{BCE}$

$\mathrm{BDE}$

$\mathrm{CDE}$

$A B C D$

$\mathrm{ABCE}$

$A B D E$

ACDE

$\mathrm{BCDE}$

ABCDE
144.0000

22.5625

2.2500

5.6407

5.0625

1.2657

.7657

.0625

.0157

.0157

.0625

.3907

1.0000

.0157

3.0625

.9998

.0155

.0623

1.8905

2.2498

1.8905

1.2655

.0623

.3905

.7655

1.5628

.3910

2.2503

.0628

5.0628

.1401
1.1071

2.8660

2.7857

1.4799

.6160

1.6764

2.5871

.7590

1.0335

1.6406

1.0268

1.1764

.5715

.7835

.4911

$2 \cdot 38$

1.5179

.7299

.7768

1.1406

11964

2.4263

.8906

1.8839

.8370

1.9620

1.1019

1.5349

.6250

1.2589

.9196

.6191
$112.04 * * *$

$17.56 \% * *$

1.75

4.39*

$3.94 \%$

Note. Mean squares are given for subject interactions. Pooled error is used for $F$ tests $(1,217 \mathrm{df})$. 
TABLE 14. Experiment V: Analysis of Variance, Ranked Choices.

Source

MS

Subject

F Interaction

\begin{tabular}{|c|c|c|c|}
\hline 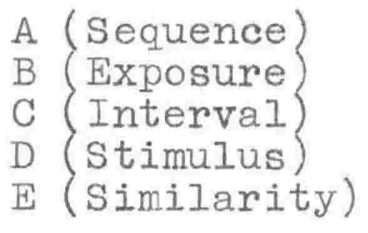 & $\begin{array}{r}600.2500 \\
121.0000 \\
2.6407 \\
19.1407 \\
3.5157\end{array}$ & $\begin{array}{r}3.8482 \\
13.7589 \\
18.1495 \\
10.2388 \\
4.7210\end{array}$ & $\begin{array}{c}105.44 * * * \\
21.25 * * * \\
3.36\end{array}$ \\
\hline $\begin{array}{l}\mathrm{AB} \\
\mathrm{AC} \\
\mathrm{AD} \\
\mathrm{AE} \\
\mathrm{BC} \\
\mathrm{BD} \\
\mathrm{BE} \\
\mathrm{CD} \\
\mathrm{CE} \\
\mathrm{DE}\end{array}$ & $\begin{array}{r}.1407 \\
6.2500 \\
.0625 \\
1.5625 \\
1.0000 \\
3.0625 \\
7.5625 \\
1.2655 \\
.0155 \\
8.2655\end{array}$ & $\begin{array}{l}5.9174 \\
7.6339 \\
4.5357 \\
4.3124 \\
5.1518 \\
5.4107 \\
8.2679 \\
3.0781 \\
.3281 \\
2.1674\end{array}$ & $\begin{array}{l}1.33 \\
1.45\end{array}$ \\
\hline $\begin{array}{l}A B C \\
A B D \\
A B E \\
A C D \\
A C E \\
A D E \\
B C D \\
B C E \\
B D E \\
C D E\end{array}$ & $\begin{array}{r}5.6405 \\
.3905 \\
3.5155 \\
33.0625 \\
5.0625 \\
3.0625 \\
5.0625 \\
1.5625 \\
.5625 \\
4.5158\end{array}$ & $\begin{array}{r}6.8817 \\
5.8996 \\
6.2031 \\
2.3214 \\
2.7143 \\
1.8750 \\
10.6607 \\
9.9821 \\
4.6786 \\
6.7388\end{array}$ & $5.81 *$ \\
\hline $\begin{array}{l}A B C D \\
A B C E \\
A B D E \\
A C D E \\
B C D E\end{array}$ & $\begin{array}{r}15.0158 \\
5.6408 \\
1.8908 \\
1.5625 \\
10.5625\end{array}$ & $\begin{array}{l}2.9888 \\
1.8281 \\
3.3460 \\
4.4821 \\
2.0357\end{array}$ & 1.86 \\
\hline$A B C D E$ & .1404 & 6.3103 & \\
\hline
\end{tabular}

Note. Mean squares are given for subject interactions. Pooled error is used for $F$ tests $(1,217 \mathrm{df})$. 
TABLE 15. Experiment V: Analysis of Variance, First and Second Choices.

Source MS

Subject F Interaction

\begin{tabular}{|c|c|c|c|}
\hline $\begin{array}{l}\text { A (Sequence) } \\
\text { B (Exposure) } \\
\text { C (Interval) } \\
\text { D (stimulus) } \\
\text { E (Similarity) }\end{array}$ & $\begin{array}{r}62.0156 \\
11.2906 \\
2.6406 \\
4.0000 \\
.0156\end{array}$ & $\begin{array}{r}.4888 \\
1.8817 \\
1.4174 \\
1.2589 \\
.7388\end{array}$ & $\begin{array}{l}73.01 * * * \\
13.41 * * * \\
3.11 \\
4.71 \%\end{array}$ \\
\hline $\begin{array}{l}A B \\
A C \\
A D \\
A E \\
B C \\
B D \\
B E \\
C D \\
C E \\
D E\end{array}$ & $\begin{array}{r}.2501 \\
.5626 \\
.1407 \\
2.2501 \\
.0626 \\
1.2657 \\
1.0001 \\
.7657 \\
.5626 \\
.7657\end{array}$ & $\begin{array}{r}.4375 \\
.9286 \\
.4888 \\
.5625 \\
1.3036 \\
1.1304 \\
.8303 \\
.9531 \\
.6438 \\
.2131\end{array}$ & $\begin{array}{l}2.65 \\
1.49 \\
1.18\end{array}$ \\
\hline $\begin{array}{l}A B C \\
A B D \\
A B E \\
A C D \\
A C E \\
A D E \\
B C D \\
B C E \\
B D E \\
C D E\end{array}$ & $\begin{array}{r}2.6404 \\
.0000 \\
.1404 \\
7.5623 \\
.0154 \\
.9998 \\
.0623 \\
.1404 \\
.2498 \\
1.5623\end{array}$ & $\begin{array}{r}.6853 \\
.9567 \\
1.2032 \\
.3750 \\
.4353 \\
.3125 \\
1.7513 \\
.8639 \\
1.1531 \\
1.1786\end{array}$ & $\begin{array}{l}3.11 \\
8.90 * * \\
1.18\end{array}$ \\
\hline $\begin{array}{l}A B C D \\
A B C E \\
A B D E \\
A C D E \\
\text { BCDE }\end{array}$ & $\begin{array}{r}1.8910 \\
.0629 \\
.3910 \\
.0160 \\
1.2660\end{array}$ & $\begin{array}{r}.6304 \\
1.1964 \\
.3804 \\
.4620 \\
.4518\end{array}$ & 2.23 \\
\hline $\mathrm{ABCDE}$ & .0169 & 1.0281 & \\
\hline
\end{tabular}

Note. Mean squares are given for subject interactions. Pooled error is used for F tests $(1,217 \mathrm{df})$. 
TABLE 16. Experiment V: Comparison of $F$ Ratios for the Different scoring Systems.

Source
First

Choices
Ranked

Choices 1st and 2nd

Choices

\begin{tabular}{lccc}
\hline A (Sequence) & $112.04 * * *$ & $105.44 * * *$ & $73.01 * * *$ \\
B (Exposure) & $17.56 * * *$ & $21.25 * *$ & $13.41 * * *$ \\
C (Interval) & 1.75 & 0.46 & 3.11 \\
D (Stimulus) & $4.39 *$ & 3.36 & $4.71 *$ \\
E (Similarity) & $3.94 *$ & 0.62 & 0.02 \\
ACD & 1.47 & $5.81 *$ & $8.90 * *$ \\
BCDE & $3.94 *$ & 1.86 & 1.49 \\
\hline
\end{tabular}

Note. Pooled error is used for all $\mathrm{F}^{\prime} \mathrm{s}$.

first choices analysis, decreases in the ranked choices analysis, and disappears in the analysis scoring first and second choices equally. But the situation is rather the same as that for Experiment IV: some suggestions of compensating similarity interactions are present, disallowing the straightforward conclusion that similarity reflects discrimination difficulty. (The reasoning has been that if second choices reduce the similarity effect, we may be able to say similarity reflects S's difficulty in matching his percept with the correct alternative-a discrimination problem.) Subtotals for this experiment, for similar and dissimilar alternatives respectively, are first choices: 335 and 371, for first and second choices: 461 and 459. The difference has disappeared. More second choices from similar alternatives are correct than from dissimilar alternatives, and the $\mathrm{z}$ from an independent proportions test is 2.079. The number of correct second choices from dissimilar alternatives is the amount expected $(88, \mathrm{np}=89.67)$, but $\mathrm{z}$ for the number of correct second choices from similar alternatives is 2.96. If we accept the notion that second 
choices bring out information that $\mathrm{S}$ does not or cannot handle with facility (as other second choice and ranked choice experimentation shows), then this result allows the unequivocal conclusion that similarity is a discrimination difficulty factor.

Overall comparison with Expt. IV shows that the similarity effect is much less pronounced in the present experiment. E supposes this is because of $S^{\prime}$ s extensive practice and familiarity with the experimental situation. A decrease in the similarity difference may suggest increased discrimination acuity: as Ss become more practised they are able to utilise finer differences between alternatives in selecting their response. This is suggested by the figures, and in two ways: not only does similarity diminish, but the sequence effect increases. Ss increase their ability to make use of the preparation that Before sequences allow. The following table of proportions appears to create this impression (main entries are based on 120 responses in Experiment IV and on 320 responses in Experiment V).

TABLE 17. Experiments IV and V: Proportions of First Choices Correct.

\begin{tabular}{|c|c|c|c|c|c|c|}
\hline & \multicolumn{3}{|c|}{ Experiment IV } & \multicolumn{3}{|c|}{ Experiment $\mathrm{V}$} \\
\hline & Sim. & Diss. & Total & Sim. & Diss. & Total \\
\hline Before: & .475 & .608 & .542 & .675 & .728 & .702 \\
\hline After : & .333 & .508 & .421 & .372 & .431 & .402 \\
\hline Total : & .404 & $\cdot 558$ & .482 & .524 & .580 & .552 \\
\hline
\end{tabular}

Experiment $V$ proportions show a clear increase in the sequence effect and a clear decrease in the similarity effect, which is consistent for individual proportions except 
the proportion of correct responses selected from dissimilar alternatives in After sequences. Here the Experiment $V$ figure is less than the Experiment IV figure, and we might suppose reduced short exposure and increased delay have caused the inconsistency, more than counteracting the marked practice effect evident in comparing other entries. The Experiment IV figure, though, is based on fewer responses.

CVCs are identified less well than letters. The difference is not marked, but it appears that the adjustment that $\mathrm{E}$ made to CVC thresholds was too severe.

Forgetting. The main effect of interval is not significant. For first and second choices scored equally, the sequence $\mathrm{x}$ interval $x$ stimulus interaction (ACD) shows uneven forgetting for three sequence $\mathrm{x}$ stimulus combinations but a slight improvement with delaying alternatives for the fourth: letters with alternatives after the stimulus. (The interaction does not occur with analysis of first choices only, and increases of second choice accuracy are generally directly proportional to first choice errors.) An interaction ( $B C D$ ) in Experiment IV, found with all scoring systems, was in this respect of the same nature: letter identification actually improves with longer delays (from 0.4 to 1.8 seconds), but CVC identification does not (at least for exposures of .8 of $\mathrm{S}^{\prime}$ s unadjusted CVC threshold). In Experiment $\mathrm{V}$ this particular interaction is not apparent. The exposure $\mathrm{x}$ interval $\mathrm{x}$ similarity $\mathrm{x}$ stimulus interaction ( $B C D E)$, for the analysis of first choices, is significant (see Fig. 14). Interpreting the interaction in terms of forgetting, it seems that delaying alternatives impairs identification of CVCs the most; forgetting of letters mainly occurs when they are shown at short exposures (.7 of S's threshold) and identified from similar laternatives. The nature of this interaction, while not contradicting earlier suggestions, does not clearly endorse interval 


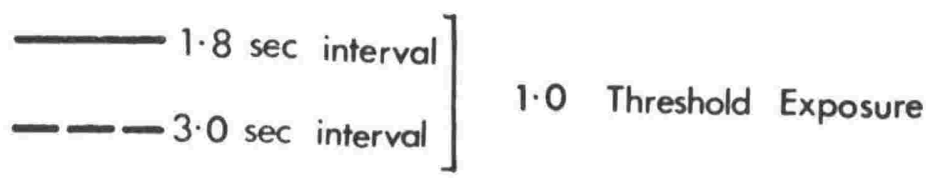

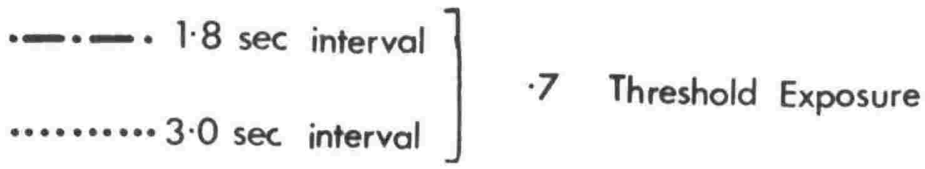

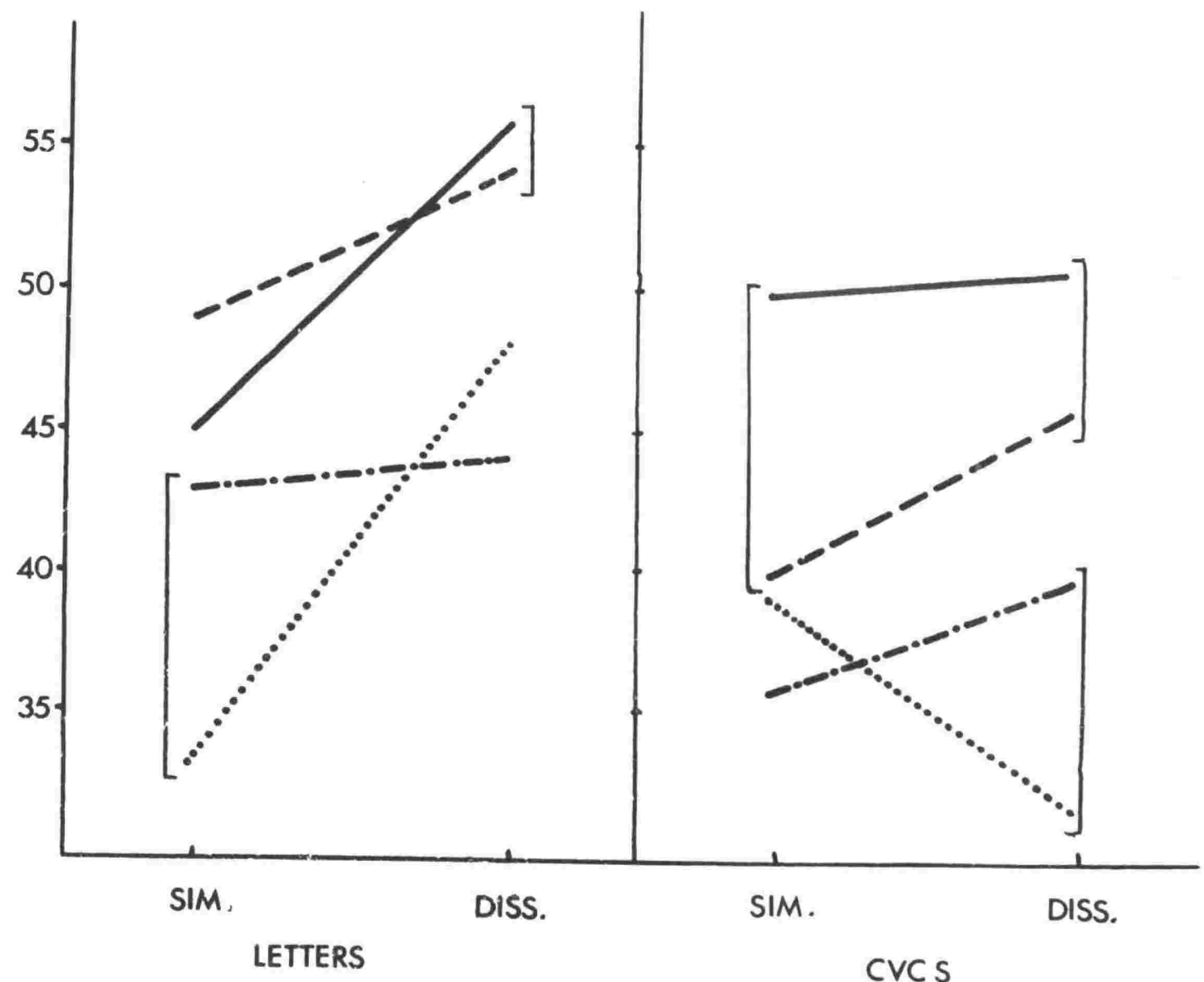

FIGURE 14. Experiment V: Exposure $x$ Interval $x$ Stimulus $\mathrm{x}$ Similarity interaction (First Choices). Bracketed points show forgetting within each exposure $x$ stimulus $x$ similarity condition. 
interaction found elsewhere. We are able to say that forgetting occurs with CVCs rather than with letters (specifically in the Experiment IV BCD interaction and the Experiment V BCDE interaction, and generally with intervalstimulus irregularities for various scoring systems), but a clear interval $x$ stimulus interaction is not to be found.

For first and second choices scored equally, the interval main effect approaches significance $(F=3.11$, F.05 $(1,217)=3.89)$. As the F for first choices only is much lower (1.75), it appears that second choices may be picking up some stimulus information that is rapidly forgotten, although the tendency is slight.

Single and Ranked Choices. In this experiment, 706/1280 (.552) first choices were correct, somewhat higher than correct responses to letters and CVCs in Experiment II (.510), in spite of increased delay of alternatives and shorter stimulus exposures. Practice presumably cancels these factors. The ranks of correct choices were distributed as follows:

$\begin{array}{rllll}\text { Choice: } & 1 \text { st } & 2 \text { nd } & 3 r d & 4 \text { th } \\ \text { No. Correct: } & 706 & 214 & 179 & 181\end{array}$

There are significantly more second choices correct than chance $(.333(1280-706))$, but it seems from the totals that third choices hold no information. The distribution does not tail off.

Stimulus Series. Subjects receive two series of stimuli (letters and CVCs) per session. If the data specific to each session (responses to the same letters and CVCs) are summed over sequence $\mathrm{x}$ exposure $\mathrm{x}$ interval combinations, we can inspect totals specific to each series of stimuli. (The summing is like obtaining treatment totals from the agricultural latin square.) Each total is a balanced sum 
over Ss of experimental conditions so that any differences in totals may show varying difficulty of identification. The major purpose of this procedure is to examine the CVCs prepared from Noble's list of CVCs having frequency association values between $\cdot 10$ and $\cdot 40$. Subjects had seen in an earlier experiment the six series of CVCs chosen from Archer's list (20-30\%); the CVCs chosen from Noble's list were unseen.

TABLE 18. Experiment V: Analysis of Variance, Stimulus Series.

\begin{tabular}{lccl}
\multicolumn{1}{c}{ Source } & df & MS & F \\
\hline Subjects & 7 & 5.5514 & \\
A (Stimulus) & 1 & 5.6407 & 3.81 \\
B (Similarity) & 1 & 5.0625 & $8.22 *$ \\
C (Sessions) & 7 & 1.7299 & \\
AB & 1 & 3.0625 & $6.24 *$ \\
AC & 7 & .9805 & 1.04 \\
BC & 7 & 1.4375 & 1.39 \\
ABC & 7 & 1.9196 &
\end{tabular}

Errors:

\begin{tabular}{|c|c|c|}
\hline A X S S & 7 & 1.4799 \\
\hline B $x$ Ss & 7 & .6160 \\
\hline $\mathrm{C} \times \mathrm{Ss}$ & 49 & 5.2860 \\
\hline$A B \times S S$ & 7 & .4911 \\
\hline$A C \times S s$ & 49 & .8702 \\
\hline $\mathrm{BC} \times \mathrm{Ss}$ & 49 & 1.0319 \\
\hline$A B C \times S s$ & 49 & 1.1849 \\
\hline Total & 255 & \\
\hline
\end{tabular}

The analysis summarised in Table 18. Stimulus and similarity mean squares are the same as before. The mean square for sessions does not seem large (the sessions $x$ subjects interaction, the error term for testing sessions, 
includes most of the variance for sequence, exposure and interval effects). A stimulus $x$ sessions interaction should display any irregularity in accuracy scores for the two series of CVCs chosen from Noble's list, if any irregularity is present, but it would seem this is not the case: $F=1.04$. Examining CVC totals, however, shows that the two CVC series prepared from Noble's list do have lower accuracy: 35 and 36, the mean of other CVC totals (from Archer's Iist) is 43.83. A test of this difference would appear to be in order, as it is the major purpose of the analysis. Taking components of CVC subset stimulus totals ( 35 and 36 versus the other CVC totals) and testing with the pooled C $\mathrm{x}$ Ss and AC $x$ Ss interactions, $F=4.23(F .05(1,98)=3.94)$. Although the separate errors are heterogeneous and the $F$ probability not much beyond. 05 , the C x Ss interaction contains a very large proportion of variance attributable to specific sources, and E would regard justifiable the conclusion that the two series of CVCs chosen from Noble's list differ significantly from those chosen from Archer's Iist. Later checking of CVCs chosen from Noble's list suggests the lower Archer meaningfulness of these CVCs might be the reason for their less accurate identification: these CVCs have an average Archer meaningfulness of $15.86 \%$, all other CVCs were chosen from the 20 to $30 \%$ Archer range. Ss had not seen these CVCs in earlier experiments, but E tends to regard their lower meaningfulness to be the more relevant factor.

SUMMARY

1. Overall accuracy of first choices is a little better than first choices to letters and CVCs in Experiment IV and better than single choices to letters and CVCs in Experiment II.

2. Similarity is significant but not as pronounced as usual. 
When second choices are scored equally with first choices, the similarity effect entirely disappears. The number of correct second choices from similar alternatives exceeds chance levels.

3. Preparation for the brief stimulus exposure is strongly evident.

4. Accuracy of identification is much higher when stimuli are exposed at subjects' thresholds than when they are exposed at $\cdot 7$ of threshold values.

5. CVCs are not as well identified as letters in this experiment, but this result is largely due to stimulus exposures. Two series of CVCs of lower meaningfulness are less accurately identified than other cVC series.

6. The main effect of interval between stimulus and alternatives is not significant, but there are suggestions that forgetting of CVCs occurs at longer delays of alternatives presentations. 
In previous experiments After sequences were started by $S$ depressing a button. In Experiment VI, After sequences are begun by $E$. Ss receiving After sequences in this experiment see first a blank card for 5 seconds, the intervening blue field with aligning marks for 1.8 seconds, then the stimulus, the blue field again for either 1.8 or 3.0 seconds, and finally the alternatives for 5 seconds. Before conditions are the same except for the prior alternatives in place of the blank card. E introduced the After sequence with the following: "I want to know just how much help subjects get from a stimulated retina. Some experimenters think that mild pre-illumination makes the retina sensitive, and this allows after-images to last a bit longer; which, of course, helps you when you're making a choice between alternatives." Subjects seemed suitably impressed by this.

In other experiments, subjects were allowed to form the impression that in After sequences their control of the stimulus exposure by the remote control plunger was probably beneficial for perception of the stimulus. Some Ss remarked that they would rather have $\mathrm{E}$ start the sequences (always Before sequences in earlier experiments) and in Experiment VI the plunger is not used. S can expect the stimulus 1.8 seconds after the presentation of prior alternatives (in Before sequences) or the blank card (in After sequences). The brass plate used to dispense alternatives cards was replaced with a cardboard sheet of the same material and gauge used to mount stimuli and alternatives, with obscured edges strengthened with resin glue and a handle attached for easy dispensing. In After sequences, $\mathrm{Ss}$ saw this blank card before the stimulus exposure. During the interval after the stimulus, E slowly drew back the blank card to display the alternatives when the tube illuminated them. 
Stimulus types shown in this experiment were shapes and figures (single complex figures, $C$ in Fig. 3) that $S$ had seen about three months earlier in Experiment I or II. Exposures were .7 or 1.0 of $\mathrm{S}^{\prime}$ s stimulus thresholds. Thresholds for shapes and figures are about the same. For the $\mathrm{Ss}$ in Experiment VI, the mean threshold for shapes is $2.03 \mathrm{~ms}$ and for figures $2.31 \mathrm{~ms}$, s's threshold for figures in each case being equal to or higher than his threshold for shapes. Alternatives were presented 1.8 or 3.0 seconds after the stimulus, and subjects select one response only.

Experiment VI is a $2 \times 2 \times 2 \times 2 \times 2$ factorial experiment, 8 ss receiving 16 of the 32 treatment combinations to confound the higher order interaction with subject group differences. Four measurements were taken per session, and in each session $\mathrm{S}$ s saw shapes and figures exposed at .7 and 1.0 of threshold values and selected their responses from both similar and dissimilar alternatives. Different types of alternatives are not alternated per trial as in all earlier experiments, but 5 of one type are seen consecutively. Halfway through each stimulus series both similarity and exposure change. One practice stimulus is shown at the beginning of each stimulus series and another immediately after similarity and exposure have changed (all changes are unknown to $\mathrm{S}$ ). Grouping the same type of alternatives together was necessitated by the design: each session was at one level of interval ( $\mathrm{S}$ is then not aware of any change); each stimulus series was with one sequence condition; to maintain each stimulus series in a treatment combination required by the design, similarity of alternatives must vary with exposure, and altering exposure on each trial $E$ thought would be too demanding on administration efficiency. The sessions appeared "usual" to subjects. Sessions of two stimulus series take 11,12 minutes, and one datum is again a sum of 5 responses. 
Subjects were also subdivided, within each group of treatment combinations, into repetitions of administrations: 4 Ss received sessions with only one sequence condition, 4 Ss received sessions with both sequence conditions. A difference in repetitions would suggest a sequence effect builds up slowly; if repetitions are equal, any "adjustment" to the different sequences that $S$ may need is probably complete after the test and practice stimuli have been seen. (The difference is labelled "repetitions" since treatment combinations remain unaltered, and different repetitions refers to different compositions of sessions.)

All Ss see all stimuli twice: conditions of Experiment $\mathrm{V}$ apply here.

RESULTS

Sequence, exposure and similarity are significant main effects in this experiment: identification of the stimulus is better when Ss are prepared, when exposure is long and when selection is easy. The sequence $\mathrm{x}$ similarity interaction (Fig. 15) shows that only similar alternatives before the stimulus facilitate identification; when selection is easy, preparation is not important. Accuracy for shapes and figures is identical.

Interval. The main effect of interval is not significant, although delaying alternatives for shapes slightly improves their identification (interval $x$ stimulus interaction, p .10). The exposure $x$ interval interaction (Fig. 16) shows that delaying alternatives impairs accuracy for short stimulus exposures, but improves accuracy for long stimulus exposures. The effect of exposure is observed only with long delays of alternatives.

In Experiments IV and V, forgetting of CVCs occurred 
TABLE 19. Experiment VI: Analysis of Variance.

\begin{tabular}{|c|c|c|c|}
\hline Source & $\mathrm{df}$ & MS & $\mathrm{F}$ \\
\hline Between Ss & 7 & & \\
\hline $\begin{array}{l}\text { Repetitions } \\
\text { ABCDE (Groups) } \\
\text { Residual } \\
\text { Ss/Groups }\end{array}$ & $\begin{array}{l}1 \\
1 \\
1 \\
4\end{array}$ & $\begin{array}{r}.50 \\
24.50 \\
.13 \\
1.41\end{array}$ & $17 \cdot 42 *$ \\
\hline Within Ss & 120 & & \\
\hline 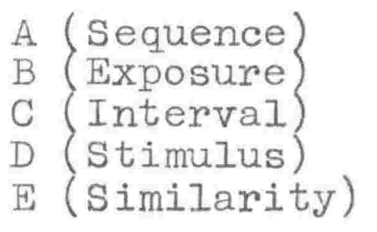 & $\begin{array}{l}1 \\
1 \\
1 \\
1 \\
1\end{array}$ & $\begin{array}{r}12.50 \\
7.03 \\
1.13 \\
.00 \\
22.78\end{array}$ & $\begin{array}{l}13.51 * * * \\
7.60 * * \\
1.22 \\
24.63 * * *\end{array}$ \\
\hline $\begin{array}{l}\mathrm{AB} \\
\mathrm{AC} \\
\mathrm{AD} \\
\mathrm{AE} \\
\mathrm{BC} \\
\mathrm{BD} \\
\mathrm{BE} \\
\mathrm{CD} \\
\mathrm{CE} \\
\mathrm{DE}\end{array}$ & $\begin{array}{l}1 \\
1 \\
1 \\
1 \\
1 \\
1 \\
1 \\
1 \\
1 \\
1\end{array}$ & $\begin{array}{r}.28 \\
.00 \\
.00 \\
13.78 \\
5.28 \\
.03 \\
1.13 \\
3.13 \\
.28 \\
.28\end{array}$ & $\begin{array}{l}14 \cdot 90 * * * \\
5.71 * \\
1.22 \\
3.38\end{array}$ \\
\hline $\begin{array}{l}A B C \\
A B D \\
A B E \\
A C D \\
A C E \\
A D E \\
B C D \\
B C E \\
B D E \\
C D E\end{array}$ & $\begin{array}{l}1 \\
1 \\
1 \\
1 \\
1 \\
1 \\
1 \\
1 \\
1 \\
1\end{array}$ & $\begin{array}{r}.28 \\
.03 \\
.50 \\
.50 \\
.03 \\
.03 \\
1.53 \\
1.13 \\
2.00 \\
.03\end{array}$ & $\begin{array}{l}1.66 \\
1.22 \\
2.16\end{array}$ \\
\hline $\begin{array}{l}\mathrm{ABCD} \\
\mathrm{ABCE} \\
\mathrm{ABDE} \\
\mathrm{ACDE} \\
\mathrm{BCDE}\end{array}$ & $\begin{array}{l}1 \\
1 \\
1 \\
1 \\
1\end{array}$ & $\begin{array}{l}2.53 \\
3.13 \\
1.13 \\
2.53 \\
.50\end{array}$ & $\begin{array}{l}2 \cdot 74 \\
3 \cdot 38 \\
1.22 \\
2 \cdot 74\end{array}$ \\
\hline Residual (w) & 90 & .9250 & \\
\hline Total & 127 & & \\
\hline
\end{tabular}




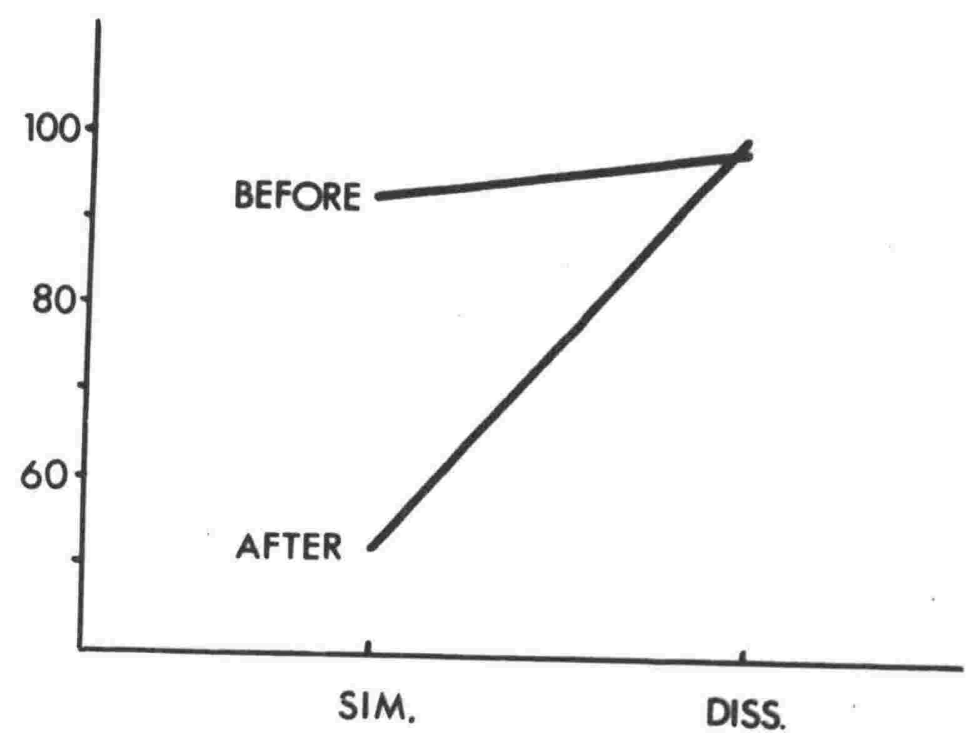

FIGURE 15. Experiment VI: Sequence $\mathrm{x}$ Similarity interaction.

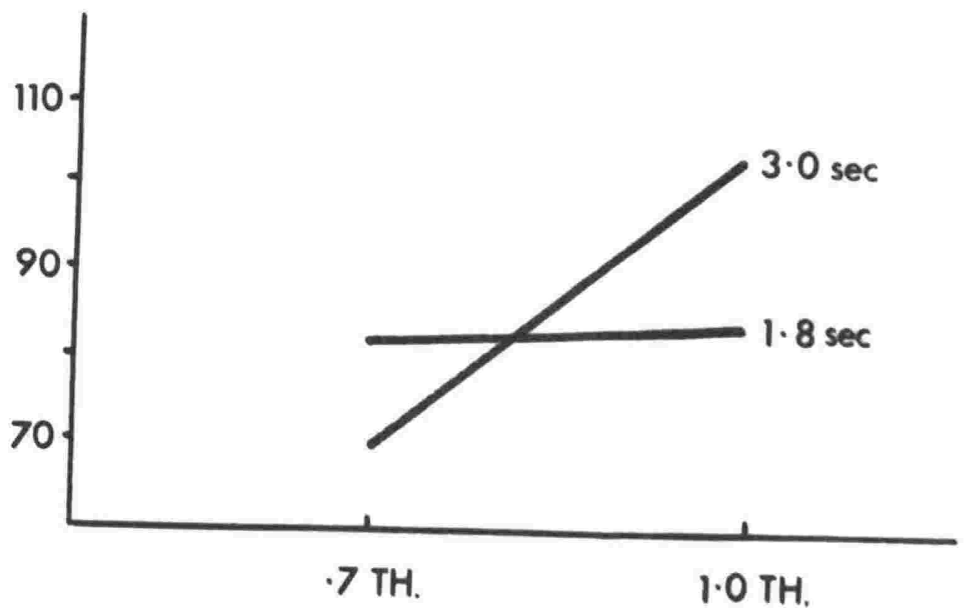

FIGURE 16. Experiment VI: Exposure $x$ Interval interaction. 
at long exposures. The present result with geometric stimuli resembles the earlier effect with letters in Experiment IV ( $0.4-1.8$ seconds delay). In Experiment III (After sequences only), accuracy was a little better at 1.8 seconds delay that at 0.4 seconds delay. That a delay is beneficial at all would seem to suggest that identification is a process which under certain conditions should not be speeded. Apart from CVCs, the overall effect of delaying alternatives for up to 3 seconds is to increase the possibility of correct identification.

The ABCDE interaction $E$ attributes to significant subject differences. Assignment of $\mathrm{Ss}$ was random, and thresholds are, if anything, higher (shapes only) for the lower scoring group. The lower level of accuracy for this group is general, although they contribute more to the sequence $x$ similarity interaction and less to the threshold main effect than the higher scoring group when analyses for two quarter replications are carried out.

Repetitions. Whether Ss receive the same or different sequences per session makes little difference. In Experiment II, Ss in Before sequence conditions showed a small practice effect over halves of stimulus series, but practice, or accomodation, in Experiment VI, if present, is not shown by variance extracted as Repetitions. Series of stimuli are shorter than in Experiment II, and here Ss are more practised before sessions are begun.

Pre-stimulus field. The difference between Before and After sequences in this experiment is the nature of the pre-stimulus field. Before sequences as usual showed S 4 alternatives, After sequences showed $S$ a blank field. E ran 8 sessions with a patterned pre-stimulus field to check 
on the possibility that After sequences may dazzle $\mathrm{S}$ ( the blank card is brighter, and with it $S^{\prime}$ s eye movements much less, than alternatives cards). Multiple figures and pictures were shown at .9 of S's thresholds for these stimuli, with alternatives 3. oseconds after the stimulus exposure. For the pre-stimulus field $E$ inked four rectangles on the blank card, each with diagonals and central spot to encourage eye movements and to equate the brightness of this card with the average alternatives card. Sequence was counterbalanced in sessions for groups of $\mathrm{Ss}$, two stimulus types were identified from either type of alternatives in each sequence condition, confounding the higher order interaction with groups, but E ran no familiarising series and the results show a strong practice effect. Sequence totals (Before and After respectively) are: Before given first: 12,19; Before given second: 33,13. If a three-factor analysis is carried through, sequence, stimulus $\mathrm{x}$ similarity and the confounded interaction are significant. Subtotals indicate the similarity and sequence effect are undoubtedly present, but the practice effect due to absence of the familiarising series prevent exact statements. It can be taken that any dazzle the blank card caused $S$ is at most a relatively minor associated condition of sequence differences in Experiment VI.

\section{SUMMARY}

1. Preparation for the briefly exposed stimulus is once more strongly evident, but only when selection of the stimulus (shapes or complex figures) is made from similar alternatives.

2. Dissimilar alternatives allow easier choices.

3. Stimuli shown at .7 of $\mathrm{S}^{\prime}$ 's threshold are not as well identified as when shown at this threshold, although the difference occurs only when presentation of alternatives is delayed 3 seconds after the stimulus presentation. 
4. Delaying alternatives slightly improves the number of correct identifications, and reviewing results from earlier experiments shows that this improvement applies to shapes, complex figures and letters, but not to CVCs.

5. A subsidiary test indicated the sequence effect in this experiment is not attributable to retinal phenomena. 
In all experiments, stimuli are exposed at values related to S's specific stimulus threshold, and responses, selected from similar and dissimilar alternatives, are location choices in answer booklets. When alternatives are shown before the stimulus, the interval between these presentations is always 1.8 seconds. In Expt. I, Before sequences show $\mathrm{S}$ alternatives before the stimulus only; in other experiments Before sequences show $S$ the alternatives again after the stimulus exposure, making the sequence effect in all later experiments the result of preparation because of the additional prior presentation of alternatives. For Expts. I to IV the lower exposure level is .8 of $\mathrm{S}$ 's stimulus threshold; for Expts. V and VI the lower level is .7. For Expts. III and IV the intervals or delays of alternatives after the stimulus are 0.4 and 1.8 seconds; for Expts. V and VI these delays are 1.8 and 3.0 seconds.

The following table summarises results for the six experiments. The first half of the table indicates the types of stimulus used in each experiment, and the second half presents probabilities $(<.10,<.05,<.01,<.001$ ) for all main effects and for interactions which were significant in at least one experiment. A blank cell indicates the effect is not significant and $p>10$. Entries are not relevant in cells with dashes: interval effects for Expts. I and II (only delays of 1.8 seconds), sequence effects for Expt. III (only After sequence), and in the first half of the table, various stimulus types for Expts. II to VI. Results from multiple choice experiments (IV and V) are given for first choices only, which will be comparable to results from single response experiments. Effects for which p<.10 in only one experiment cannot be traced from the table. 
TABLE 20. Summary of Major Results.

Experiment

\begin{tabular}{|c|c|c|c|c|c|c|}
\hline Stimulus & I & II & III & IV & $\mathrm{V}$ & VI \\
\hline A. Shapes & A & A & $\mathrm{A}$ & - & - & A \\
\hline B. Letters & B & B & B & B & B & - \\
\hline C. Figures 1 & $\mathrm{C}$ & $\mathrm{C}$ & $\mathrm{C}$ & - & - & $\mathrm{C}$ \\
\hline D. CVCs & $\mathrm{D}$ & $\mathrm{D}$ & D & $\mathrm{D}$ & D & - \\
\hline E. Figures 2 & $\mathrm{E}$ & - & - & - & - & - \\
\hline F. Pictures & $F$ & - & - & - & - & - \\
\hline Source & I & II & III & IV & $\mathrm{V}$ & VI \\
\hline Sequence & & $* * *$ & - & $*$ & $* * *$ & $* * *$ \\
\hline Exposure & $*$ & & & & $* * *$ & $* * *$ \\
\hline Interval & - & - & & & & \\
\hline Stimulus & $* * *$ & & $<.10$ & $*$ & $*$ & \\
\hline Similarity & $* * *$ & $* * *$ & $* * *$ & $* *$ & $*$ & $* * *$ \\
\hline Seq. $x \operatorname{Exp}$ & $*$ & & - & & & \\
\hline Seq. $x$ stim. & & $*$ & - & & & \\
\hline Seq. $x$ Sim. & $<.10$ & & - & & & $* * *$ \\
\hline $\operatorname{Exp} . \mathrm{x}$ Int. & - & - & & & & $*$ \\
\hline $\operatorname{Exp} \cdot \mathrm{x} \operatorname{Sim}$ & $* *$ & & & * & & \\
\hline Ex I x Stim. & - & - & & ** & & \\
\hline $\mathrm{E} x I x$ St $x \mathrm{Sy}$ & - & - & & & * & \\
\hline
\end{tabular}


1. Sequence. Preparation for the briefly exposed stimulus is a significant effect in all experiments where subjects identify the stimulus directly from response alternatives. Requiring subjects to remember alternative locations (Expt. I) obscures the sequence effect. Preparation can be said to facilitate difficult discrimination (Expt. VI), and have less (but still significant) effect when the stimulus class is well known (letters, Expt. II). The evidence for set as reduction in range of expectation is indisputable.

2. Similarity. Selecting the exposed stimulus from similar alternatives is a more difficult task than selecting from dissimilar alternatives in every experiment conducted. Analysis of errors shows that this is not a statistical sideeffect of a two-stage discrimination process, and analysis of subjects' residual information (second choices) confirms that discrimination difficulty is tho basis to the similarity effect. In addition, discrimination from similar alternatives is aided by preparation (Expt. VI) and by increasing stimulus exposure a few tenths of a millisecond (Expts. I and IV).

3. Exposure. Identification of stimuli shown at .8 of subjects' thresholds is generally not very much worse than identification at full threshold values, although accuracy at . 8 thresholds is always less (significantly so in Expt. I). When exposures are .7 and 1.0 of subjects' thresholds, the exposure effect is marked (Expts. V and VI).

4. Forgetting. Alternatives were delayed after the stimulus exposure for intervals of $0.4,1.8$ and 3.0 seconds, but there were no significant main effects in four experiments. The three significant interval interactions always involved exposure, but their consistency is not marked. Presenting alternatives 0.4 seconds after the stimulus was generally detrimental to accuracy except for CVCs, and after a delay of 
3.0 seconds, identification was again impaired. The exposure $\mathrm{x}$ interval interaction in Expt. VI suggests forgetting only at short exposures (shapes and figures).

5. Stimulus. All stimulus main effects can be traced directly to subjects' stimulus thresholds. In Expt. I a highly significant stimulus effect disappeared with adjustment for thresholds. In Expt. IV, CVCs were more accurately identified than letters, but in Expt. $V$ when CVCs were shown at shorter exposures than letters, the effect was reversed. In Expt. II preparation facilitated accuracy of CVCs most and letters least. 


\section{DISCUSSION}

We have clear evidence for a beneficial effect of preparing subjects for a briefly exposed stimulus. Preparation or set, experimentally defined as reduced range of expectation, considerably facilitates identification. In the introduction to this study, it was noted that the explanation or locus of set has on occasion been assigned to at least five different sources: facilitation of information handling processes, receptor adjustment, sensitisation of perception, memory processes and response processes. Extra but not always independent explanations refer to stimulus uncertainty, response uncertainty, familiarity with alternatives, stimulus discriminability, response preparation (decision processes), buffer storage decay, selective attention, and perceptual and memory spans.

At the outset, we can exclude those explanations involving terminal response processes (the same for all subjects), gross receptor adjustment (all subjects look through the eyepiece at the fixation point), and forgetting due to sequential reporting of several stimulus dimensions (we have used an identification task). Response preparation cannot be excluded, nor can certain types of forgetting. A type of receptor adjustment could have been implicated in Expts. I - V when subjects in After sequences triggered the stimulus themselves, but not in Expt. VI, and since Expts. II, IV, $V$ and VI show the effect of preparation, as well as the subsidiary test reported in Expt. VI, receptor adjustment can be excluded. (Slow recovery of retinal sensitivity, if important, would act against a preparation effect, since in Expts. I - V subjects in Before sequences get extra stimulation before the stimulus exposure.) We can also exclude here response uncertainty: all subjects, in all experiments, select their responses from four alternatives. 
There remains a variety of explanations to sort out. The effect of preparation has been assigned to any of various stages in the perception-response sequence.

In the Wurzburg view, task-setting firmly structures "mental activity", and where the task instructions are in line with report requirements the accuracy of report is increased. Chapman (1932) pointed out that instructions can influence both the phenomenal perceptual field and surrogative processes. Instructions given after the stimulus can only work on surrogative processes, but instructions given before the stimulus can influence perception as well. With the marked benefit of prior instructions it would seem possible to conclude that perception is facilitated rather than surrogative processes, but some introspections suggested to Chapman that this need not always be the case, and he assigned the influence of instructions to both phenomenal perception and processes intervening between perception and report.

Lawrence \& Coles' experiment (1954) was a direct test of Chapman's hypotheses. They found that identification was better if subjects chose from four alternative verbal descriptions of briefly exposed photographs, but that it made no difference to response accuracy whether subjects saw the descriptions either before or after the exposure of the photographic stimuli. They concluded that it was memory and response processes that were facilitated by alternatives: Chapman's "surrogative processes", and not the traditional sensitisation (which Chapman did allow). It is to be doubted if their experiment shows even this, since identification with alternatives is a selection from four possibilities, while identification without alternatives is a selection from a far greater number of possibilities, and the information that subjects transmitted in responses may have been much the same for both conditions. Where 
this can be calculated (Pollack, 1959; Long, Henneman \& Reid, 1960; Garner, 1962) no such effect in restricting alternatives is observed. Lawrence \& Coles' conclusion about response processes does have some meaning: "the influence of alternatives is primarily on the response variables in the sense of making available or facilitating the occurrence of responses that otherwise would not be made" ( $\mathrm{p}$.213). But to test this, criticisms based on probability arguments should be avoided.

Lawrence \& Coles' other conclusion that the effect of alternatives is on memory rather than perceptual processes can also be questioned. The conclusion is based on the finding that accuracy of response is the same whether alternatives are seen before or after the stimulus, but for response-checking a number corresponding to an alternative verbal description-subjects in Before sequences had to remember numbers of alternative descriptions ( .210$)$, and since we have strong evidence that forgetting of such response requirements easily occurs (Expt. I), Lawrence \& Coles' experiment cannot be considered a test of the sensitisation hypothesis.

Postman does not doubt the effect of instructions on perceptual report, but finds that sorting out the three hypotheses examined by Lawrence \& Coles (perceptual discrimination per se, immediate memory and response) "an important unresolved question" (1963, p.50). Lawrence, writing in the same volume, developed a coding hypothesis: the "effective" stimulus for overt behaviour is a single coded stimulus, but when a complex stimulus is shown (as in Kulpe, Chapman, Lawrence \& LaBerge) and report required on several attributes, separate and successive codings are necessary for each overt response. Instructing a subject to report on one attribute "ensures not only the elicitation of that coding response but also that it will occur first" 
(1963, p.199), and whether the instructions are given before or after the stimulus does not matter: "In both cases the proximal stimulus and its trace must be available before the coding responses can operate" ( $p .199)$.

Common to Postman's and Lawrence's expositions is immediate memory. The stimulus information can be organised if "available". Anderson (1960) and Sperling (1960) examined this contention with partial span methods, and found that where only part of a multiple stimulus was required for report, percent correct responses decreased as post-stimulus instructions were progressively delayed. Sperling exposed up to 12 letters, arranged in rows, for $50 \mathrm{~ms}$ and asked subjects to report one of the rows according to the pitch of an instruction tone (high tone for top row, etc.) and found accuracy of report fell off as instructions were delayed up to 1 secord after the stimulus exposure.

If the stimulus exceeds memory capacity, the subject must select a part to remember (Sperling, 1960, p.23) or to rehearse (Anderson, 1960, p.220) but before the sensory aspect of the stimulus has declined (the image still articulated), this selective memorising would not yet be necessary, and Lawrence's pre-coding conditions would be relevant. For Sperling and Lawrence, instructions act on the proximal stimulus, and a difference between pre- and post-stimulus instructions will occur only when immediate memory cannot store the stimulus completely enough for a fully accurate report. Although these and other authors stress the coding or selective memorising effect of instructions, it may be noted that they have manoeuvred themselves back into a position where instructions influence perception. If immediate memory refers to the proximal stimulus, it is difficult to discover how perception and immediate memory can be separated, and in fact Broadbent (1957) prefers not to do so. Memory, if one adopts concept 
parsimony, can be effectively distinguished from perception only after some coding or transform has operated on peripheral stimulus representation, and even then a distinction between perception and immediate memory depends on definitions.

Returning to the facts, we can note that instructions before a stimulus yield greater accuracy of report than instructions either after, with or very slightly before a stimulus exposure (Lawrence \& LaBerge, 1956, Expt. I and Expt. I vs. II; Sperling, 1959, Tables 7, 8 and 9; 1960, Figs. 3, 5, 6, 7 and 8; Brown, 1960, Conditions I and II; Haber, 1964, p.403). In sperling's data, the fall off in accuracy as instructions are delayed shows both that the sensitisation process is not immediate, and that if instructions are presented after the stimulus but before image articulation degenerates, appreciable amounts of information may be recorded, although not as much as when instructions are given in time for sensitisation to be fully effective (as he realised, p.24).

The present experiments use identification of a single stimulus to show a very noticeable effect of preparation. Although attention and memory spans are involved in sensitisation experiments where a complex stimulus is shown (Rubin, 1913, p.386; Titchener, 1915, p.260), span for a single stimulus, foveally perceived, is hardly relevant. But we do have evidence that delay of response alternatives affects perceptual report. The delay has several effects; forgetting (decrease in accuracy with increasing delay) is only one of these.

If response alternatives are given visually, and are seen through the tachistoscope eyepiece, conditions are obtained where masking of the stimulus by the following alternatives presentation is possible. Experiments on contour masking (Werner, 1935; Averbach \& Coriell, 1959; 
Schiller \& Wiener, 1963; etc.) superimpose one contour on another to inhibit one of the contours, and do not closely resemble the present conditions (although subjects' eye movements may simulate this effect). A white clear field phenomenally masks a pattern previously exposed if the interval is less than $65 \mathrm{~ms}$ and is dark (Humphrey, Dawe \& Mandell, 1955), and many types of following fields tend to mask a stimulus if the interval is white ( 1.6 foot-candles) and $40 \mathrm{~ms}$ (Schiller \& Wiener, 1963). The tachistoscopic conditions most related to the present ones appear to be those of Eriksen \& Hoffman (1963, Field I conditions), who found that recognition of a letter was impaired when a bright field followed the letter after a dark interval of up to $5 \mathrm{~ms}$, with or without an adapting field (Fig. 1, p.491, Field I, conditions A, B, C), but that when there was a continuous adapting field (condition D), no improvement occurred with increasing interval. There was, however, a slight overall tendency for recognition to improve up to delays of $450 \mathrm{~ms}$, and this figure is of direct relevance to the present experiments.

Masking for the condition of Eriksen \& Hoffman most similar to the present ones was nonexistent-the second field was even slightly facilitative. If we can extrapolate to the present conditions (different fixation point, different tubes, more complex informational stimuli, alternatives rather than blank field, etc.) we can tentatively exclude masking as a factor in the detrimental effect that quickly presented alternatives have on accuracy of identification.

In our experiments, the interval main effect was never significant, although there was a suggestion of an interval main effect in Expt. V (first and second choices, p<.10, intervals of 1.8 and 3.0 seconds). There were three significant interval interactions, and they always involved 
exposure. If the subtotals for the different intervals are examined for separate exposure levels, it is observed that for short exposures of stimuli (.7 and .8 of subjects' thresholds) a delay of alternatives of 1.8 seconds is better than either a delay of 0.4 or 3.0 seconds, but for stimuli exposed at subjects' thresholds, the delay effect is quite specific to the type of stimulus seen: CVCs are forgotten over longer delays ( $1.8 \mathrm{vs} .0 .4$, and $3.0 \mathrm{vs} .1 .8$ seconds), but delay facilitates identification of letters, shapes and complex figures. CVCs appear to be a different type of stimulus; they are not so unitary and their identifcation is facilitated more by preparation. In Expt. V (first and second choices), where there is a suggestion for an interval main effect, the sequence $\mathrm{x}$ interval $\mathrm{x}$ stimulus interaction is significant (it is not for first choices only), and this may show that preparation for CVCs prevents forgetting of these stimuli and that the reverse holds for letters; but the interrelationships of correct second choices do not show this trend (if anything, quite the opposite), and it is felt that we cannot say anything definite about this interaction.

Perhaps it is true to say that CVCs are forgotten, but for other stimulus types delaying alternatives seems to assist identification: for long (threshold) exposures delays of up to 3.0 seconds assist, but for short (threshold proportion) exposures delays of 1.8 seconds assist, and longer delays allow forgetting of all stimulus types. Making the obvious generalisation, we might say that less well perceived stimuli do not remain available for decision as long as better perceived stimuli. Stimulus consolidation and/or decision time appear to be more important factors than forgetting.

Allowing time for successive decisions is important in reaction time studies, but in the present stimulus 
identification experiments only one stimulus is shown and only one response required (in Expts. IV and V, where ranked responses are required, the effect of delaying alternatives is the same). Longer time for decision improves identification of shapes, letters and figures, irrespective of preparation. Pollack (1959) noted a decrement in accuracy when alternatives were given 1 second after the stimulus (auditory spondee) had been presented in a perceptually difficult signal/noise ratio $(-17 \mathrm{db})$ but not in an easier $\mathrm{S} / \mathrm{N}$ ratio $(-15 \mathrm{db})$ or for longer delays of alternatives, under which accuracy fell off gradually over 16 seconds. The anomolous decrement in his data seems to resemble the present results, and he quotes his subjects as saying "We couldn't get a good enough picture of what we heard before the two response alternatives were presented" (1959, p.1505). If we take this comment at face value, stimulus consolidation appears to be involved.

We may conclude, then, that the evidence for preparation in these stimulus identification experiments is not at all vitiated by forgetting. What suggestions we have for interval effects are of quite minor relevance, and can in no way explain the extremely pronounced effects of preparation. Any sequence $\mathrm{x}$ interval interactions that Lawrence and Sperling would predict do not appear. A suggestion in favour of a forgetting hypothesis of set is incipient in the identification of CVCs, but only incipient, and sequence $x$ stimulus interactions cannot challenge the above generalisation that in these experiments preparation is far more pronounced than forgetting. When delaying alternatives actually improves identification, sometimes even for CVCs, we can hardly allow the notion that preparation merely prevents forgetting. 
The preparation effect is still open to several explanations. Of the variety listed at the beginning of this discussion, these explanations can be considered possible: facilitation of information handling processes, sensitisation of perception, stimulus uncertainty (the present experimental definition of set), familiarity with alternatives, stimulus discriminability and response preparation (or decision processes). As explanations of preparation for single stimulus identification, some of the contenders are perhaps more likely than others. It is thought that several possibilities have been excluded: response uncertainty, memory decay, perceptual and memory spans and receptor adjustment. (Selective attention, while an important determinant of perception, and certainly an explanation of set in many situations (see Garner, 1962, p. 132-133), can only mean sensitisation in the present context where subjects can expect the stimulus in central vision and with full attention.)

Set has been manipulated by reducing the range of expected stimuli. Prepared subjects can expect one of four specific alternative stimuli; unprepared subjects can expect any stimulus of a certain type (shapes, letters, etc.). Since responses are always made from four alternatives, the increase in accuracy of identification is directly related to reduction of stimulus uncertainty or stimulus expectation.

NUNERICAI STIMULUS UNCERTAINTY

It is conceivable that the improvement in identification as stimulus uncertainty is reduced might be a similar phenomenon to improvement in identification as the number of allowable responses is reduced. Reduction in response uncertainty, where stimulus uncertainty is held constant, is largely though not entirely a probability matter. Where stimulus and response uncertainty vary together, the 
organism tends to transmit constant information (Garner, 1962), and Miller placed Kulpe's and Chapman's results alongside results from absolute judgment studies with the conclusion that "accuracy of judgment on each attribute decreased as more dimensions were added" (1956, p.89). The constant information transmission observed in many situations is perhaps a generality of high validity, but there are likely to be severe restrictions in situations where external and subject information constructs do not mesh. In Long, Henneman \& Reid's (1960) Experiment I, where stimulus and response uncertainty varied together, information transmission calculated from external probabilities was not constant when $\mathbb{N}$ varied, but was highest when subjects were faced with most alternatives. More intricate analyses may perhaps show greater uniformity of information transmission, but as a working hypothesis it is easy to accept the view that subjects "hold" an optimal amount of stimulus possibility in readiness for perception of a single stimulus. In Pollack's data (1959) there is only a slight stimulus uncertainty effect (it was not tested) and as Pollack comments, it is difficult to allow less stimulus uncertainty than response uncertainty to test the relative effectiveness of each-subjects have to be deliberately misled. In all our experiments, response uncertainty is theoretically constant, and it is to stimulus uncertainty that we assign the effect of preparation.

Suggestions that identification accuracy is at least partly a numerical function of stimulus uncertainty is found in the sequence $\mathrm{x}$ stimulus interaction of Expt. II: preparation for letters is not as marked as it is for other stimulus types, and since the stimulus population for letters is the smallest, the numerical uncertainty theory is not contradicted. A similar tendency occurs in Expt. IV (preparation is a little less obvious for letters than for CVCs) but it does not occur in Expt. V when subjects are more 
practised. Perhaps uncertainty radically changes as familiarity with the stimulus population increases, whether the actual populations are large or small. For less senior Psychology students, initial familiarity with CVCs is bound to be less than familiarity with letters. If it is accepted that complex figures can vary in more ways than shapes (the figures are "open" shapes that possess internal differentiation), we might postulate a larger population of possible and discriminable figures than of shapes, and yet the overall identification and preparation effect for these two stimulus types are very similar and even identical in Expt. VI.

Long, Henneman \& Reid (1960, Experiment II) say a preparation effect occurred mainly when subjects identified the degraded letter from two alternatives rather than from four or eight, although this effect was not strong enough to produce a preparation $x$ alternatives interaction. At first sight, this appears to bolster the numerical uncertainty theory, but it occurs when the response population is 26 rather than 14, and then only because identification when unprepared is zero, and this invalidates their conclusion.

We have insufficient data to firmly reject the numerical uncertainty view. We can note the absence of distinctive results in favour of this view, and also note that it makes no reference to qualitiative or discriminative uncertainty.

However, reduction of stimulus uncertainty is the present experimental definition of set, or at least the reduction of expectation from an indefinable number of stimulus possibilities to a fixed number of specific possibilities. It has been thought that subjects, knowing the stimulus will be one of four alternatives can, when prepared, make use of specific characteristics of the alternatives to improve identification scores. Factorial 
designs were employed in the hope that sequence interactions would allow precise conclusions about the influence of set, but we have only three of these: interactions of sequence with exposure (Expt. I), stimulus (Expt. II) and similarity (Expt. VI). The first, the sequence $x$ exposure interaction in Experiment $I$, occurred in the absence of a sequence main effect, and the interaction is not apparent in other experiments. Also, the interaction appears to be due to uniquely poor identification scores in one of the subgroups, and any theoretical importance of this interaction is disregarded.

\section{STIMULUS DISCRIMINABILITY}

Sequence $\mathrm{x}$ similarity interactions are the interactions that a discrimination approach to set would predict. Preparation may help selection of the stimulus when discrimination is difficult, but be of less help when discrimination is easy.

This is the case in Expt. VI where a preparation effect is observed only for selections made from similar alternatives. The interaction is marked, but does not appear in other experiments, and perhaps the effect depends in a subtle way on overall discrimination difficulty and practice. In Expt. II, the similarity effect is twice as great for Before sequences as it is for After sequences, and preparation for selection from dissimilar alternatives is much greater than preparation for selection from similar alternatives. Expt. IV subtotals show a pattern closer to Expt. VI than to Expt. II, and the Expt. V subtotals do not show any suggestion of a differential preparation effect. If proportions for sequence $\mathrm{x}$ similarity conditions for the various experiments are compared, it is noted that Expt. VI proportions are much the same as proportions in other experiments except for the After sequence, dissimilar 
alternatives condition which is 12\% higher in Expt. VI than in any other experiment. The sequence $\mathrm{x}$ similarity interaction has appeared in this experiment because of an increase in this condition rather than for any other reason. Apart from greater practice, this increase can be associated with the structure of the stimulus series. In every experiment except Expt. VI, subjects identified the stimulus from the same type of alternatives in blocks of five trials. Expectancies about the difficulty of the required

discriminations can explain the interaction, and the lack of this interaction in other experiments, if we allow the phenomenon of the development of sets in the early trials of a task (Harlow, 1949; Eckstrand \& Wickens, 1954; Schoenfeld \& Cumming, 1963) where set refers to the type of expected discriminations. When a block of easy discriminations begins, subjects can be set to observe gross differences and to neglect minor differences that would be important only in difficult discriminations (Krulee, 1958). This type of set would not be relevant in Before sequences, which allow even more specific expectancies, and would not affect discrimination from similar alternatives in After sequences (as Krulee would predict) unless subjects were not responding with maximum accuracy when series were mixed as in other experiments.

The explanation offerred requires that subjects can rapidly develop expectancies, and presupposes (perhaps rightly enough in this case) that "irrelevant" stimulus characteristics can be effectively ignored (Archer, 1954; Green \& Anderson, 1956). Our hypothesis would be that where easy discriminations are grouped in blocks, preparation is of little value. Under these conditions, only when discriminations are difficult does preparation benefit the identification of stimuli. The hypothesis results from the postulation of rapidly developing expectancies, the ability of subjects to disregard intricate stimulus characteristics, 
the relative unimportance of different exposures (exposure changed with similarity between blocks), and as well, the presumption that the perception and retention of intricate but irrelevant stimulus characteristics impairs identification.

However, in other experiments subjects cannot form such expectancies, and we need a more complete explanation than the discrimination hypothesis.

In all experiments that show the effect of preparation, identification when subjects are prepared and select the stimulus from similar alternatives results in accuracy nearly as good and usually much better than when subjects are not prepared and select the same stimulus from dissimilar alternatives. Preparation can make up any deficit in identification accuracy that discrimination difficulty may impose. This quite general conclusion applies to all experiments that tested the preparation effect (Experiments II, IV, V and VI).

The conclusion has a similar operational meaning to Chapman's and Lawrence's conclusions. Lawrence \& Coles reasoned that selection with presentation of alternatives after the stimulus must be on the basis of the memory trace which quickly loses differentiation, and the hypothesis would predict a sequence $x$ similarity interaction (which did not occur in their experiment). In Long, Henneman \& Reid's (1960) third experiment, the preparation effect is greater with the more discriminable (less degraded) letters, although this is rather a different discriminability than the present similarity of alternatives.

It seems plausible to suppose that preparation is less necessary for identification under easy discrimination conditions, but perhaps overall discriminability must be of 
a particular nature and subjects possess a particular level of expectation before this may be observed. Our results generally show that preparation facilitates identification even when discrimination is easy. Where the fading trace and stimulus discriminability hypotheses overlap, there is little evidence for either, and the Expt. VI interaction appears to need at least an expectancy hypothesis to explain the discrimination hypothesis.

THE PREPARATION EFFECT

Despite the foregoing theorising, explanations of the preparation effect involving discriminability, stimulus uncertainty and forgetting cannot be categorically excluded. Versions of these theories are contingent with the experimental definition of preparation, and with suitable modifications, may help to explain the present results.

In the task of identifying the stimulus, prior presentation of alternatives lessens stimulus possibilities, permits subjects to discover discriminable stimulus aspects, and makes possible some sort of immediate decision when the stimulus is presented. The three preceding theories are involved. Within the context of the present task, expressing the discriminability hypothesis in the above manner makes its operational implications the same as those for explanations invoking sensitisation, information handling processes, familiarisation with alternatives and response preparation. The explanations are verbally distinct, certainly; but they make very similar predictions.

1. A complete explanation of the preparation effect cannot be assigned to discriminability unless qualitative stimulus uncertainty, or specification of redundancy, is such that extra specification by preparation is no longer beneficial. 
To gain this extra specification, preparation merely becomes extra-experimental, giving subjects permanent expectancies before trials begin. As we noted earlier, stimulus discriminability cannot explain the preparation effect without involving expectancy.

2. Lawrence and Sperling would expect a preparation effect only because of rapid decay of stimulus information. But we have found this decay of stimulus information to be quite slow, and delaying alternatives has much less effect on identification accuracy than does preparation. Clear sequence $x$ interval interactions do not occur, in opposition to Lawrence's and sperling's theorising, and delaying alternatives, which has diverse effects, suggests that even stimulus consolidation, not only decay, may be involved. It is possible that the forgetting hypothesis can never be tested: if preparation develops slowly, and if critical forgetting occurs more quickly than preparation develops, and if also these two processes are even slightly mutually inhibitive-all of which appear likely-then we cannot test the present forgetting hypothesis. In Lawrence's view (1963), report depends on a coding response to the "proximal stimulus". If the information present during the interval between stimulus and alternatives refers to the "proximal stimulus" and this is not coded until alternatives have been presented, then judging by our results, the stimulus if perceived is nearly always available when alternatives are presented, and the forgetting hypothesis has no experimental foundation.

3. A coding interpretation is implicated in any theorising based on preparation. If we accept Lawrence's plausible view that an overt response is made from a prior coding response (response preparation), then coding may be initiated before stimulus reception by prior presentation of alternatives. 
Both Lawrence and Sperling hold the view that response can be made (coded or prepared) on the availability of stimulus information. Our own explanation (of single stimulus identification) contradicts this view: the coding response, or whatever internal process this involves, is facilitated by preparing the subject, and there appears to be no adequate evidence to suggest anything else. Coding of a complex stimulus after it has been presented does not increase accuracy (Klemmer, 1964), and our own evidence suggests that only when response preparation can begin before stimulus presentation does coding have any beneficial effect. Accuracy is increased only by preparation or expectation.

This conclusion does not fully rule out any of the hypotheses listed earlier $(p \cdot 122)$. Prior presentation of alternatives facilitates information handing processes (initiates coding), gives familiarity with alternatives, allows response preparation to begin, indicates the type of discrimination required, reduces numerical and qualitative stimulus uncertainty, and, if we allow it, sensitises perception. Choice of a generic description of the common element in these explanations is a matter of personal preference, but perhaps preparation implies all of them. 
SUMMARY

A series of experiments examined the effect of preparation on a stimulus identification task. Several types of stimuli were employed (solid shapes, letters, single complex figures, nonsense syllables, multiple figures and pictures of common objects), with three stimulus exposures, three enforced response delay times, two levels of stimulus discriminability and three types of response scoring, in an attempt to specify the influence of preparation or set in perception.

Subjects were required to identify a briefly exposed stimulus by checking a matching location for one of four alternative stimuli. Stimuli were shown in a three-field tachistoscope at exposures equivalent to or less than subjects' limits method thresholds and responses were selected from similar or dissimilar alternatives, either 0.4 , 1.8 or 3.0 seconds after the stimulus had been exposed.

Preparation for the stimulus exposure, by presenting alternatives before as well as after the stimulus, greatly increased accuracy of identification from alternatives in four experiments. When subjects ranked the alternatives in order of probable stimulus matching and correct choices of second ranks included in analyses, difficulty of discrimination between alternatives disappeared, but the preparation effect remained. When the interval between stimulus and alternatives was increased, some forgetting occurred at short exposures, but at longer exposures, delaying alternatives improved identification except for nonsense syllables. When alternatives are given only before or after the stimulus, the preparation effect was lost. Preparation most facilitates the identification of nonsense syllables and least facilitates the identification of letters. 
These results were discussed in conjunction with theories of perceptual set. In particular, two theories were rejected. First, the theory that set merely prevents forgetting, and secondly, the theory that identification is influenced only by response uncertainty. It was not possible to reject other theories, although it was pointed out that some modification is required for the discriminability and numerical stimulus uncertainty theories, perhaps by incorporating hypotheses about expectancy, before either theory can describe the data adequately. A predilection for an internal response facilitation theory was expressed: preparation for a stimulus event allows the perceived stimulus to be readily interpreted; whatever processes are necessary for response can be initiated by preparation before actual stimulus reception. Evidence favouring this view against other views is discussed. 


\section{APPENDIX}

\section{APPENDIX 1}

The stimulus and alternatives are illuminated by mercury-argon cold cathode tubes with white phosphor coating. Peak illumination is reached in less time than $0.5 \mathrm{~ms}$ and decays within $0.3 \mathrm{~ms}$. With a yellow filter, illumination falls to zero within $3.0 \mathrm{~ms}$, but shows a kick-back of about $5 \%$ total illumination that only disappears in 2.5 to $3.0 \mathrm{~ms}$ (this latter decay after the blue tube of the adapting field has reached peak). The shape of onset traces remains unaltered with blue or green filters but with the green filter no decay is registered. Both onset and offset lags are due to phosphorescence, since the blue tube shows almost fully rectangular traces.

Exposures longer than $1.0 \mathrm{~ms}$ add even illumination after a $10 \%$ intitial decay from peak. Successive exposures usually give traces of almost identical amplitude except for $2 \%$ of exposures which show augmentation of initial peak illumination of up to $40 \%$. This is perhaps due to the halfrectified voltage supply. Occasionally small peaks of up to $10 \%$ total illumination were observed between onset and offset, perhaps for the same reason. The augmented peak illumination, and its frequency of occurrence, may explain subjects' spontaneous comments on the ease of perception of some stimuli. The irregularity is unfortunate, since it increases error terms in analyses but, of course, applies to all experimental conditions equally.

The phosphorescence yields a medium whiteness and the off-white cards for alternatives are seen faintly yellowish rather than a dull white. After the 20 minutes required for warm-up, the illumination of alternatives is 3.5 foot-lamberts, range $0.2 \mathrm{ft}-\mathrm{l}$. , at the eyepiece, and the blue adapting field 
$0.55 \mathrm{ft}-1$. , range $0.03 \mathrm{ft}-1$. After long use, there is a slight tendency for brightness of the alternatives illumination to drop, but $0.3 \mathrm{ft}-1$. appeared to be the maximum decrease.

The oscilloscope (Telequipment, D43 Type C) was loaned by the University Physics Department; the rapid photomultiplier by Mr. G.T.Goodger; and the brightness meter (Luckiesh-Taylor) by the Photometry Section, P.E.L., D.S.I.R., Lower Hutt. The author wishes to record his thanks for the loan of testing equipment, to Physics staff, and to Mr. H.S.C. Clarkson for kindly supplying the following description of tachistoscope design.

Rapid decay mercury-argon tubes operating from 1200 V.D.C. are used for stimuli and blanking lights and are each switched via a $6 \mathrm{BL} 7$ valve, each valve having its anodes, cathodes and grids paralleled.

The tachistoscope was designed as a Blank-StimulusBlank conception and used a 5963 valve as a monostable flipflop to trigger a bistable flip-flop using a $12 \mathrm{AU} 7$ valve. The bistable controls Stimulus and Blank; the stimulus when the 5963 is in the astable state, and the blank when the 5963 returns to its stable position.

A modification was then added. The positive going edge of the bistable flip-flop as it returns to the stable state is used to trigger two cascaded $12 \mathrm{AU} 7$ monostable flip-flops. The first flip-flop is used to continue to hold the blank light on for a set period after which time the second $12 \mathrm{AU} 7$ flip-flop operates. This has a relay in its non-stable anode circuit which is de-energised when the flip-flop is triggered. Relay contacts break the grid circuit of the 6BL7 controlling the blank light and applies a negative potential to the grid. Another set of contacts also apply 
Field:

Adapt.

off

off

off

Stim.

on

Alt.

on

Adapt

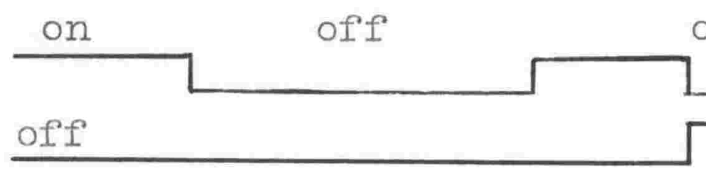

off

off

Stim.

on

Alt.

on

on

A

B

C D

E

F

FIGURE 17. Field conditions schematically, time (not on scale) left to right. Two sequences are shown:

alternatives before the stimulus (top), and alternatives both before and after the stimulus (bottom). (For Expt. I, prepared subjects saw alternatives only before the stimulus.)

Only one field is seen at any one time. Alternatives ( $A B, E F$ ) are shown for 10 (Expt. I) or 5 (Expts. II - VI) seconds. $\mathrm{BC}$, the time between prior alternatives and the stimulus, is always 1.8 seconds. $\mathrm{CD}$, the stimulus exposure, is related to subjects' thresholds. DE, the interval factor, is either $0.4,1.8$ or 3.0 seconds. (In Mr. Clarkson's description, the adapting field is referred to as Blank, and alternatives as Stimulus 2.) 
a negative potential to the bistable circuit to prevent stimulus 1 operating. The positive going edge as this anode becomes nonconducting is applied to the grid of a further $6 B L 7$ to bring on a second stimulus. On reversion to stable state, stimulus 2 6BL7 becomes nonconducting and blank light is restored. This achieves "A" sequence.

For "B" sequence the first $12 A U 7$ flip-flop of the modification is switched out of circuit. What was previously the second $12 \mathrm{AU} 7 \mathrm{flip-flop}$ is triggered and stimulus 2 is illuminated. On return to its stable state a simple timing delay unit is operated from a further set of relay contacts in the now energised anode circuit relay. After the required delay time the original tachistoscope "normal" sequence is triggered by a contact operating a further relay whose contact applies an earth positive 300 volt H.T. momentarily to the start circuit. This second relay is operated via a capacitor hence the momentary operation. The capacitor is later discharged via a resistor so that a small reverse current flows insufficient to operate the relay (this occurs when the relay is de-energised during the next astable period).

A further modification was later added to obtain a "C" sequence or what might be called an " $A$ " plus sequence. A 2-contact push button is used. One contact applies negative 300 volts to the second $12 \mathrm{AU} 7$ in the modification chassis and stimulus 2 operates, with blank automatically suppressed. The second contact of the push button sets a further electronic timer delay circuit in operation. After stimulus 2 has restored to blank, plus a period of blank illumination, a further relay is momentarily operated via a capacitor to give a start signal to the A sequence. To avoid interaction, these last relays and timers are operated from individual 90 volt H.T. batteries. 
APPENDIX 2

The wild scoring subject excluded from Experiment I received the After sequence, Short exposure condition, and was in group II. His total score was $46 / 60$, the highest subject total and 2.50 SD from the mean of all Ss' totals. With this subject, the group variance was 53.0625; with the new subject 2.9167 , the total Ss/Groups variance falling from 78.7804 to 28.5626 , and was then a homogeneous error term. Presumably the excluded $S$ was a "cautious" one, his responses to method of limits presentations being much more delayed than other subjects' responses, with a consequent greater discrepancy between the limits threshold measure and forcedchoice accuracy. His thresholds were, in fact, rather high. Subsequent testing for another experiment (when his accuracy scores were in the usual range) showed the original thresholds were exceptionally inaccurate. For Expt. I, his thresholds for letters and CVCs were 13.0 and $8.5 \mathrm{~ms}$; on retesting for Expt. IV they were 6.0 and $5.0 \mathrm{~ms}$ respectively. (When the difference was pointed out to him, he thought he was not so "wound up" during vacation, when Expt. IV was run, and said he could notice a change in threshold trials for Expt. IV.)

Original s

\begin{tabular}{|c|c|c|c|c|c|c|c|}
\hline \multirow[b]{2}{*}{ Seq. } & \multirow[b]{2}{*}{$\operatorname{Exp}$} & & \\
\hline & & sim. & diss. & mean & sim. & diss. & mean \\
\hline \multirow{3}{*}{ Before } & short & 51 & 46 & 48.5 & 51 & 46 & 48.5 \\
\hline & long & 39 & 59 & 49.0 & 39 & 59 & 49.0 \\
\hline & mean & 45.0 & 52.5 & 48.75 & 45.0 & $52 \cdot 5$ & 48.75 \\
\hline \multirow{3}{*}{ After } & short & 40 & 59 & $49 \cdot 5$ & 29 & 41 & 35.0 \\
\hline & long & 54 & 66 & 60.0 & 54 & 66 & 60.0 \\
\hline & mean & 47.0 & 62.5 & 54.75 & $41 \cdot 5$ & 53.5 & 47.5 \\
\hline
\end{tabular}

Comparing subtotals indicate the change. It would appear that with the original subject the sequence $x$ exposure interaction would change, and that the After sequence total would be higher than the Before sequence total. 
APPENDIX 3

TABLE 21. Experiment II: Analysis of Variance.

\begin{tabular}{|c|c|c|c|}
\hline Source & $d f$ & MS & $\mathrm{F}$ \\
\hline Between Ss & 23 & & \\
\hline $\begin{array}{l}\text { A (Sequence) } \\
\text { B (Exposure) } \\
\text { C (Threshold) } \\
\text { AB } \\
\text { AC } \\
\text { BC } \\
\text { ABC } \\
\text { Ss/Groups }\end{array}$ & $\begin{array}{r}1 \\
1 \\
1 \\
1 \\
1 \\
1 \\
1 \\
16\end{array}$ & $\begin{array}{r}121.9219 \\
13.5469 \\
14.6302 \\
1.8802 \\
.4219 \\
.1302 \\
.0052 \\
5.2708\end{array}$ & $\begin{array}{l}23.13 * * * \\
2.57 \\
2.78\end{array}$ \\
\hline Within Ss & 168 & & \\
\hline $\begin{array}{l}D \text { (Stimulus) } \\
\text { AD } \\
\text { BD } \\
\text { CD } \\
\text { ABD } \\
\text { ACD } \\
\text { BCD } \\
\text { ABCD } \\
D \times \text { Ss/Groups }\end{array}$ & $\begin{array}{l}3 \\
3 \\
3 \\
3 \\
3 \\
3 \\
3 \\
3 \\
48\end{array}$ & $\begin{array}{r}6.0330 \\
12.3941 \\
1.9635 \\
2.2413 \\
2.7691 \\
1.7255 \\
2.9358 \\
3.3961 \\
3.8125\end{array}$ & $\begin{array}{l}1 \cdot 58 \\
3 \cdot 25 *\end{array}$ \\
\hline $\begin{array}{l}\mathrm{E} \text { (Similarity) } \\
\mathrm{AE} \\
\mathrm{BE} \\
\mathrm{CE} \\
\mathrm{ABE} \\
\mathrm{ACE} \\
\mathrm{BCE} \\
\mathrm{ABCE} \\
\mathrm{E} \mathrm{X} \text { Ss/Groups }\end{array}$ & $\begin{array}{r}1 \\
1 \\
1 \\
1 \\
1 \\
1 \\
1 \\
1 \\
16\end{array}$ & $\begin{array}{r}68.8802 \\
3.2552 \\
.4219 \\
4.3802 \\
4.3802 \\
4.3802 \\
.6302 \\
.1302 \\
1.5729\end{array}$ & $\begin{array}{c}43.79 * * * \\
2.07 \\
2.78 \\
2.78 \\
2.78\end{array}$ \\
\hline $\begin{array}{l}\mathrm{DE} \\
\mathrm{ADE} \\
\mathrm{BDE} \\
\mathrm{CDE} \\
\mathrm{ABDE} \\
\mathrm{ACDE} \\
\mathrm{BCDE} \\
\mathrm{ABCDE} \\
\mathrm{DE} \times \mathrm{Ss} / \text { Groups }\end{array}$ & $\begin{array}{r}3 \\
3 \\
3 \\
3 \\
3 \\
3 \\
3 \\
3 \\
48\end{array}$ & $\begin{array}{r}2.5469 \\
3.0608 \\
1.6163 \\
6.5469 \\
1.7115 \\
4.0120 \\
.4358 \\
.8615 \\
2.5729\end{array}$ & $\begin{array}{l}1.19 \\
2.54 \\
1.56\end{array}$ \\
\hline Total & 191 & & \\
\hline
\end{tabular}


REFERENCES

Anderson, N.S. Poststimulus cuing in immediate memory. J. exp. Psychol., 1960, 60, 216-221.

Archer, E.J. Identification of visual patterns as a function of information load. J. exp. Psychol., 1954, 48, $313-317$.

Archer, E.J. A re-evaluation of the meaningfulness of all possible CVC trigrams. Psychol. Monog., 1960, 74, 10 (497).

Archer, E.J. Concept identification as a function of obviousness of relevant and irrelevant information. J. exp. Psychol., 1962, 63, 616-620.

Averbach, E. (Visual masking). Unpublished experiments, Bell Telephone Laboratories, 1959 (cited in Sperling, 1960); cinema film (with Coriell), Bell Telephone Laboratories, no date, copy via N.L.Munn and J.E. Ritchie.

Averbach, E. \& Coriell, A.S. Short-term memory in vision. Bell Sys. tech. J., 1961, 40, 309-328. (Cited in Eriksen \& Hoffman, 1963).

Baldwin, R.D., Wright, A.D. \& Lehr, D.J. Relation between radar detection and the observer's concept of a target. J. appl. Psychol., 1964, 48, 81-83.

Bartlett, F.C. An experimental study of some problems of perceiving and imaging. Brit. J. Psychol., 1916, $8,222-266$.

Boring, E.G. Attribute and sensation. Am. J. Psychol., 1924, $35,301-304$.

Bricker, P.D. \& Chapanis, A. Do incorrectly perceived tachistoscopic stimuli convey some information? Psych. Rev., 1953, 60, 181-188. 
Broadbent, D.E. Immediate memory and simultaneous stimuli. Quart. J. exp. Psychol., 1957, 9, 1-11.

Broadbent, D.E. Perception and Communication. London: Pergamon, 1958.

Brown, J. The nature of set-to-learn and of intra-material interference in immediate memory. Quart. J. exp. Psychol., 1954, 6, 141-148.

Brown, J. Evidence for a selective process during perception of tachistoscopically presented stimuli. J. exp. Psychol., 1960, 59, 176-181.

Brown, J. Two tests of all-or-none learning and retention. Quart. J. exp. Psychol., 1964, 16, 123-133.

Cattell, R.B. A culture-free test. New York: The Psychological Corporation, 1944.

Carmichael, L., Hogan, H.P. \& Wlater, A.A. An experimental study of the effect of language on the reproduction of visually perceived form. J. exp. Psychol., 1932, 15, 73-86.

Chapanis, A. An exact multinomial one-sample test of significance. Psych. Bull., 1962, 59, 306-310; 532.

Chapman, D.W. Relative effects of determinate and indeterminate Aufgaben. Am. J. Psychol., 1932, 44, 163-174.

Differential Aptitude Tests (DAT) (Bennett, G.K., Seashore, H.G. \& Wesman, A.G.). New York: The Psychological Corporation, 1947.

Eckstrand, G.A. \& Wickens, D.D. Transfer of perceptual set. J. exp. Psychol., 1954, 47, 274-278.

Eriksen, C.W. \& Hoffman, M. Form recognition at brief durations as a function of adapting field and interval between stimulations. J. exp. Psychol., $1963,66,485-499$. 
Freeman, J.T. \& Engler, J. Perceptual recognition thresholds as a function of multiple and single set and frequency of usage of the stimulus material. Percept. Mot. Skills, 1955, 5, 149-154.

Foster, H. The operation of set in a visual search task. J. exp. Psychol., 1962, 63, 74-83.

Garner, W.R. Uncertainty and Structure as Psychological Concepts. New York: Wiley, 1962.

Goldiamond, I. Perception. Report ESD TR61-21 (1961), prepublication circulation of chapter in Bachrach, A.J. (Ed.): Experimental Foundations of Clinical Psychology (New York: Basic Books, 1962).

Gridgeman, N.T. Exact multinomial tests: Note on a paper by Alphonse Chapanis. Psych. Bull., 1964, 61, 239-240.

Green, B.F. \& Anderson, L.K. Colour coding in a visual search task. J. exp. Psychol., 1956, 51, 19-24.

Grunbaum, A.A. Ueber die Abstraktion der Gleichheit. Archiv fur die ges. Psychol., 1908, 12, 340-478.

Haber, R.N. A replication of selective attention and coding in visual perception. J. exp. Psychol., 1964, 67, 402-404.

Harcum, E.R., Filion, R.D.L. \& Dyer, D.W. Distribution of errors in tachistoscopic reproduction of binary patterns after practice. Percept. Mot. Skills, $1962,15,83-89$.

Harlow, H.F. The formation of learning sets. Psych. Rev., $1949,56,51-65$.

Harris, C.S. \& Haber, R.N. Selective attention and coding in visual perception. J. exp. Psychol., 1963, 65, $328-333$.

Hodge, M.H. The influence of irrelevant information upon complex visual discrimination. J. exp. Psychol., $1959,57,1-5$. 
Humphrey, G. Thinking: an introduction to its experimental psycholnov. Tinninn. Mathuan 1051 
Long, E.R., Henneman, R.H. \& Reid, L.S. An experimental analysis of set: Variables influencing the identification of ambiguous, visual stimulus objects. Am. J. Psych., 1960, 73, 533-562.

Long, E.R., \& Lee, W.A. The influence of specific stimulus cuing on location responses. USAF WADC tech. rep., Dec. 1953, 53-314.

McGinnies, E. Emotionality and perceptual defence. Psych. Rev., 1949, 56, 244-251.

McNemar, Q. Psychological Statistics (3rd ed.). New York: Wiley, 1962.

Marx, M.H., Murphy, W.W. \& Brownstein, A.J. Recognition of complex visual stimuli as a function of training with abstracted patterns. J.exp. Psychol., 1961, $62,456-460$.

Miller, G.A. The magic number seven, plus or minus two: Some limits on our capacity for processing information. Psych. Rev., 1956, 63, 81-97.

Moore, T.V. The process of recognition. Atti del V. Congresso internazionale di Psicologia tenuto in Roma, dal 26 al 30 Aprile, 1905, 286-287. (Author's trans. in Moore, 1910, 113-115).

Moore, T.V. The process of abstraction. Univ. of Calif. Publ. in Psych., 1910, 1, 73-197 (2).

Murdock, B.B., Jr. Perceptual defence and threshold measurements. J. Pers., 1954, 22, 565-571.

Murdock, B.B., Jr. Short-term retention of single paired associates. J. exp. Psychol., 1963, 65, 433-443.

Noble, C.E. Measurement of association value (a), rated associations $\left(\mathrm{a}^{\prime}\right)$, and scaled meaningfulness ( $\left.\mathrm{m}^{\prime}\right)$ for the 2100 CVC combinations of the English alphabet. Psych. Rep., 1961, 8, 487-521. 
Otis, A.S. Group intelligence scale. London: Harrap, 1937. Pearson, E.S. \& Hartley, H.O. (Eds.). Biometrika Tables for Statisticians. London, 1958.

Penrose, L.S. Pattern perception test. Melbourne: McCarron, Bird, 1947.

Pollack, I. Message uncertainty and message reception. J. acoust. soc. Amer., 1959, 31, 1500-1508.

Postman, I. Perception and learning. In S. Koch, Ed.: Psychology: A study of a science, vol.5, 30-113. New York: McGaw-Hill, 1963.

Postman, 1., \& Bruner, J.S. Multiplicity of set as a determinant of perceptual behaviour. J. exp. Psychol., 1949, 39, 369-377.

Reid, L.S., Henneman, R.H. \& Long, E.R. An experimental analysis of set: The effect of categorical restriction. Am. J. Psychol., 1960, 73, 568-572.

Rubin, E. Bericht uber experimentella Untersuchungen der Abstraktion. Z. f. Psychol., 1913, 63, 386-397.

Schiller, P.H. \& Wiener, M. Monoptic and dichoptic visual masking. J. exp. Psychol., 1963, 66, 386-393.

Schoenfeld, W.N. \& Cumming, W.W. Behaviour and perception. In S. Koch, Ed.: Psychology: A study of a science, vol. 5, 213-252. New York: McGraw-Hill, 1963.

Siipola, E.M. A group study of some effects of preparatory sets. Psychol. Monog., 1935, 46 (210).

Sperling, G. Information available in a brief visual presentation. Unpublished doctor's dissertation, Department of Psychology, Harvard University, 1959.

Sperling, G. The information available in brief visual presentations. Psychol. Monog., 1960, 74, 11 (498).

Swets, J.A., Tanner, W.P., Jr., \& Birdsall, T.G. Decision processes in perception. Psychol. Rev., 1961, 68, 301-340. 
Tanner, W.P., Jr. On the design of psychophysical experiments. In Quastler, H., Ed.: Information Theory in Psychology, 403-414. Glencoe: Free Press, 1955.

Titchener, E.B. Sensation and system. Am. J. Psychol., 1915, $26,258-267$.

Thorndike, E.I. \& Lorge, I. The teacher's word book of 30,000 words. New York: Columbia Univ., 1944.

Werner, H. Studies on contour: I. Qualitative analysis. Am. J. Psychol., 1935, 47, 40-64.

Wilcocks, R.W. An examination of Kulpe's experiments on abstraction. Am. J. Psych., 1925, 36, 324-341.

Winer, B.J. Statistical principles in experimental design. New York: McGraw-Hill, 1962.

Woodworth, R.S. Experimental psychology. New York: 1938. 